\title{
ASSOCIATIONS BETWEEN POSTTRAUMATIC STRESS DISORDER AND SUBSTANCE USE: A LONGITUDINAL INVESTIGATION OF INDIVIDUALS RECENTLY EXPOSED TO TRAUMA
}

by

\author{
Sonya G. Wanklyn \\ Master of Arts, Ryerson University, October 2011 \\ Bachelor of Arts, York University, June 2008
}

A dissertation

presented to Ryerson University

in partial fulfillment of the

requirements for the degree of

Doctor of Philosophy

in the Program of Psychology

Toronto, Ontario, Canada, 2016

(C) (Sonya G. Wanklyn) 2016 


\section{Author's Declaration for Electronic Submission of a Dissertation}

I hereby declare that I am the sole author of this dissertation. This is a true copy of the dissertation, including any required final revisions, as accepted by my examiners.

I authorize Ryerson University to lend this dissertation to other institutions or individuals for the purpose of scholarly research.

I further authorize Ryerson University to reproduce this dissertation by photocopying or by other means, in total or in part, at the request of other institutions or individuals for the purpose of scholarly research.

I understand that my dissertation may be made electronically available to the public. 
Associations between Posttraumatic Stress Disorder and Substance Use:

A Longitudinal Investigation of Individuals Recently Exposed to Trauma

Doctor of Philosophy 2016

Sonya G. Wanklyn

Psychology

Ryerson University

\begin{abstract}
Posttraumatic stress disorder (PTSD) and substance use disorder (SUD) commonly cooccur following trauma, and their co-occurrence is associated with substantial costs; however, our understanding of the timing and sequencing of these posttrauma mental health conditions is limited. This study examined the trajectories of PTSD symptom severity and substance use among individuals recently exposed to a traumatic event, with a focus on the potential moderating roles of PTSD and SUD diagnoses at the final assessment. Additionally, in attempt to better understand the functional relationship between PTSD symptoms and substance use posttrauma, this study compared models reflecting the theories of self-medication, susceptibility, and mutual maintenance. Participants included 137 individuals who had experienced a traumatic event within 6 months prior to study enrollment. Participants completed four assessments over an approximate 1-year period that included clinician-administered measures for DSM-5 PTSD symptoms and SUD diagnosis and self-report measures of alcohol and drug use. Change over time in PTSD symptoms and substance use by diagnostic status were investigated using growth curve models. Temporal sequencing between PTSD symptom severity and substance use was investigated with bivariate latent difference score structural equation modeling. In line with the conceptualization of PTSD as a disorder of impeded recovery, having a diagnosis of PTSD at the
\end{abstract}


final assessment moderated the trajectory of PTSD symptom severity such that symptom severity declined only among those without PTSD. In contrast, the influence of SUD appeared to be negligible. Both PTSD and SUD diagnoses were associated with initial drug use frequency. However, the relationship between alcohol use and diagnostic status did not reach statistical significance. Regarding temporal relationships between PTSD symptoms and substance use, significant and negative PTSD to change in substance use cross-lagged paths were found across most of the models, while only one significant substance use to change in PTSD severity crosslagged path emerged in the PTSD intrusion with alcohol use model. These results add to a growing body of research suggesting trauma-focused intervention is viable for individuals with PTSD/SUD. Further, examination of potential mediators and moderators of the relation between PTSD and SUD is recommended as a critical focus for future research. 


\section{Acknowledgements}

I would like to express my sincerest gratitude to my supervisor, Dr. Candice Monson, for her mentorship over the course of my doctoral degree. Her guidance and wisdom have been invaluable to my personal and professional growth, and I will no doubt continue to draw on the lessons that I have learned from her for many years to come. Dr. Martin Antony and Dr. Todd Girard also played a key role in this dissertation. A heartfelt thank you to Dr. Antony for his insightful feedback and constant support, and much appreciation to Dr. Girard for his analytic guidance and unwavering excitement for all things statistical. Finally, I would like to thank Dr. Ruth Lanius and Dr. Elizabeth McKay and for their gift of time as members of my examining committee. 


\section{Table of Contents}

Author's Declaration for Electronic Submission of a Dissertation ............................................ ii

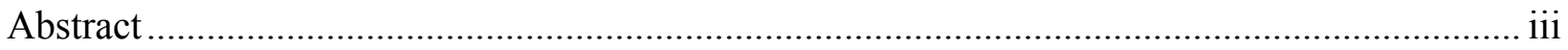

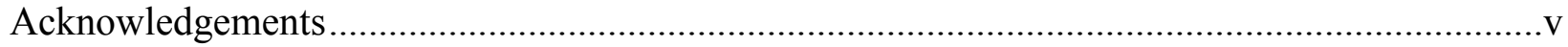

Table of Contents ......................................................................................................... vi

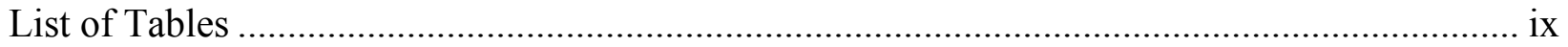

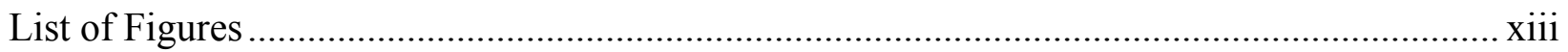

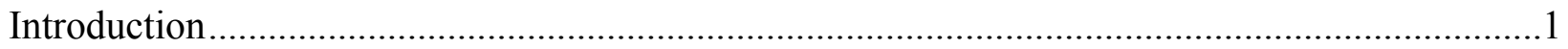

Co-occurrence and Costs of Posttraumatic Stress Disorder and Substance Use Disorders

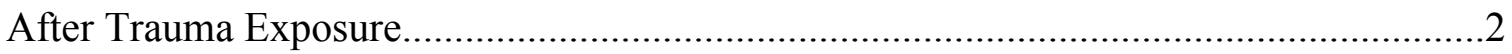

Trajectory of PTSD Symptom Severity and Substance Use Following Trauma

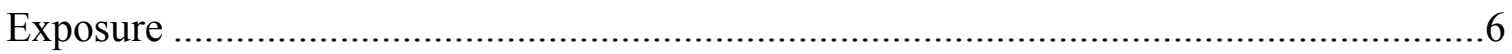

Theories Accounting for the Association Between PTSD Symptoms and Substance Use

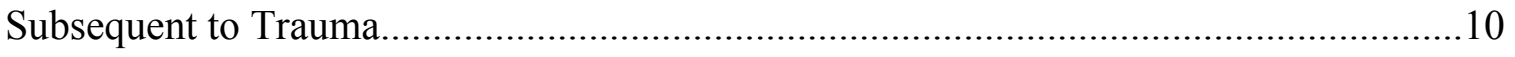

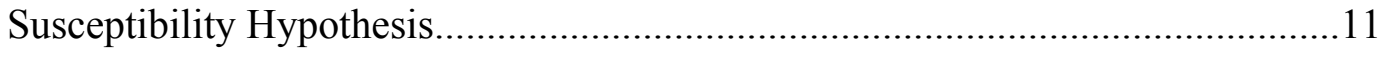

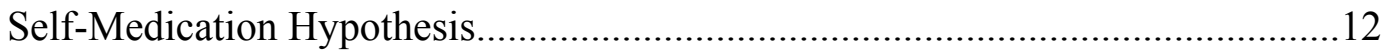

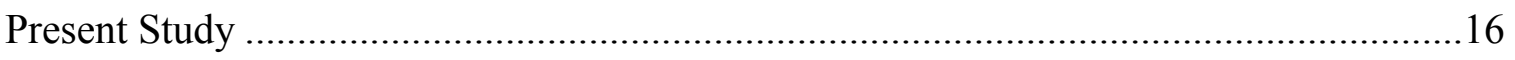

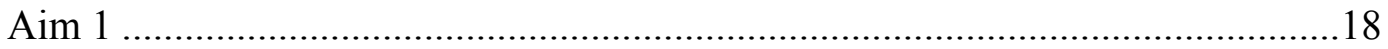

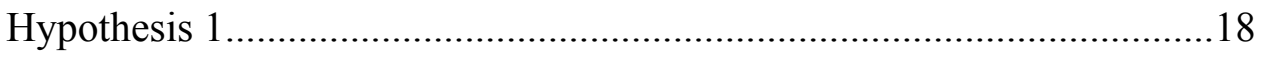

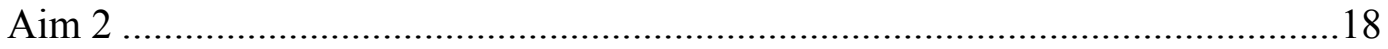

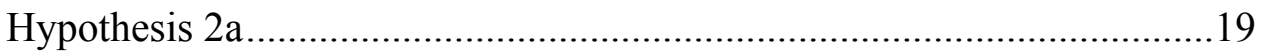

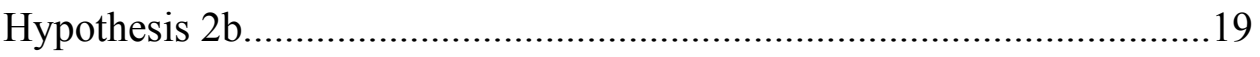

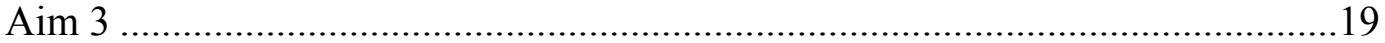


Hypothesis 3a.

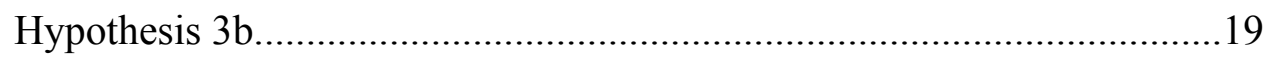

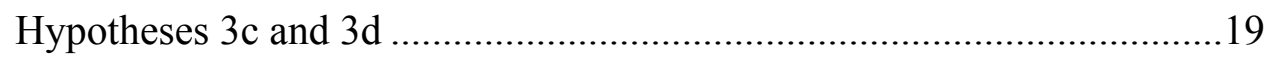

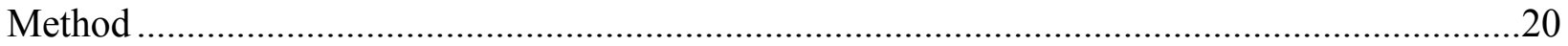

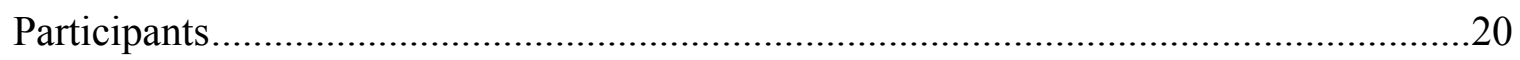

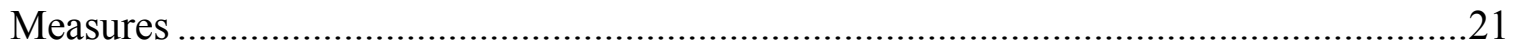

Clinician-Administered PTSD Scale _.............................................................21

MINI International Neuropsychiatric Interview ………................................26

Frequency of Drug and Alcohol Use ...............................................................27

Mental Health Utilization Questionnaire ........................................................28

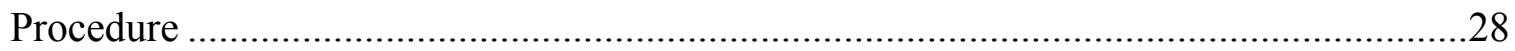

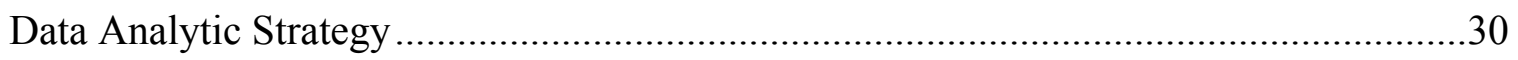

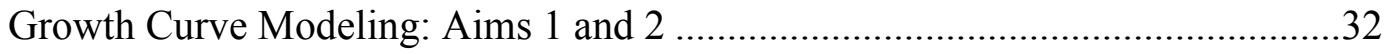

Bivariate Latent Difference Score Structural Equation Modeling: Aim 3 .............36

Univariate LDS Models for Outcome Variables ..........................................39

Measurement Model and Univariate LDS models for PTSD Total

Symptom Severity: Testing Hypothesis 3a ..............................................46

Constructing and Testing BLDS Models Using Best-fit Univariate LDS

Models for Each Outcome Variable: Testing Hypotheses 3b

Through $3 \mathrm{~d}$

BLDS Models for PTSD and Substance Use: Hypothesis $3 \mathrm{~b}$ .53

Self-Medication Versus Susceptibility Theory Models: Hypothesis 3c ...53 
Self-Medication Versus Mutual Maintenance Theory Models:

Hypothesis $3 \mathrm{~d}$

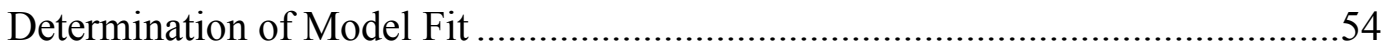

Results.

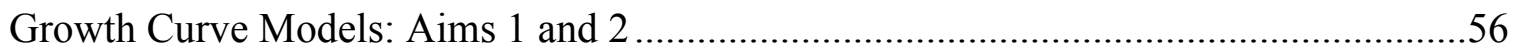

Bivariate Latent Difference Score Structural Equation Models: Aim 3 .........................66

Univariate LDS Models for Outcome Variables ............................................69

BLDS Models for PTSD and Substance Use..............................................79

Self-Medication Versus Susceptibility Models: Hypothesis 3c..............112

Self-Medication Versus Mutual Maintenance Theory Models:

Hypothesis $3 \mathrm{~d}$

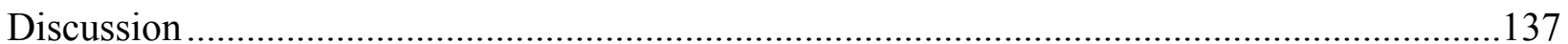

Aim 1: Trajectory of PTSD Symptom Severity Posttrauma.......................................143

Aim 2: Trajectories of Alcohol and Drug Use Posttrauma........................................147

Aim 3: Temporal Sequencing of PTSD Symptom Severity and Substance Use

Posttrauma.

Limitations and Future Directions ................................................................... 158

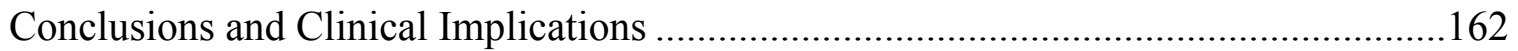

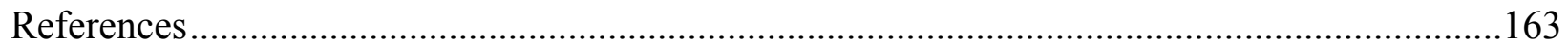




\section{List of Tables}

Table 1: Demographic Information for the Sample at Initial Assessment

Table 2: Prevalence of DSM-5 Current and Lifetime Mental Health Conditions for the Sample at Initial Assessment and Type of Trauma Exposure Associated with Study Enrolment

Table 3: Skew and Kurtosis for Original and Transformed Variables Used in Growth Curve Models Related to Aims 1 and 2

Table 4: Bivariate Latent Difference Score Structural Equation Model Parameters

Table 5: Means and Standard Deviations for Variables of Interest Across Each of the Four Assessment Time Points

Table 6: Pearson Correlation Matrix for Variables at Each Assessment Point for the Total Sample

Table 7: Growth Curve Models Predicting Initial PTSD Total Symptom Severity and Symptom Trajectory from End-State Diagnoses

Table 8: Growth Curve Models Predicting Initial Alcohol Use Frequency and Trajectory of Alcohol Use from End-State Diagnoses

Table 9: Growth Curve Models Predicting Initial Drug Use Frequency and Trajectory of Drug Use from End-State Diagnoses .67

Table 10: Means and Standard Deviations (Based on Days Since Traumatic Event Exposure) for

Time Structures 68

Table 11: Deviance Statistics and Parameter Estimates for the Three Growth Curve Models Varied by Time Representation. 
Table 12: Goodness of Fit Estimates for Univariate Latent Difference Score Models for PTSD Symptom Clusters

Table 13: Parameter Estimates for Best-Fitting Univariate Latent Difference Score Models for PTSD Symptom Clusters .75

Table 14: Goodness of Fit Estimates for Univariate Latent Difference Score Models for Substance Use Frequencies .77

Table 15: Parameter Estimates for Best-Fitting Univariate Latent Difference Score Models for Substance Use Frequencies .80

Table 16: Goodness of Fit Estimates for 4-Indicator and Single-indicator Latent Difference Score Models for PTSD Total Severity.

Table 17: Parameter Estimates for Best-Fitting Univariate Latent Difference Score Model for PTSD Total Symptom Severity

Table 18: Goodness of Fit Estimates for Bivariate Latent Difference Score Models .87

Table 19: Bivariate Latent Difference Score Model for PTSD Total Symptom Severity with Alcohol Use Frequency

Table 20: Bivariate Latent Difference Score Model for PTSD Intrusion Cluster Severity with Alcohol Use Frequency

Table 21: Bivariate Latent Difference Score Model for PTSD Hyperarousal Cluster Severity with Alcohol Use Frequency .93

Table 22: Bivariate Latent Difference Score Model for PTSD Avoidance Cluster Severity with Alcohol Use Frequency .95

Table 23: Bivariate Latent Difference Score Model for PTSD Negative Shifts in Cognitions and Mood Cluster Severity with Alcohol Use Frequency .97 
Table 24: Bivariate Latent Difference Score Model for PTSD Total Symptom Severity with Drug Use Frequency

Table 25: Bivariate Latent Difference Score Model for PTSD Intrusion Cluster Severity with Drug Use Frequency. 104

Table 26: Bivariate Latent Difference Score Model for PTSD Hyperarousal Cluster Severity with Drug Use Frequency. .106

Table 27: Bivariate Latent Difference Score Model for PTSD Avoidance Cluster Severity with Drug Use Frequency. .108

Table 28: Bivariate Latent Difference Score Model for PTSD Negative Shifts in Cognitions and Mood Cluster Severity with Drug Use Frequency .....

Table 29: Goodness of Fit Estimates for Self-Medication and Susceptibility Theory Bivariate Latent Difference Score Models

Table 30: Self-Medication Theory Bivariate Latent Difference Score Model for PTSD Total Symptom Severity with Alcohol Use Frequency

Table 31: Self-Medication Theory Bivariate Latent Difference Score Model for PTSD Hyperarousal Cluster Severity with Alcohol Use Frequency 118

Table 32: Self-Medication Theory Bivariate Latent Difference Score Model for PTSD Avoidance Cluster Severity with Alcohol Use Frequency. 120

Table 33: Self-Medication Theory Bivariate Latent Difference Score Model for PTSD Negative Shifts in Cognitions and Mood Cluster Severity with Alcohol Use Frequency. 122

Table 34: Susceptibility Theory Bivariate Latent Difference Score Model for PTSD Intrusion Cluster Severity with Alcohol Use Frequency 
Table 35: Self-Medication Theory Bivariate Latent Difference Score Model for PTSD Total Symptom Severity with Drug Use Frequency

Table 36: Self-Medication Theory Bivariate Latent Difference Score Model for PTSD Intrusion

Cluster Severity with Drug Use Frequency.

Table 37: Self-Medication Theory Bivariate Latent Difference Score Model for PTSD

Hyperarousal Cluster Severity with Drug Use Frequency

Table 38: Self-Medication Theory Bivariate Latent Difference Score Model for PTSD

Avoidance Cluster Severity with Drug Use Frequency

Table 39: Self-Medication Theory Bivariate Latent Difference Score Model for PTSD Negative Shifts in Cognitions and Mood Cluster Severity with Drug Use Frequency

Table 40: Self-Medication Theory Bivariate Latent Difference Score Model for PTSD Intrusion Cluster with Alcohol Use Frequency 138

Table 41: Goodness of Fit Estimates for Self-Medication and Mutual Maintenance Theory Bivariate Latent Difference Score Models 140 


\section{List of Figures}

Figure 1: Consort Diagram Depicting Participant Flow Through the Study

Figure 2: Hierarchical Four-Indicator Measurement Model of PTSD Symptom Severity, with Indicators Based on DSM-5 PTSD Symptom Clusters.

Figure 3: Path Diagrams For the Six Univariate Latent Difference Score Models Based on the 120-Day Time Structure Using PTSD as the Construct of Interest

Figure 4: Example Bivariate Latent Difference Score Model With Four Time Points .48

Figure 5: Trajectories of PTSD Total Symptom Severity, Alcohol Use Frequency, and Drug Use Frequency Based on End-State PTSD and/or SUD Diagnostic Status .63

Figure 6: Bivariate Latent Difference Score Model Examining Associations Between PTSD and Alcohol Use Subsequent to Traumatic Exposure .84

Figure 7: Bivariate Latent Difference Score Model Examining Associations Between PTSD and Drug Use Subsequent to Traumatic Exposure .100 


\section{Associations between Posttraumatic Stress Disorder and Substance Use:}

\section{A Longitudinal Investigation of Individuals Recently Exposed to Trauma}

Over $75 \%$ of Canadians are exposed to at least one traumatic event during their lives, 9.2\% of whom develop posttraumatic stress disorder (PTSD; Van Ameringen, Mancini, Patterson, \& Boyle, 2008). Problematic substance use and substance use disorder (SUD) are also common following traumatization (e.g., DiMaggio, Galea, \& Guohua, 2009), whereby both PTSD and SUD are pernicious and debilitating mental health conditions that are associated with substantial personal and societal costs (Kessler, Sonnega, Bromet, Huges, \& Nelson, 1995; Rehm et al., 2006; Zatzick et al., 1997). Furthermore, the complex presentation of co-occurring PTSD/SUD has serious financial, health, and treatment-related implications beyond those associated with either disorder alone (e.g., Blanco et al., 2013). For instance, unlike other disorders that commonly co-occur with PTSD such as major depressive disorder (Powers, Halpern, Ferenschak, Gillihan, \& Foa, 2010), SUD does not resolve with trauma-focused PTSD treatments. Also, the presence of SUD is often considered a contraindication for PTSD treatment, and randomized controlled trials of PTSD typically exclude individuals with co-occurring SUD (e.g., Monson et al., 2006). With respect to SUD treatment, individuals with PTSD typically have poorer treatment outcomes (Norman, Tate, Anderson, \& Brown, 2007). Although promising findings have been documented for trauma-focused psychotherapies targeting this comorbidity (e.g., Mills et al., 2012), currently available treatments do not appear to result in better SUD-related outcomes compared to SUD treatment as usual (Schumm \& Gore, 2016). Yet each of these disorders is generally investigated without consideration of their likely interplay.

Understanding of the timing and sequencing of these posttrauma mental health problems is still in its infancy. Although the course of PTSD is relatively well documented, less is known 
about changes in PTSD symptom severity in relation to co-occurring SUD. Similarly, the course of substance use following traumatic exposure is rarely investigated. Moreover, few attempts have been made to understand the dynamic influence of PTSD symptoms on substance use, and vice versa, subsequent to trauma. The majority of studies have been cross-sectional and descriptive only, or focus exclusively on individuals with long-standing PTSD. For these reasons, it is unclear whether PTSD is a determinant of SUD or vice versa. The direction of this relationship is critical to informing the development of the most efficacious and parsimonious prevention and intervention efforts for the majority of Canadians who will be exposed to trauma. Thus, the purpose of this prospective longitudinal study was to address this gap in the literature by examining the trajectories of PTSD symptom severity and substance use subsequent to traumatic exposure based on PTSD and/or SUD diagnostic status and the temporal relationships between PTSD symptom severity and substance use posttrauma.

\section{Co-occurrence and Costs of Posttraumatic Stress Disorder and Substance Use Disorders}

\section{After Trauma Exposure}

PTSD is a condition that may result following traumatic event exposure. According to the Diagnostic and Statistical Manual of Mental Disorders, Fifth Edition (DSM-5; American Psychiatric Association [APA], 2013), traumatic events are defined (in Criterion A) as being exposed to an event involving actual or threatened death, serious injury, or sexual violence by direct exposure, witnessing (in person), or experiencing indirectly by learning that a close other was exposed to the trauma. Criterion A events can also involve extreme or repeated indirect exposure to details of traumatic events that are aversive, typically occurring in the course of professional service (e.g., first responders). PTSD is characterized by persistence of intrusion symptoms (e.g., recurrent intrusive memories, trauma-related nightmares), efforts to avoid 
trauma stimuli, traumatic event-related negative shifts in cognitions or mood (e.g., restricted range of affect, feeling detached from others, distorted negative beliefs and expectations), and increased arousal and reactivity associated with the trauma (e.g., sleep disturbance, hypervigilance, irritable or aggressive behaviour). These symptoms must be present for at least 1 month and result in clinically significant distress to be diagnosed with the disorder (APA, 2013).

The costs associated with PTSD are substantial. Extending well beyond the impact of these symptoms alone, research has consistently documented strong relationships between PTSD and health and functional impairments. For instance, individuals with PTSD have increased occupational impairment, higher rates of absenteeism, and are at elevated risk of unemployment compared to individuals without PTSD (Hoge, Terhakopian, Castro, Messer, \& Engel, 2007; Magruder et al., 2004; Resnick \& Rosenheck, 2008). In addition, individuals with PTSD incur higher healthcare costs, experience increased medical morbidity, are at increased risk for suicide attempts, and use more medical services than individuals with other mental health conditions (Boscarino, 2006; Cohen et al., 2010; Kessler, 2000; Schnurr \& Green, 2004; Vasterling et al., 2008; Walker et al., 2003); associations that have been demonstrated to persist even after controlling for physical injury (Hoge et al., 2007). Taken together, PTSD is associated with enormous societal and health-related costs, contributing to an economic burden of at least $\$ 40$ billion per year in the United States (Kessler, 2000). Unlike some mental health diagnoses that tend to wax and wane over time, PTSD, once developed, generally has a chronic and pernicious course (e.g., Bremner, Southwick, Darnell, \& Charney, 1996). Furthermore, PTSD is frequently associated with other mental health disorders; one of the most common is SUD (e.g., Kessler et al., 1995; Van Ameringen et al., 2008). 
SUDs are also common after trauma. As defined by the DSM-5 (APA, 2013), SUDs are grouped by drug class and are characterized by a maladaptive pattern of use leading to clinical distress or impairment. SUD is manifested by recurrence of at least two of the following within a 12-month period: (a) tolerance of the substance; (b) withdrawal symptoms subsequent to substance use; (c) using more of the substance than intended or over a longer period than planned; (d) unsuccessful efforts or persistent desire to reduce or cease using the substance; (e) significant time spent using, obtaining, or recovering from the effects of the substance; (f) strong desire or urge to use the substance; (g) failure to fulfill major obligations at work, school, or home due to substance use; (h) continued use despite interpersonal problems likely exacerbated or caused by the substance; (i) reduced social, recreational, or occupational activities due to substance use; (j) substance use in physically hazardous situations; and (k) continued use despite physical or psychological problems likely exacerbated or caused by the substance (APA, 2013).

The social, economic, morbidity, and mortality costs attributable to SUDs are extensive. The annual cost of substance dependence among Canadians related to alcohol and illicit drug use has been estimated at 22.8 billion, involving productivity losses, direct health care costs, and law enforcement expenses, among others (Rehm et al., 2006). Research has demonstrated that the relative risk for several types of cancers, epilepsy, liver cirrhosis, and cardiovascular disease increase with heavy alcohol consumption (Aronson, 2003; Bagnardi et al., 2001; Mann, Smart, \& Govoni, 2003; Rehm, Gmel, Sempos, \& Trevisan, 2003; Rodés, Salaspuro, \& Sorensen, 1999). SUD and problematic substance use have also been identified as robust risk factors for intimate partner violence (Afifi, Henriksen, Asmundson, \& Sareen, 2012; Foran \& O’Leary, 2008; Stith, Smith, Penn, Ward, \& Tritt, 2004) and are associated with central nervous system changes and cognitive deficits (e.g., Bates, Bowden, \& Barry, 2002; Jernigan \& Ostergaard, 1995; Parsons, 
1998). In addition, individuals with SUD are at increased risk for attempted suicide compared to those without SUD (e.g., Piselli, Elisei, Murgia, Quartesan, \& Abram, 2009).

Research consistently documents high rates of co-occurrence between PTSD and SUD. Men and women with PTSD have been identified as 51.9\% and 27.9\% more likely, respectively, to have a SUD compared to individuals with no PTSD diagnosis (Brady, Back, \& Coffey, 2004). In addition, population-based surveys have identified individuals with PTSD to be at a substantially higher likelihood for subsequent onset of a SUD (odds ratios between 2.0 and 4.2) than individuals without PTSD (Kessler, 2000), while persons with SUD are up to ten times more likely to have PTSD compared to their non-SUD counterparts (Chilcoat \& Menard, 2003; Kessler et al., 1995). In addition, as many as $40 \%$ of individuals seeking treatment for SUD have current PTSD (Driessen et al., 2008; Jacobsen, Southwick, \& Kosten, 2001; Ouimette, Read, \& Brown, 2005; Read, Brown, \& Kahler, 2004).

With respect to costs, PTSD/SUD comorbidity is related to a worse clinical profile than either disorder alone. For instance, PTSD/SUD is associated with more severe PTSD symptoms than a diagnosis of PTSD-only, particularly symptoms relating to avoidance and hyperarousal (Blanco et al., 2013; Saladin, Brady, Dansky, \& Kilpatrick, 1995). The comorbidity is also associated with increased severity of SUD symptomatology as compared to a diagnosis of SUDonly (Driessen et al., 2008). In addition, PTSD/SUD is related to an increased likelihood of attempted suicide and higher rates of other mental health problems than either disorder alone (Back et al., 2000; Blanco et al., 2013), as well as lower socioeconomic status and higher rates of unemployment (Riggs, Rukstalis, Volpicelli, Kalmanson, \& Foa, 2003). PTSD/SUD, when compared to SUD-only, is associated with more neurological, cardiovascular, and chronic medical symptoms (Ouimette, Goodwin, \& Brown, 2006), as well as higher rates of chronic 
health problems (Tate, Norman, McQuaid, \& Brown, 2007). Furthermore, treatment outcome research indicates that individuals with PTSD/SUD have higher impairment throughout treatment (Najavits et al., 2007), poorer substance use outcomes, and higher rates of substance use relapse (Norman et al., 2007) relative to those with SUD-only (Ouimette, Brown, Najavits, 1998).

In summary, the costs of PTSD and SUD are extensive, and research suggests their cooccurrence renders individuals more vulnerable to a host of poorer outcomes as compared to either diagnosis in isolation. Furthermore, high rates of PTSD and SUD co-occurrence suggest that these disorders may be functionally related. Also, sequential treatments do not appear to be efficacious in the treatment of individuals with this comorbidity, and no treatment targeting PTSD/SUD has yet been demonstrated as superior to SUD treatment as usual for reducing SUDrelated outcomes (Schumm \& Gore, 2016). Thus, attention to this relationship is warranted and is best investigated longitudinally subsequent to traumatic event exposure.

\section{Trajectory of PTSD Symptom Severity and Substance Use Following Trauma Exposure}

PTSD is often conceptualized as a disorder of impeded recovery in that most individuals experience symptoms similar to those of PTSD in the immediate aftermath of traumatization, but these symptoms abate for the majority of individuals without intervention during the initial months after exposure (Foa \& Rothbaum, 1998; Monson \& Fredman, 2012; Riggs, Rothbaum, \& Foa, 1995; Rothbaum, Foa, Riggs, Murdock, \& Walsh, 1992). For a minority of individuals, symptoms will persist over time and ultimately result in a diagnosis of PTSD (Kessler et al., 1995; Rothbaum et al., 1992; Van Ameringen et al., 2008). According to this conceptualization, factors may hinder natural recovery of symptoms posttrauma, thereby contributing to the onset of PTSD. 
Several prospective studies suggest that problematic substance use is associated with reduced natural recovery and increase risk for chronicity and severity of PTSD symptoms (e.g., Acierno, Resnick, Kilpatrick, Sauders, \& Best, 1999; Matar, Zohar, Kaplan, \& Cohen, 2009). Kaysen and colleagues (2006) examined the role of a prior diagnosis of alcohol use disorder (AUD) and alcohol-related problems (i.e., endorsement of at least one AUD symptom) on PTSD symptom severity subsequent to trauma in a community sample of female victims of sexual or physical assault. The women were assessed initially between 2 to 4 weeks postassault and again 3 months after the trauma. Results indicated that women with alcohol problems and AUD had significantly less PTSD symptom improvement at 3-months postassault. Similar results were found among individuals hospitalized for traumatic injuries (McFarlane et al., 2009), where problematic drinking and use indicative of alcohol dependence (as assessed by the AUDIT, Babor et al., 1989) within the 12 months prior to traumatic injury predicted PTSD symptom severity at the time of initial assessment (average of 7 days after injury). PTSD symptom severity was also higher at the 3-month follow-up assessment for those who endorsed alcohol dependence and related problems in the 3-month period following traumatization, as compared to those who did not endorse such items (McFarlane et al., 2009).

Although Kaysen et al. (2006) and McFarlane et al. (2009) employed longitudinal designs, they investigated acute responses within 3 months following trauma and over only two assessment points. In recognition of these limitations, Kaysen and colleagues (2011) examined the moderating effect of alcohol use and AUD on the course of PTSD over 6 months following traumatic exposure. Female sexual assault victims completed assessments within 5 weeks of their trauma, and at 3- and 6-months postassault. Using hierarchical linear modeling (HLM), they found less decline in PTSD symptoms over time for women who reported AUD at the time of 
assault compared to women without AUD. Peak drinking, days drinking, and overall amount of alcohol consumed within the month prior to assault were also examined, but none of these indices of alcohol consumption were found to moderate the course of PTSD symptoms.

Zatzick and colleagues (2002) prospectively investigated predictors of PTSD symptom severity in surgical inpatients between the ages of 14 and 65 years who had been hospitalized as a result of violent assault or motor vehicle accidents. The participants completed an initial assessment while in hospital, and then subsequently at 1-, 4-, and 12-months postinjury. A variety of factors (e.g., depression, chronic medical conditions, social support, severity of injury, demographic variables), as well as self-reported substance use in the month prior to injury and at time of admission via blood alcohol and urine drug toxicology, were assessed at baseline. PTSD symptoms at initial assessment and trauma exposure prior to the trauma leading to hospitalization were the strongest predictors of subsequent PTSD severity, followed by positive toxicology screen for stimulants at admission and female sex. Similar to Kaysen and colleagues (2011), alcohol consumption in the month prior to traumatization was not predictive of greater PTSD symptom severity over the course of the subsequent year.

Fewer studies have been conducted on the trajectory of substance use subsequent to traumatization, and the majority have been cross-sectional. Despite some studies demonstrating no significant changes in substance use (e.g., Nordløkken, Pape, Wentzel-Larsen, \& Heir, 2013) or decreases in SUDs or substance use after trauma (e.g., Gould, Ponsford, Johnston, \& Schönberger, 2011), the majority of research suggests that traumatic event exposure is related to increases in substance use. A meta-analytic review of the literature pertaining to substance use following mass terrorist incidents, including 31 studies, concluded that population rates of substance use following exposure were higher than general populations rates under normal 
circumstances (DiMaggio et al., 2009). Higher rates of substance use following trauma have also been documented among pretrauma substance abusing samples (e.g., Dewart, Blanche, \& Schmeidler, 2006).

In an investigation of substance use following the September 11,2001, terrorist attacks, Vlahov and colleagues (2002) randomly sampled residents living proximal to the World Trade Centre 5 to 8 weeks after the attacks. Results indicated that over $40 \%$ of those who drank alcohol prior to the attacks increased their frequency of consumption after the attacks. Stein and colleagues (2004) also examined psychological reactions following the September 11, 2001, terrorist attacks among a nationally representative sample of adults in the United States. This study included two time points, one immediately following the attacks and the second approximately 2 months later. Thirty-two percent of participants with persistent distress 2 months following the attack reported using nonprescription medications, alcohol, or other drugs within the preceding month as a result of the attack. Those with persistent distress were more likely to endorse substance use compared to individuals without substantial distress, as well as compared to individuals with improved symptoms relative to the first assessment.

PTSD may influence the course of substance use after trauma. For instance, Nordløkken and colleagues (2013) conducted a retrospective investigation of perceived changes in alcohol use after tsunami exposure. Although trauma exposure severity was associated with selfdescribed changes in alcohol use, these results became nonsignificant after controlling for PTSD symptoms. Pfefferbaum and Doughty (2001) examined the relationship between PTSD symptoms and alcohol use 6 months after the 1995 Oklahoma City federal building bombing. Participants included a subset of individuals who endorsed alcohol use as part of a larger study of individuals seeking mental health treatment subsequent to the bombing. Forty percent of 
individuals endorsed increased alcohol consumption following the bombing. Moreover, PTSD significantly predicted increased consumption even after controlling for trauma-related variables (i.e., initial reaction, physical exposure, physical injury).

\section{Theories Accounting for the Association Between PTSD Symptoms and Substance Use}

\section{Subsequent to Trauma}

Although it is recognized that co-occurrence of disorders can result from shared risk factors that predispose individuals to develop both PTSD and SUD (Kraemer, Stice, Kazdin, Offord, \& Kupfer, 2001), it is generally agreed that PTSD and SUD are causally related (e.g., Back, Brady, Jaanimägi, \& Jackson, 2006a; Bolton, Cox, Clara, \& Sareen, 2006). Numerous theories have been proposed to explain the phenomenon of comorbid PTSD/SUD, principal among them the susceptibility (Brady et al., 2004; Chilcoat \& Breslau, 1998) and self-medication (Khantzian, 1985; Stewart, 1996) hypotheses. The high-risk hypothesis (Chilcoat \& Breslau, 1998) is an additional theory that has been proposed and may explain the co-occurrence of PTSD and SUD. This hypothesis argues that problematic substance use, and the high-risk behaviours associated with individuals with SUDs, increase risk for exposure to potentially traumatic events (Chilcoat \& Breslau, 1998). In this way, it is suggested that substance use indirectly increases the likelihood for co-occurring PTSD. This study focused on the self-medication and susceptibility hypotheses because they both postulate relationships between PTSD and SUD subsequent to traumatic exposure.

Susceptibility Hypothesis. According to the susceptibility hypothesis, preexisting substance use or substance use in the immediate wake of trauma promotes the onset and maintenance of PTSD symptoms, thereby compromising natural recovery from traumatic exposure (Brady et al., 2004). Substance use is postulated to interfere with posttraumatic 
recovery by hindering emotional processing of the trauma (Brady \& Sinha, 2005). Also, physiological arousal associated with withdrawal from substances overlaps extensively with, and can evoke or exacerbate, PTSD symptoms (Jacobsen et al., 2001; Stewart \& Conrod, 2003).

Empirical research demonstrating the onset of substance use problems preceding PTSD has been used in support of this hypothesis. Cottler, Compton, Mager, Spitznagel, and Janca (1992) investigated lifetime prevalence of PTSD and substance use using a general population survey of psychiatric illness. They found onset of substance use preceded that of PTSD symptoms among individuals who endorsed a history of alcohol or illicit drug use. In addition, the relationship between type of substance and PTSD was explored by classifying individuals within mutually exclusive substance use groups: cocaine/opiates (individuals who reported using cocaine, heroin, or other opioids, regardless of whether they endorsed other drug or alcohol use), pills/hallucinogens (individuals who reported amphetamine, barbiturate, hallucinogen, or tranquilizer use, whether or not they use alcohol or marijuana), marijuana (drug use restricted to cannabis, with or without alcohol), and heavy alcohol (substance use restricted to problematic alcohol use). Those in each of the substance use groups, except marijuana, were more likely to meet criteria for probable PTSD as compared to nonusers. Study results also suggested that risk for PTSD differed based on type of substance use. Specifically, female sex and use of cocaine/opiates predicted probable PTSD, while other substance use categories, age, race, personality, and depression were not significant predictors (Cottler et al., 1992).

Further, a recent prospective study examined temporal ordering of PTSD symptoms in relation to alcohol use over a 3-year period (12 assessment points) among college students (Read, Wardell, \& Colder, 2013). Analyses were conducted using an application of structural equation modeling (SEM) with cross-lagged panels, the state-trait-error model, with separate 
models run for frequency of alcohol use and alcohol problems. Although reciprocal relationships were found in the alcohol use model, with PTSD symptom severity and alcohol consumption each predicting the other across the 3-year period, in the alcohol problems model, only the unidirectional pathways from alcohol problems to PTSD symptoms were significant.

Support for the susceptibility hypothesis also comes from studies that report individuals with alcohol or drug problems at the time of trauma exposure to evidence less PTSD symptom improvement over time as compared to those without such problems (e.g., Kaysen et al., 2011), as well as studies that document decreases in PTSD symptom severity during and subsequent to drug and/or alcohol abstinence. Coffey, Schumacher, Brady, and Cotton (2007) investigated change in PTSD symptoms during acute and protracted abstinence among 162 trauma-exposed participants recruited from outpatient and inpatient substance abuse treatment facilities. All participants were withdrawing from cocaine, alcohol, or both alcohol and cocaine, and $28 \%$ of the sample met criteria for current PTSD. Latent growth modeling demonstrated declines in PTSD symptoms across the 28-day study period, regardless of substance of abuse or initial diagnosis of PTSD. These results suggest trauma-related symptoms may be associated with acute and protracted substance abstinence. It is notable that, despite a decrease in PTSD severity during the abstinence period, individuals who initially met criteria for PTSD still reported symptoms indicative of probable PTSD at the final assessment point (Coffey et al., 2007).

Self-Medication Hypothesis. In contrast to the susceptibility hypothesis, the selfmedication hypothesis proposes that psychological disorders confer risk for SUDs (Khantzian, 1985). Within this framework, individuals are posited to use alcohol and drugs to alleviate emotional distress and cope with posttrauma sequelae (Stewart, 1996). Indeed, individuals with PTSD report acute symptom relief from consuming central nervous system depressants such as 
alcohol and opioids (Bremner et al., 1996). Moreover, the anxiolytic properties of many substances of abuse have been well-established and provide a theoretical basis to support a pathway whereby substance use may mitigate PTSD symptoms (Armony \& LeDoux, 1997; Brady et al., 2004). Furthermore, research has identified coping-motivated substance use to predict substance-related problems, even when controlling for frequency of use (e.g., Merrill \& Read, 2010). In this way, self-medication of PTSD symptoms through substance use might reduce the severity of PTSD symptoms in the short-term but is argued to lead to the development of comorbid SUD in the longer-term.

The majority of published literature related to PTSD/SUD is consistent with the selfmedication hypothesis (Jacobsen et al., 2001). This support comes from several lines of research, including nationally representative and community-based studies reporting high rates of individuals with comorbid disorders endorsing self-medication behaviours (Leeies, Pagura, Sareen, \& Bolton, 2010; Ullman, Filipas, Townsend, \& Starzynski, 2006). Relatedly, Back and colleagues (2006a) investigated patients' perceptions of the functional relationship between PTSD and SUD in a sample of individuals with comorbid PTSD and cocaine dependence. Sixtyfour percent of individuals identified a reduction in cocaine use following improvement in PTSD symptoms, while $87 \%$ noted increased cocaine use as a result of PTSD symptom deterioration.

Additional support for the self-medication hypothesis comes from treatment outcome studies comparing treatment responders and nonresponders to integrated PTSD/SUD treatment. Back, Brady, Sonne, and Verduin (2006b) investigated the temporal association of improvements between alcohol consumption and PTSD symptoms among individuals enrolled in a 12-week medication (i.e., sertraline) trial for the treatment of PTSD/SUD. Results revealed fewer percent days drinking and average drinks per day among PTSD treatment responders compared to PTSD 
treatment nonresponders (defined as less than 30\% decrease in Clinician Administered PTSD Scale total score from baseline to final assessment). When considering PTSD symptom improvement as a function of alcohol treatment response (defined as $\geq 75 \%$ decrease in percent days drinking and average drinks per day), no statistical differences were found in PTSD symptoms improvement between responders and nonresponders. Furthermore, support for the self-medication hypothesis is found in studies documenting PTSD to temporally precede SUDs (Chilcoat \& Breslau, 1998). For instance, an investigation of SUDs in relation to mood and anxiety disorders revealed that baseline PTSD predicted later onset of SUD, while baseline SUD did not predict new onset PTSD (Wolitzky-Taylor, Bobova, Zinbarg, Mineka, \& Craske, 2012).

Finally, studies demonstrating PTSD symptom severity to be associated with increased odds of substance use (Vetter, Rossegger, Rossler, Bisson, \& Endrass, 2008) or subsequent changes in SUD symptoms and problems (Langdon et al., 2016; Ouimette, Read, Wade, \& Tirone, 2010) have been used in support of the self-medication hypothesis. For example, Hien and colleagues (2010) investigated temporal relations of PTSD and SUD among women in SUD treatment. Participants were randomly assigned to receive 12 sessions of a trauma-focused or health education intervention, with assessments conducted at pre- and posttreatment, and at 3-, 6, and 12-month follow-up. In addition, substance use and PTSD symptoms were assessed weekly throughout the intervention period. PTSD symptom reductions were associated with subsequent improvements in substance use, but SUD symptom improvement was not associated with subsequent PTSD symptom reduction. Similarly, Ouimette et al. (2010) investigated PTSD and SUD symptoms in a sample of outpatients in SUD treatment. This included assessing symptoms over the previous 26-week period using the Longitudinal Interval Follow-up Evaluation (LIFE; Keller et al., 1987) and the Time Line Follow-Back (Sobell et al., 1980) interviews to document 
weekly changes in PTSD symptoms and daily fluctuations in substance use, respectively. They found a one-unit increase in PTSD symptoms to be associated with a 2.9\%, 7.2\%, and 19\% increase in odds of having alcohol, cocaine, and opioid dependence symptoms the following week, respectively. Change in PTSD status across the 26-week period from remission to full criteria was associated with an $11 \%, 29 \%$, and $94 \%$ relative increase in likelihood of having alcohol, cocaine, and opioid dependence the following week.

Relatedly, PTSD symptoms have been demonstrated to influence subsequent drinking in studies assessing daily PTSD symptoms and alcohol use and associated problems. For instance, Simpson, Stappenbeck, Luterek, Lehavot, and Kaysen (2014) investigated self-medication and mutual maintenance models (i.e., bidirectional relationships between PTSD and alcohol use over time) among a sample of 86 individuals with PTSD and alcohol use disorder using interactive voice response via telephone. PTSD severity was associated with increased same-day alcohol use and alcohol use the following day. Although increased alcohol consumption was associated with same-day PTSD symptoms, alcohol use was not associated with next-day PTSD symptoms as would be indicated by the mutual maintenance or susceptibility models. Gaher and colleagues (2014) also found support for the self-medication model utilizing daily assessments completed by Palm handheld devices. Specifically, 90 veterans completed daily reporting of PTSD symptoms, alcohol consumption, and alcohol problems as prompted at random times throughout the day for an approximately 2-week period. Increased PTSD symptoms in the day were associated with greater alcohol consumption that evening. Greater PTSD symptoms were also associated with subsequent higher alcohol-related problems. Notably, the susceptibility model was not investigated in this study (Gaher et al., 2014). Similar results have been reported among female college drinkers who had experienced a sexual assault as compared to female college 
drinkers with no history of traumatic event exposure (Kaysen et al., 2014). Intrusive and behavioural avoidance symptoms of PTSD (but not arousal or dysphoric symptoms of PTSD) were associated with a higher likelihood of same day alcohol use. Unexpectedly, neither PTSD diagnosis nor trauma exposure moderated the association between PTSD symptoms and alcohol use (Kaysen et al., 2014).

\section{Present Study}

In summary, most individuals experience PTSD-like symptoms subsequent to traumatic exposure that decline over the weeks and months following trauma. However, some individuals continue to experience symptoms and are diagnosed with PTSD. SUD and increased substance use have also been documented as common following trauma exposure. Though less is known about the course of substance use subsequent to trauma, some evidence suggests PTSD may moderate this relationship. With respect to the temporal sequencing of PTSD and substance use, most support has accrued for the self-medication hypothesis, whereby PTSD symptoms precede SUD and increased use of substances, though there is some support for the susceptibility hypothesis.

The above-reviewed research addressing comorbid PTSD/SUD varies in study design, frequently employing cross-sectional and retrospective designs (e.g., Nordløkken et al., 2013; Ouimette et al., 2010), or prospective designs with only two assessment points (Cerdá, Tracy, \& Galea, 2011; McFarlane et al., 2009). There is also variability in relation to the time since traumatic exposure. Study samples are commonly comprised of treatment-seeking individuals with long-standing PTSD (e.g., Back et al., 2006b; Hien et al., 2010), while studies examining PTSD symptoms and substance use more proximal to exposure are typically restricted to individuals hospitalized for traumatic injury (e.g., Zatzick et al., 2002). Moreover, some research 
has not anchored PTSD symptoms to Criterion A traumatic events as required for a diagnosis of PTSD (e.g., Read et al., 2013), therefore limiting generalizability of the results to populations with PTSD.

Additional limitations in this area of research relate to statistical analyses that have been employed to account for associations between PTSD and substance use and SUD over time. Longitudinal studies investigating comorbid PTSD/SUD have typically employed multivariate regression, analysis of variance, hierarchical linear modeling (HLM), or SEM autoregressive cross-lagged designs, all of which answer different questions and have various limitations. For instance, regression and analysis of variance can account for temporal onset when used in prospective longitudinal designs, however these analyses do not accurately account for nested data resulting from repeated-measures designs or growth/decline of a particular variable of interest. Although HLM can answer questions pertaining to growth/decline in a particular variable and appropriately analyze nested data (Raudenbush \& Bryk, 2002), HLM does not allow for the assessment of reciprocal or directional effects between variables over time. Autoregressive cross-lagged designs, on the other hand, can examine reciprocal and directional relationships across time between variables, but are not able to account for differences in variable growth/decline (Ferrer \& McArdle, 2003; King et al., 2006b). Given the trajectory of PTSD symptoms subsequent to trauma is characterized by natural decline in severity, it is imperative that statistical methods account for this natural change over time.

The overall goal of this study was to extend research on the associations between PTSD and SUD by exploring PTSD symptoms and substance use over time among a community sample of individuals exposed to a traumatic event. Using a prospective longitudinal design, this 
sample was recruited within 6 months of their trauma and followed over the course of 1 year. There were three primary aims and associated hypotheses as detailed below.

Aim 1. The first aim of this study was to build on prior research investigating the course of PTSD subsequent to trauma by examining the trajectory of PTSD symptom severity in relation to end-state diagnoses (i.e., PTSD/SUD, PTSD-only, SUD-only, neither disorder). In other words, the aim was to determine if end-state diagnoses moderated the trajectory of PTSD symptoms over time. End-state diagnoses were used rather than diagnoses at initial assessment to (a) replicate past findings documenting PTSD as a disorder of impeded recovery using DSM-5 criteria and (b) investigate the potential role of SUD, which could develop in the months following trauma, as impeding natural recovery from PTSD symptoms.

Hypothesis 1. It was anticipated that there would be an interaction between time since trauma exposure and end-state diagnostic status (PTSD/SUD, PTSD-only, SUD-only, neither disorder), such that PTSD symptoms would evidence the least decline in the PTSD/SUD group, a moderate decline in the PTSD-only group, and the most decline in the SUD-only and neither disorder groups.

Aim 2. The second aim of this study was to explore the trajectories of alcohol and drug use following trauma exposure in relation to end-state diagnoses (i.e., PTSD/SUD, PTSD-only, SUD-only, neither disorder). Despite limited knowledge of the course of these disorders following traumatic exposure, and how the course of substance use might vary based on a diagnosis of PTSD and/or SUD, existing research and theories related to PTSD/SUD following trauma suggests that PTSD is associated with an increase in substance use. Given most prior work has focused on alcohol in absence of drug use, the course of alcohol and drug use were looked at separately in this dissertation. Accordingly, the following hypotheses were put forth: 
Hypothesis $2 a$. It was posited that there would be an interaction between time since trauma exposure and end-state diagnostic status, such that alcohol consumption would increase least among individuals with neither disorder, moderately in the PTSD-only group, and most in the SUD-only and PTSD/SUD groups.

Hypothesis $2 \boldsymbol{b}$. It was posited that there would be an interaction between time since trauma exposure and end-state diagnostic status, such that drug use would increase least among individuals with neither disorder, moderately in the PTSD-only group, and most in the SUD-only and PTSD/SUD groups.

Aim 3. The third aim of this study was to explore the structure of PTSD symptoms and the temporal sequencing of PTSD symptom severity and substance use frequency subsequent to trauma, while accounting for natural change over time.

Hypothesis 3a. It was anticipated that a hierarchical four-indicator measurement model for PTSD consistent with DSM-5 (APA, 2013) PTSD symptom clusters would demonstrate a good fit to the data. Moreover, the hierarchical four-indicator model was expected to demonstrate a better fit to the data as compared to a single-indicator model of PTSD.

Hypothesis 3b. It was anticipated that temporal relationships between PTSD symptom severity and substance use (both alcohol and drug use) would support the self-medication hypothesis. In other words, PTSD symptom severity at one time point (e.g., t1) would predict substance use at the following time point (e.g., t2), while the opposite relationship was not expected (substance use would not predict subsequent PTSD symptom severity).

Hypotheses $3 c$ and $3 d$. When compared against the self-medication model, it was expected that the susceptibility and mutual maintenance models of PTSD symptoms and 
substance use would result in a statistically poorer fit to the data (Hypotheses $3 \mathrm{c}$ and $3 \mathrm{~d}$, respectively).

\section{Method}

\section{Participants}

The present study was derived from a larger longitudinal study investigating risk and resilience factors in the course of PTSD and posttrauma recovery. For the larger study, the majority of individuals were recruited as part of a dyad such that trauma-exposed individuals were asked to invite a significant other (i.e., intimate partner, family member, close friend) to also take part in the study. This significant other had to be aware that the traumatic event occurred but not also exposed to the event. Otherwise eligible individuals who indicated they were unwilling or unable to invite a significant other into the study were permitted to complete the study alone. All trauma-exposed persons, regardless of whether they completed the larger study alone or as part of a dyad, were included in the current study.

The present study included 137 trauma-exposed individuals, $72.26 \%$ of who were women. At the time of their first assessment, participants were on average 37.05 years of age and $62.77 \%$ of individuals were enrolled in an education program or employed. The sample was diverse with respect to ethnicity, and just over half (51.82\%) of participants were single. Excluding PTSD related to the index event associated with study enrollment, $69.34 \%$ of participants endorsed symptoms consistent with any lifetime mental health condition, $66.42 \%$ reported a current mental health condition, and 33.58\% reported a current (past year) alcohol or drug use disorder. Just over one third (37.96\%) of participants were receiving mental health services (e.g., psychotherapy or psychotropic medications). Type of traumatic event associated with enrollment into the study varied, and participants completed their first assessment between 
24 and $260(M=127.14, S D=54.83)$ days following trauma exposure. Additional demographic information for the full sample, as well as separated by participants who completed the study alone or as part of a dyad, is contained in Table 1, while mental health conditions and type of trauma exposure are detailed in Table 2.

\section{Measures}

Clinician-administered and self-report measures relevant to this study are detailed below. These measures were administered at each assessment. All interview measures were administered by trained master's- and doctoral-level clinical psychology students.

Clinician-Administered PTSD Scale. Current PTSD symptom severity and diagnostic status were determined by the Clinician-Administered PTSD Scale (CAPS; Blake et al., 1995). This widely used semistructured interview assesses each of the 17 DSM-IV-TR PTSD symptoms, and additional items were included for use in this study to account for PTSD symptoms as listed in the DSM-5 (i.e., items pertaining to exaggerated and persistent traumarelated negative beliefs, persistent distorted trauma-related blame of self or others, pervasive negative emotional state, and self-destructive or reckless behaviour; APA, 2013). Frequency and intensity are measured for each symptom over the preceding month using a 5-point Likert-scale from 0 (never) to 4 (daily or almost every day) and 0 (none) to 4 (extreme), respectively, with overall PTSD severity computed by summing frequency and intensity scores for all symptoms. A dichotomous PTSD diagnosis was determined based on meeting PTSD symptom cluster criteria (i.e., one intrusion symptom, one avoidance symptom, two negative shifts in cognitions or mood symptoms, and two hyperarousal symptoms), whereby symptoms were considered to be present when they had a frequency rating of at least 1 and an intensity rating of at least 2 (Weathers, Keane, \& Davidson, 2001). The CAPS has demonstrated excellent interrater reliability and 
Table 1

Demographic Information for the Sample at Initial Assessment

\begin{tabular}{|c|c|c|c|}
\hline & $\begin{array}{c}\text { Total Sample }(n=137) \\
n(\%)^{\mathrm{a}}\end{array}$ & $\begin{array}{c}\text { Completed Alone }(n=28) \\
n(\%)^{\mathrm{a}}\end{array}$ & $\begin{array}{c}\text { Completed as Dyad }(n=109) \\
n(\%)^{\mathrm{a}}\end{array}$ \\
\hline Biological Sex (Male) & $38(27.74)$ & $7(25.00)$ & $31(28.44)$ \\
\hline Age $(M[S D])$ & $37.05(14.05)$ & $42.75(14.85)$ & $35.59(13.52)$ \\
\hline \multicolumn{4}{|l|}{ Ethnicity } \\
\hline Black & $19(13.87)$ & $2(7.14)$ & $17(15.60)$ \\
\hline South, South East, East Asian & $23(16.79)$ & $8(28.57)$ & $15(13.76)$ \\
\hline Aboriginal & $6(4.38)$ & $2(7.14)$ & $4(3.67)$ \\
\hline White & $67(48.91)$ & $14(50.00)$ & $53(48.62)$ \\
\hline Mixed or Other Ethnicity & $22(16.06)$ & $2(7.14)$ & $20(18.35)$ \\
\hline \multicolumn{4}{|l|}{ Marital Status } \\
\hline Single & $71(51.82)$ & $10(35.71)$ & $61(55.96)$ \\
\hline Committed Relationship & $21(15.33)$ & $4(14.29)$ & $17(15.60)$ \\
\hline Married & $18(13.14)$ & $5(17.86)$ & $13(11.93)$ \\
\hline
\end{tabular}


Widowed, Separated, Divorced

Employed/ Education

Annual Income

$<\$ 5,000$

$\$ 5,000$ to $\$ 9,999$

$\$ 10,000$ to $\$ 14,999$

$\$ 15,000$ to $\$ 24,999$

$\$ 25,000$ to $\$ 34,999$

$\$ 35,000$ to $\$ 49,999$

$\$ 50,000$ to $\$ 74,999$

$>\$ 75,000$
$25(18.25)$

$86(62.77)$

$29(21.17)$

$15(10.95)$

$19(13.87)$

$23(16.79)$

$15(10.95)$

$18(13.14)$

$8(5.84)$

$7(5.11)$
$9(31.14)$

$16(14.68)$

$68(62.39)$

$8(64.29)$

$5(17.86)$

$24(22.02)$

$12(11.01)$

$12(11.01)$

$18(16.51)$

$12(11.01)$

$16(14.68)$

$7(6.42)$

$6(5.50)$

${ }^{a}$ Data are presented as $n$ s with percentages (\%) unless otherwise indicated.

Note . Employed/education = currently employed or enrolled in an educational program . 
Table 2

Prevalence of DSM-5 Current and Lifetime Mental Health Conditions for the Sample at Initial Assessment and Type of Trauma Exposure Associated with Study Enrolment

$\begin{array}{ccc}\text { Total Sample }(n=137) & \text { Completed Alone }(n=28) & \text { Completed as Dyad }(n=109) \\ n(\%) & n(\%) & n(\%)\end{array}$

\begin{tabular}{|c|c|c|c|}
\hline \multicolumn{4}{|l|}{ Current Mental Health Condition } \\
\hline PTSD (based on CAPS) & $73(53.28)$ & $17(60.71)$ & $56(51.38)$ \\
\hline Alcohol Use Disorder & $33(24.09)$ & $7(25.00)$ & $26(23.85)$ \\
\hline Drug Use Disorder & $27(19.71)$ & $4(14.29)$ & $23(21.10)$ \\
\hline PTSD (related to another event) & $20(14.60)$ & $4(14.29)$ & $16(14.68)$ \\
\hline Mood Disorder & $60(43.80)$ & $16(57.14)$ & $44(40.37)$ \\
\hline Anxiety Disorder & $35(25.55)$ & $9(32.14)$ & $26(23.85)$ \\
\hline Psychotic Disorder & $2(1.46)$ & $0(0.00)$ & $2(1.83)$ \\
\hline Eating Disorder & $6(4.38)$ & $1(3.57)$ & $5(4.59)$ \\
\hline \multicolumn{4}{|l|}{ Lifetime Mental Health Condition } \\
\hline PTSD (related to another event) & $26(18.98)$ & $6(21.43)$ & $20(18.35)$ \\
\hline
\end{tabular}




$\begin{array}{lccc}\text { Mood Disorder } & 79(57.66) & 21(75.00) & 58(53.21) \\ \text { Anxiety Disorder } & 39(28.47) & 9(32.14) & 30(27.52) \\ \text { Psychotic Disorder } & 3(2.19) & 0(0.00) & 3(2.75) \\ \text { Type of Traumatic Event } & & & 24(22.02) \\ \text { Sexual Assault } & 32(23.36) & 8(28.57) & 27(24.77) \\ \text { Physical Assault } & 34(24.82) & 7(25.00) & 34(31.19) \\ \text { Accident } & 39(28.47) & 5(17.86) & 10(9.17) \\ \text { Sudden Illness or Death } & 14(10.22) & 4(14.29) & 4(3.67) \\ \text { Robbery or Home Invasion } & 7(5.11) & 3(10.71) & 10(9.17) \\ \text { Other Trauma } & 11(8.03) & 1(3.57) & \end{array}$

Note. $\mathrm{PTSD}=$ posttraumatic stress disorder; CAPS $=$ Clinician Administered PTSD Scale. 
internal consistency across a range of settings and populations. In addition, test-retest reliability has been reported in the high range and strong convergent validity with other measures of PTSD has been noted (Blake et al., 1995; Foa \& Tolin, 2000; Weathers et al., 2001; Weathers, Ruscio, \& Keane, 1999).

For this study, the CAPS was specifically anchored to the traumatic event experienced within the 6 months prior to study enrollment. In addition, each CAPS administration included an assessment of the event to ensure it met criteria for a Criterion A trauma. Internal consistency for the CAPS in this study ranged from good to excellent across all assessment points for total severity ( $\alpha \mathrm{s}=.95$ to .96$)$, intrusion symptoms ( $\alpha \mathrm{s}=.85$ to .92$)$, avoidance $(\alpha \mathrm{s}=.80$ to .90$)$, negative shifts in cognitions and mood $(\alpha \mathrm{s}=.89$ to .92$)$, and hyperarousal symptoms $(\alpha \mathrm{s}=.86$ to .89). To assess interrater reliability of the clinician assessors, a random sample of 34 CAPS administrations ( $7.31 \%$ of CAPS administrations completed at the time this dissertation was conducted) were evaluated by an independent clinical psychologist who listened to the audiorecorded assessments. The kappa coefficient pertaining to DSM-5 PTSD diagnostic status was very good $(\kappa=.90)$ between the clinician assessors and the independent assessment monitor. Furthermore, intraclass correlation coefficients (ICC) between the clinician assessors and the independent assessment monitor were excellent for total severity ( $\mathrm{ICC}=.99)$, as well as reexperiencing $(\mathrm{ICC}=.99)$, avoidance $(\mathrm{ICC}=.98)$, negative shifts in cognitions and $\operatorname{mood}(\mathrm{ICC}=$ $.99)$, and hyperarousal $(\mathrm{ICC}=.99)$ cluster scores. ICC was calculated using a 2-way mixed with absolute agreement approach.

Mini International Neuropsychiatric Interview. The Mini International Neuropsychiatric Interview (MINI; Lecrubier et al., 1997) was used to determine past and current Axis I psychiatric disorders other than PTSD, as well as PTSD related to other prior or 
intervening traumatic events than that assessed with the CAPS. PTSD diagnostic status pertaining to the traumatic event associated with study enrollment was determined based on the CAPS. Items were added to supplement the MINI for this study to assess DSM-5 (APA, 2013) disorder criteria. For SUDs, this included addition of the cravings criterion and assessing for marijuana-related withdrawal. The MINI has demonstrated high concordance with the Structured Clinical Interview for DSM-IV Disorders (e.g., Jones et al., 2005).

Frequency of Drug and Alcohol Use. Substance use was measured using a series of drug- and alcohol-related self-report questions about use within the past month. Two items pertained to alcohol use. The first asked "how often during the last month did you usually have any kind of beverage containing alcohol, whether it was wine, beer, whiskey, or any other drink?", which was on a 7-point Likert-scale (i.e., never, about once a month, several times a month, 1-2 days a week, 3-4 days a week, 5-6 days a week, everyday). The second item asked participants to indicate "on those days you drank beer, wine, or hard liquor during the last month, how many drinks did you usually have per day?" These alcohol use questions correspond with alcohol frequency and quantity items used in prior research (e.g., Cahalan, Cissin, \& Crossley, 1969; Richman, Shannon, Rospenda, Flaherty, \& Fendrich, 2009); however, no psychometric data have been published on these items. For this study, frequency of alcohol consumption in the past month was determined by multiplying the number of drinks consumed on drinking days by the number of drinking days in the past month. To achieve this, the Likert-scale item was modified to reflect the estimated number of past month drinking days (i.e., never $=0$, about once a month $=1$, several times a month $=3,1-2$ days a week $=6,3-4$ days a week $=14,5-6$ days $a$ week $=22$, everyday $=30$. 
With respect to drug use, frequency of use for a range of drugs was assessed on a 7-point scale (never, about once a month, several times per month, 1-2 times per month, 3-4 times per month, 5-6 times per month, everyday). These items correspond to the standard drug classes, with specific items pertaining to use of cannabis; hallucinogens; phencyclidine; inhalants; opioids; sedatives, hypnotics, and anxiolytics; stimulants; and cocaine. Each question inquiring about frequency of drug use was followed by two additional items related to prescription use, in terms of whether the drug was prescribed, and whether the drug was taken in greater amounts than prescribed. For the purposes of this study, total frequency of drug use was determined by (a) modifying Likert-scale items to reflect estimated days using each type of drug (i.e., never $=0$, about once a month $=1$, several times a month $=3,1-2$ days a week $=6,3-4$ days a week $=14$, $5-6$ days a week $=22$, everyday $=30$ ), (b) recoding items where participants indicated that they used a particular drug as prescribed to 0 , and (c) summing each item pertaining to drug use to create a total frequency score. Thus, drug use in this dissertation included both illicit drug use (e.g., heroin) and abuse of prescription medications (e.g., morphine).

Mental Health Utilization Questionnaire. Utilization of psychosocial and psychopharmacological treatments was assessed with the Mental Health Utilization (MHU) measure. This clinician-administered interview asks about the type of mental health treatment and frequency of use, as well as information pertaining to the mental health provider and specific aspects of the service in terms of the methods of care employed. Though no psychometric data

for this measure are available, variations of this measure have been used in research pertaining to PTSD (e.g., Schnurr et al., 2007). For this study, the MHU was used to describe the sample.

\section{Procedure}

Participants were recruited by way of online advertisements, flyers posted throughout the 
community, newspaper advertisements, and referrals from partnering hospitals and mental health service providers in the Greater Toronto Area (GTA). Individuals who contacted study personnel were informed of the details of participation and screened via telephone for preliminary inclusion criteria. To be eligible, participants had to (a) be between the ages of 18 and 75 years, (b) give consent to have their assessments audio-recorded, (c) be fluent in spoken and written English, and (d) have experienced a DSM-5 (APA, 2013) PTSD Criterion A traumatic event within the past 6 months. In an effort to increase external validity, there were no additional inclusion/exclusion criteria.

In the larger study, potential participants were asked to invite a close other (intimate partner, family member, or close friend) to complete the study with them, and they were further informed that this was a requirement for study inclusion. Individuals who indicated that they (a) did not have a close other to participate or (b) were unwilling to include a close other in the study were given 48 hours to identify a close other with whom to complete the study. After this 48 hour period, potential participants were recontacted by study staff and invited to participate in the study alone if they remained unable or unwilling to enroll with a close other. The larger study aimed specifically to investigate relationships after trauma exposure between dyads; thus, individuals were only given the option to participate alone once they refused to participate at second contact due to lack of close other to invite. Since the current study included only traumaexposed individuals, regardless of whether they completed the study alone or with a close other, the remainder of the procedure is specific to the trauma-exposed participants.

Those who screened positive for the inclusion criteria, then signed the informed consent form and were scheduled to complete their first assessment. Participants were assessed approximately every 4 months for a total of four assessments over the course of a year. Each 
assessment included a clinical interview comprised of the CAPS, MINI, and MHU, as well as a self-report component that included the frequency of drug and alcohol use questionnaire and demographic information. Individuals first completed the interview portion of the assessment, followed by the self-report questionnaire package that they were required to complete within one week of the interview. Limits of confidentiality were reviewed with participants prior to each assessment interview. Participant flow throughout the course of the study is depicted in Figure 1.

Participants were given the option of completing the assessments in-person at Ryerson University or remotely by completing the interview over the telephone and the self-report questionnaires online using the survey administration software Qualtrics (Qualtrics, 2005). Compensation was provided to participants for completing the assessments, with a graduated remuneration schedule employed. Specifically, participants were paid $\$ 60$ for completing the first assessment, $\$ 70$ for the second, $\$ 80$ for the third, and $\$ 90$ for completing the fourth assessment. Approval for this protocol was obtained from the Research Ethics Board at Ryerson University.

\section{Data Analytic Strategy}

Descriptive statistics and bivariate analyses were first conducted to assess simple relationships between all variables of interest. To address study hypotheses, two types of analyses were performed: Growth curve modeling (GCM) using the HLM7 software package (Raudenbush, Bryk, \& Congdon, 2011) and bivariate latent difference score (BLDS) structural equation modeling (SEM) using the Mplus software package (Version 7.11; Muthén \& Muthén, 1998-2013). Prior to conducting these models, univariate normality was assessed. If skew or kurtosis were not within acceptable ranges [i.e., skew above $|3|$ and kurtosis above $|7|$ (Kline, 2011) or absolute $z$ values for skew and kurtosis above 3.29 (Kim, 2012; Tabachnick \& Fidell, 


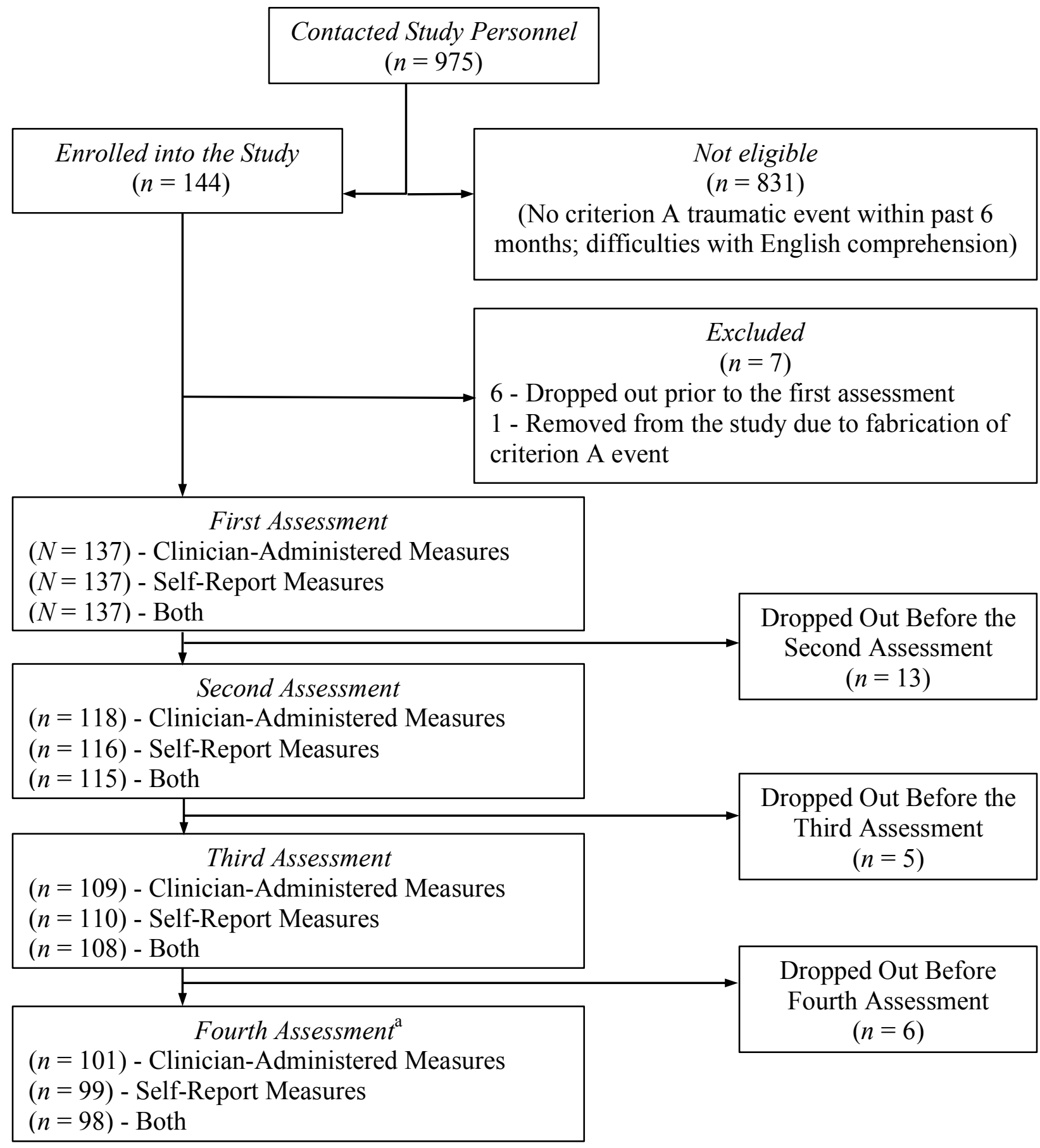

Figure 1. Consort diagram depicting participant flow through the study.

${ }^{\mathrm{a}}$ The study concluded prior to nine participants having the opportunity to complete their fourth assessment. 
2007)], variables were transformed following the recommendations of Tabachnick and Fidell (2007). Normality and transformations for GCM and BLDS analyses are detailed below.

Growth Curve Modeling: Aims 1 and 2. Study hypotheses involving change over time in PTSD symptoms and substance use by diagnostic status were investigated using a series of growth curve models. Growth curve modeling, an application of multilevel modeling, is a useful technique for panel data because it provides information regarding trajectory of change (growth or decline) for a particular variable of interest by estimating an underlying trajectory for each individual within the sample. For this analysis, change is a function of the variable intercept (average initial starting value), variable slope (average individual rate of change over time), and random error (Raudenbush \& Bryk, 2002). Growth curve modeling takes into account systematic shared variance between nested data, thereby compensating for violations of independence of observations (Raudenbush \& Bryk, 2002). This approach also permits estimation of missing data at Level-1 and has the capacity to handle unbalanced designs, including variation among assessment times between subjects (Tabachnick \& Fidell, 2007).

For the growth curve models in this study, a 2-level structure was used with assessment time points (Level-1) nested within individuals (Level-2). Random intercepts and slopes were specified to account for individual differences in the predictors (PTSD symptom severity, alcohol use, drug use), and time was treated as continuous. Specifically, a time variable was calculated based on the number of days elapsed since trauma exposure at which each assessment occurred, and this time variable was centered on the date of traumatic event exposure. Analyses were performed using full maximum likelihood estimation.

To determine the effects of end-state diagnostic status (i.e., PTSD diagnosis and/or SUD diagnosis at final assessment point) on the course of PTSD symptom severity, as per Aim 1, an 
initial growth curve model was evaluated with time at Level-1 predicting PTSD total symptom severity. Subsequently, two dummy variables reflecting PTSD and SUD diagnostic status at the final assessment were entered into the model to investigate differences in the trajectory of PTSD symptom severity posttrauma, and initial PTSD severity, based on the presence or absence of PTSD or SUD. Dummy variables were entered at Level-2 for both the intercept and slope. Similar models were run for alcohol and drug use, in accordance with Aim 2, to determine the effects of end-state diagnostic status on the trajectory of substance use following trauma.

Since growth curve modeling assumes variables are normally distributed, univariate normality was assessed prior to performing analyses. As detailed in Table 3, PTSD total scores were within recommended ranges for absolute values of skew and kurtosis; however, several of the $z$ scores were above 3.29. Thus, a square root transformation $(\sqrt{ } \mathrm{x})$ was performed on the PTSD total severity variables, which improved the distributions. Most of the substance use variables had skew above $|3|$ and kurtosis above $|7|$, as well as absolute $z$ values above 3.29 (Table 3). Conducting inverse transformations $(1 /(1+x))$ improved the distributions for alcohol and drug use variables for all assessment points based on the recommended absolute values of skew and kurtosis (Kline, 2011). Although, based on $z$ scores, several of the alcohol use variables still exhibited kurtosis (zs ranging from $|2.79|$ to $|3.78|$ ) and the drug use variables still exhibited skew (zs ranging from $|5.33|$ to $|5.79|$ ) subsequent to inverse transformation, growth curved models were conducted with these variables as full maximum likelihood estimation with robust standard errors is considered robust against model misspecification and non-normality (Garson, 2013). Given the significance levels of all models with nontransformed variables were not markedly different from their corresponding model with transformed variables, data from the nontransformed models are reported below to aid interpretability of the findings. 
Table 3

Skew and Kurtosis for Original and Transformed Variables Used in Growth Curve Models Related to Aims 1 and 2

\begin{tabular}{|c|c|c|c|c|c|c|c|c|c|c|c|c|}
\hline \multirow{3}{*}{ Variables } & \multicolumn{6}{|c|}{ Original Variables } & \multicolumn{6}{|c|}{ Transformed Variables } \\
\hline & \multicolumn{3}{|c|}{ Skew } & \multicolumn{3}{|c|}{ Kurtosis } & \multicolumn{3}{|c|}{ Skew } & \multicolumn{3}{|c|}{ Kurtosis } \\
\hline & Est. & $S E$ & $z$ & Est. & $S E$ & $z$ & Est. & $S E$ & $z$ & Est. & $S E$ & $z$ \\
\hline t1 PTSD Total & 0.20 & 0.24 & 0.85 & -0.42 & 0.48 & -0.89 & -0.77 & 0.24 & -3.20 & 0.11 & 0.48 & 0.22 \\
\hline t2 PTSD Total & 0.91 & 0.25 & 3.70 & 0.32 & 0.49 & 0.66 & -0.16 & 0.25 & -0.64 & -0.61 & 0.49 & -1.27 \\
\hline t3 PTSD Total & 1.35 & 0.25 & 5.36 & 1.39 & 0.50 & 2.79 & 0.27 & 0.25 & 1.06 & -0.80 & 0.50 & -1.60 \\
\hline t4 PTSD Total & 1.79 & 0.24 & 7.47 & 2.94 & 0.48 & 6.17 & 0.57 & 0.24 & 2.39 & -0.48 & 0.48 & -1.01 \\
\hline t1 Alcohol Use & 4.74 & 0.25 & 18.87 & 28.00 & 0.50 & 56.22 & 0.17 & 0.25 & 0.69 & -1.77 & 0.50 & -3.56 \\
\hline t2 Alcohol Use & 3.04 & 0.25 & 12.00 & 10.97 & 0.50 & 21.94 & 0.25 & 0.25 & 1.00 & -1.73 & 0.50 & -3.46 \\
\hline t3 Alcohol Use & 7.33 & 0.26 & 28.42 & 59.79 & 0.51 & 117.00 & 0.45 & 0.26 & 1.72 & -1.42 & 0.51 & -2.79 \\
\hline t4 Alcohol Use & 5.57 & 0.25 & 22.38 & 35.79 & 0.49 & 72.60 & -0.05 & 0.25 & -0.22 & -1.87 & 0.49 & -3.78 \\
\hline t1 Drug Use & 3.18 & 0.25 & 12.93 & 10.45 & 0.49 & 21.41 & -1.31 & 0.25 & -5.33 & -0.18 & 0.49 & -0.37 \\
\hline t2 Drug Use & 2.93 & 0.25 & 11.90 & 10.43 & 0.49 & 21.38 & -1.42 & 0.25 & -5.79 & -0.09 & 0.49 & -0.19 \\
\hline t3 Drug Use & 3.41 & 0.25 & 13.43 & 13.08 & 0.50 & 26.01 & -1.36 & 0.25 & -5.37 & -0.02 & 0.50 & -0.04 \\
\hline
\end{tabular}


Note. Sample used for these analyses included only those participants who had completed their time 4 assessment $(n=101)$ as this was needed to determine diagnostic status at the last assessment occasion. PTSD variables were modified by conducting square root transformations $(\sqrt{ } \mathrm{x})$ and substance use variables were modified by conducting inverse transformations $(1 /[1+\mathrm{x}])$. PTSD $=$ posttraumatic stress disorder. 


\section{Bivariate Latent Difference Score Structural Equation Modeling: Aim 3. Temporal}

sequencing between PTSD symptom severity and substance use was investigated with bivariate latent difference score SEM. BLDS modeling offers several benefits over data analytic strategies used to date with longitudinal data, including improved accuracy of estimates due to the use of latent variables and the incorporation of dynamic change related to both natural growth/decline (i.e., "nonstationarity" or "constant change") and autoregressive effects (i.e., "proportional change"). Constant change refers to mean level change in a given variable as a function of time, while proportional change represents the effect of prior status of a given variable on subsequent scores for the same variable (Ferrer \& McArdle, 2003; McArdle, 2001). In BLDS models, latent change is conceptualized as systematic growth or decline over time that may be either accelerated or decelerated based on the previous state of both model constructs (Little, 2013). For instance, change in PTSD symptom severity at Time 2 in the present study is predicted from natural decline in PTSD symptom severity subsequent to traumatic exposure in addition to the influence of PTSD symptom severity at Time 1 and substance use at Time 1.

One limitation of BLDS modeling, and SEM more generally, is the dependence on timestructured data (Willet \& Sayer, 1994). Specifically, SEM requires constraints across participants' assessment schedules such that all participants have a common time for each measurement occasion. However, in the current study participants differed with respect to timing of the first assessment in relation to the occurrence of traumatic exposure, as well as dispersion of follow-up assessment timing relative to targeted assessment times. Thus, the data were first restructured according to the procedures outlined by King et al. (2006a). Implementing this procedure results in the redistribution of data into time segments or time bins that can be imposed on the data and compared to the standard occasions of measurement (e.g., Time 1, Time 2, etc.). 
The purpose of restructuring the data in this way is to minimize differences between individual participants' times of assessments and those assessment times assigned to the entire sample, while maximizing the number of covariances calculated between variables by time segments; thus leading to increased accuracy of parameter estimates and enhanced model fit (King et al., 2006a).

Using the methods detailed in King et al. (2006a), three time structures based on the number of days since trauma exposure were developed and tested. The first was an occasions of measurement time structure that used the mean time since trauma for the four assessment occasions. For the second and third time structure, the data were redistributed into time segments that were chronologically tied to the date of traumatic exposure, yielding a five segment time structure of approximately 4-month intervals (i.e., Time $1 \leq 120$ days; Time $2=121-240$ days; Time $3=241-360$ days; Time $4=361-480$ days; Time $5 \geq 481$ ) and a six segment time structure of approximately 3 -month intervals (i.e., Time $1 \leq 90$ days; Time $2=91-180$ days; Time $3=$ 181-270 days; Time $4=271-360$ days; Time $5=361-450$ days; Time $6 \geq 451$ ). Total PTSD symptom severity as measured by the CAPS was used as the outcome variable to evaluate the time structures. As such, each participant's scores for PTSD severity were systematically placed within one, two, three, or four of the time segments as based on the number of assessments completed by the individual. Restructuring the data in this way resulted in instances in which participants' scores for two assessments fell within the same time segment. To maximize the amount of data retained when two assessments fell within the same time segment, data from the assessment that was closest in number of days to an adjacent empty time segment were shifted to that empty time segment. If there were no empty adjacent time segments, or if both assessment points were more than half the distance from adjacent empty time segments (i.e., $61+$ days for 
the 5 time segment structure or $46+$ days for the 6 time segment structure), data from the two assessments falling within the same time segment were averaged.

Growth curve models were run for each of the time structures to assess the deviance statistic and determine the time structure that best fit the data. For each model, time was centered on the date of traumatic event exposure, with random intercepts and slopes specified (i.e., the intercept and slope of predictors were permitted to vary across participants). The deviance statistic is a goodness-of-fit indicator that is a function of the log-likelihood of the data for the model compared to a saturated model, with larger deviance indicative of poorer model-data fit. The deviance permits a good comparison between models given the same data were used across each of the alternative time structures with models only differing based on the representation of time (King et al., 2006a).

Using the time structure that best fit the data, BLDS analyses were conducted using a two-step modeling approach. First, a series of univariate latent difference score (LDS) models were constructed and compared for each outcome variable (i.e., PTSD total severity, PTSD intrusion, PTSD hyperarousal, PTSD avoidance, PTSD negative shifts in cognitions and mood, alcohol use, and drug use) to determine the model that best fit the data for each outcome variable with respect to representation of change over time. All outcome variables were represented by single-indicator latent variables within the models, which is common in BLDS modeling (Hawley, Ho, Zuroff, \& Blatt, 2006; King et al., 2006a; Nickerson et al., 2014). Second, BLDS models were constructed using the best fitting univariate LDS models to examine temporal relations between PTSD symptom severity and substance use. The full information maximum likelihood (FIML) method of estimation was used for incomplete data under the assumption that data were missing at random for all univariate LDS and BLDS models. Further, residual 
measurement error variances were constrained to be equal within constructs and any nonstationarity effects were fixed to 1 .

For PTSD total severity, an additional step was carried out prior to running the BLDS models to establish whether the latent construct of PTSD symptom severity was better captured by its symptoms clusters (i.e., four-indicator latent factor comprised of CAPS PTSD cluster total scores) or a single indicator latent factor (i.e., single-indicator CAPS total severity score). Figure 2 contains a visual depiction of the hierarchical four-indicator PTSD model. The symbols used in this figure are standard for SEM, whereby latent (unmeasured or unobserved) factors are represented by circles or ellipses, indicator (measured or observed) variables are represented by squares or rectangles, direct (causal) relationships are depicted by single headed arrows (i.e., regression coefficients), and covariances are depicted by double headed arrows. Measurement error factors are denoted by " $e$ " within the factor label.

Univariate LDS models for outcome variables. For each outcome variable (i.e., PTSD total severity, PTSD intrusion, PTSD hyperarousal, PTSD avoidance, PTSD negative shifts in cognitions and mood, alcohol use, and drug use), six univariate LDS models that varied based on the representation of change over time were constructed and compared. Specifically, these models differed on whether they incorporated constant (nonstationarity) change and/or proportional (autoregressive) change, as well as whether proportional change was constrained to equality or permitted to vary across time. Comparative model-to-data fit of the univariate LDS models for each of the outcome variables was determine using the model fit indices detailed below.

As depicted in Figure 3, the dual variable change model incorporates both constant change and proportional change that is free to vary (Panel A). Both constant and proportional 


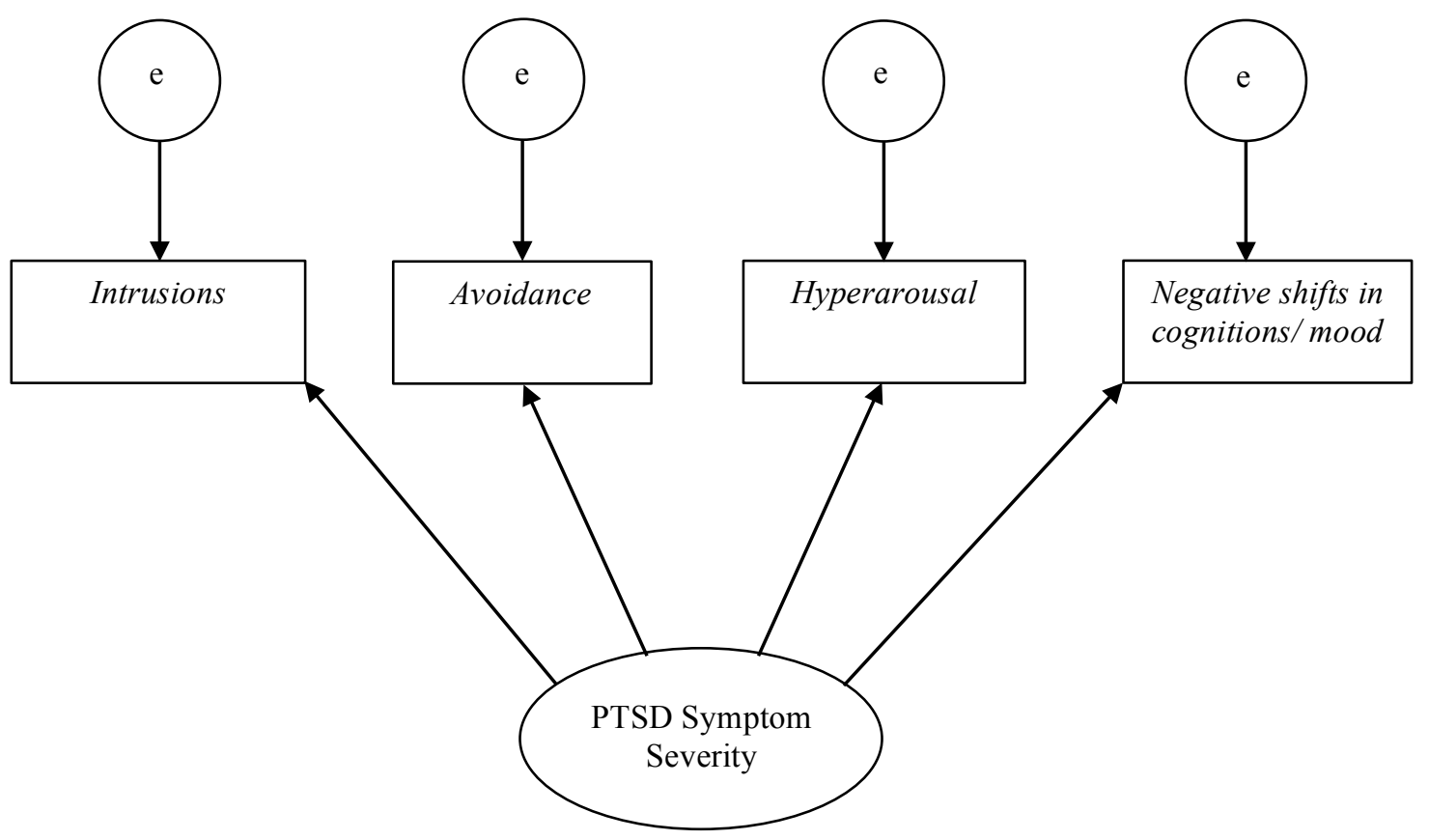

Figure 2. Hierarchical four-indicator measurement model of PTSD symptom severity, with indicators based on DSM-5 PTSD symptom clusters. Latent factors labeled with es represent measurement errors associated with each of the PTSD symptom cluster indicators. 
changes are also incorporated into the dual fixed change model, though proportional change is constrained to equality (Panel B). Constraining proportional change to be equal across time fixes the effects of prior status of a variable on the subsequent score for that variable to be consistent across all assessment occasions, rather than allowing the effect of prior status on the subsequent score to vary across assessment occasions. A proportional change model results when constant change is not a necessary feature for good model fit and constant change is dropped from the model. The variable proportional change (Panel C) and fixed proportional change (Panel D) models suggest that change in the variable across time is the sole function of prior status, where the influence of prior status is permitted to vary across time in the variable proportional change model and is constrained to be equal across time in the fixed proportional change model. In a similar manner, when proportional change is not a relevant feature for good model fit, only constant change is retained in the model resulting in a constant change model (Panel E). The constant change model suggests that only natural growth/decline contributes to change in a variable across time. In the no change model, neither component of change is a necessary feature for good model fit and, thus, any change in the variable across time is attributed to random error (Panel F).

While full maximum likelihood estimation is robust to nonnormality, there were marked deviations from the normal distribution for both alcohol (skew ranging from 2.84 to 8.03 , kurtosis ranging from 8.80 to 69.15 ) and drug use frequency items (skew ranging from 3.36 to 5.15, kurtosis ranging from 11.24 to 30.04 ). Thus, drug and alcohol frequency items were transformed for latent difference score analyses using inverse transformations $(1 /[1+\mathrm{x}])$ that improved the distributions. It was not necessary to transform the PTSD variables. 
A) Dual Variable Change Model

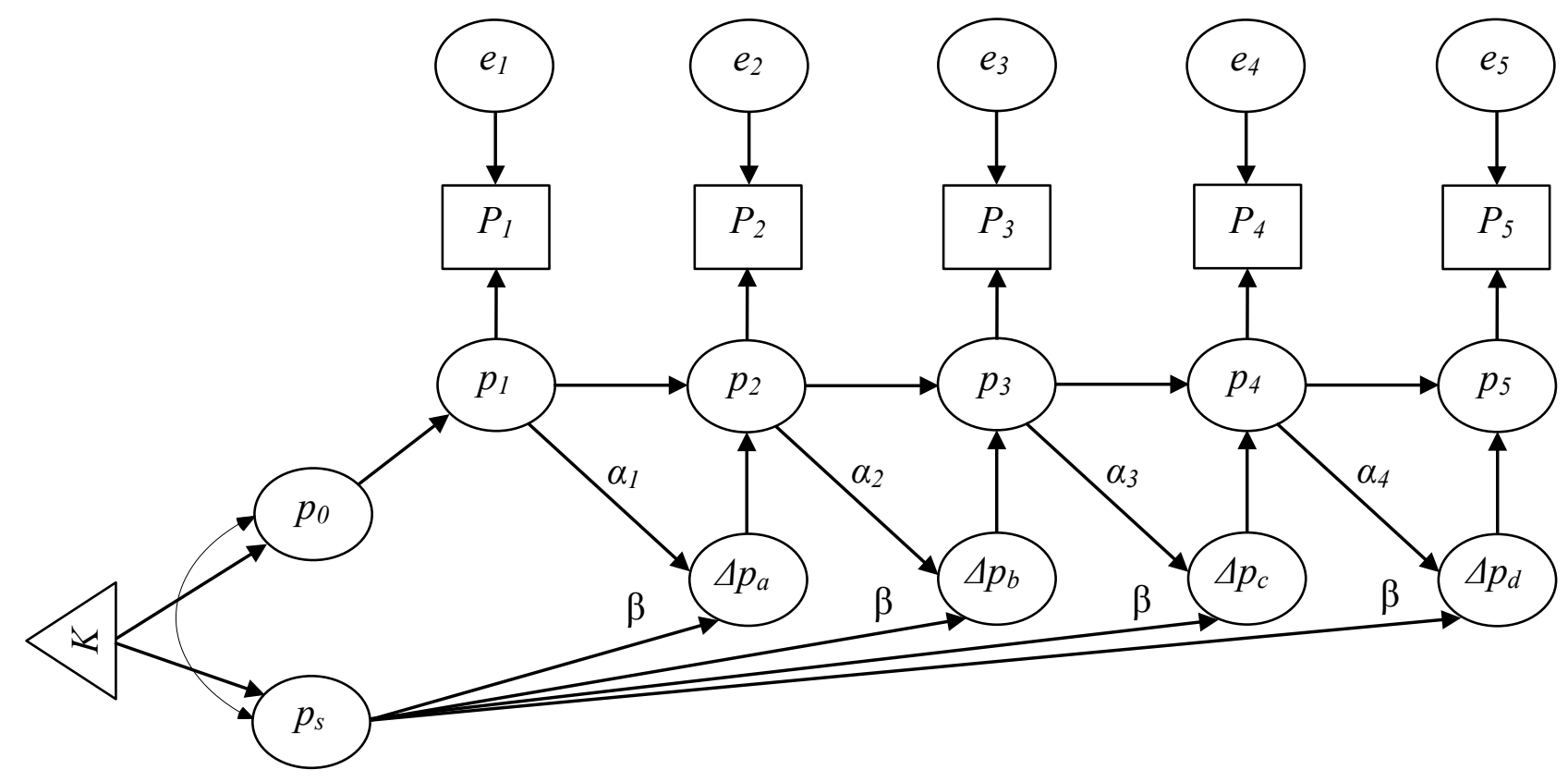

B) Dual Fixed Change Model

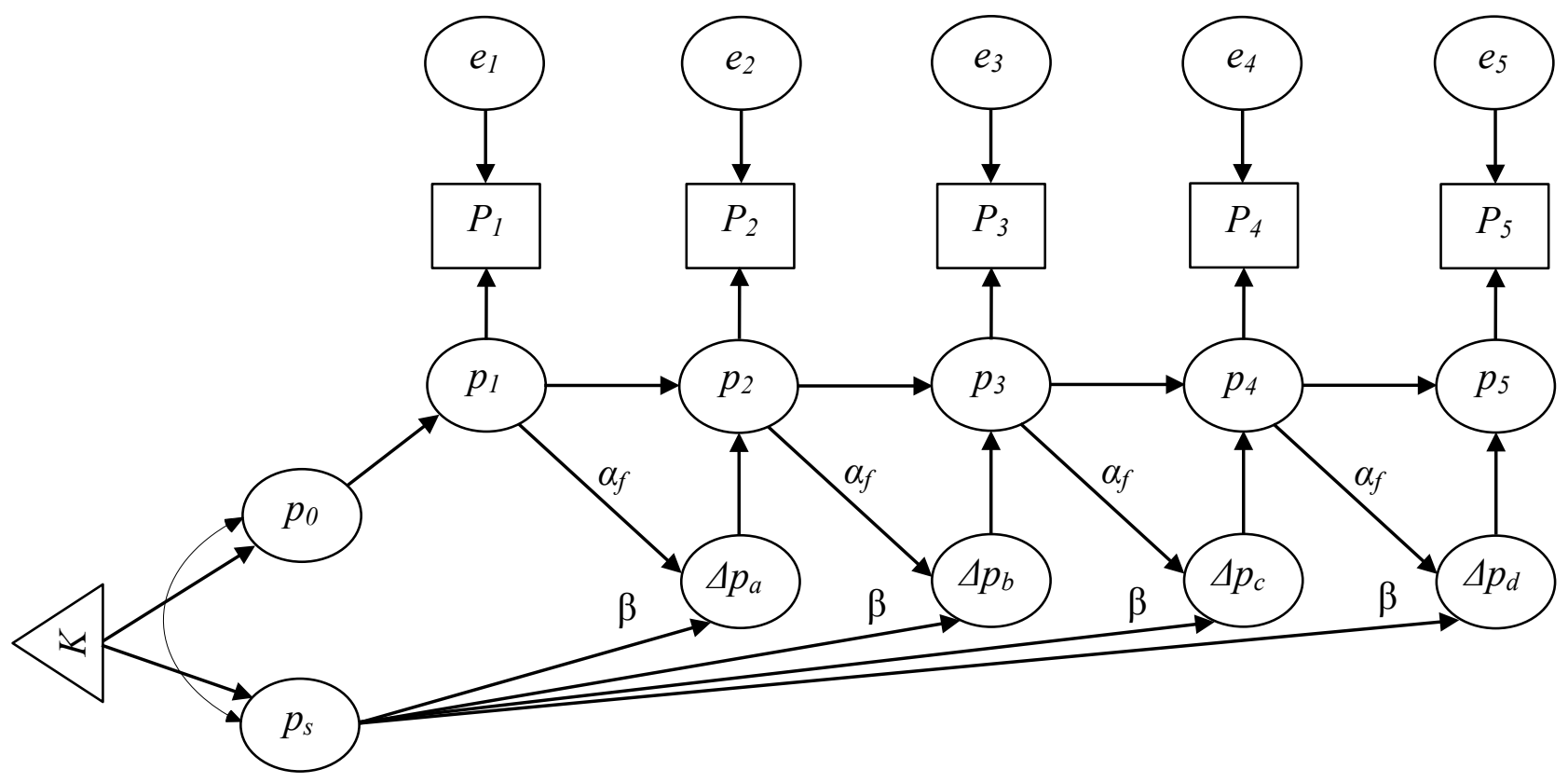


C) Variable Proportional Change Model

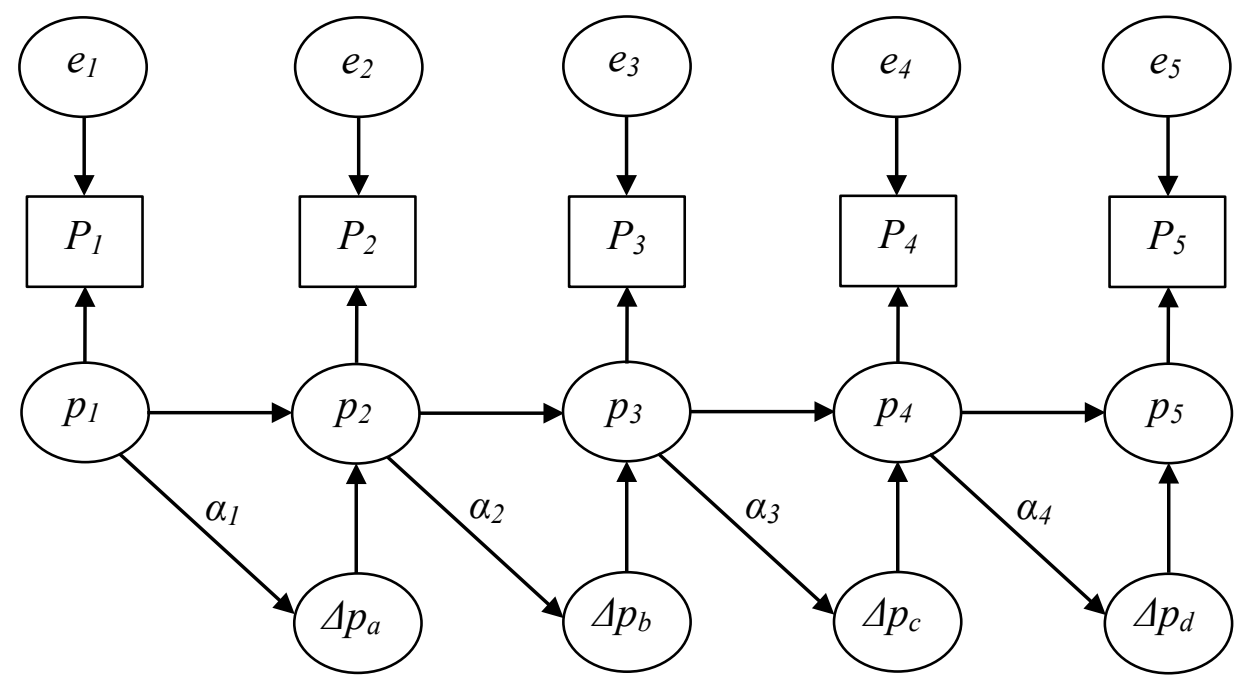

D) Fixed Proportional Change Model

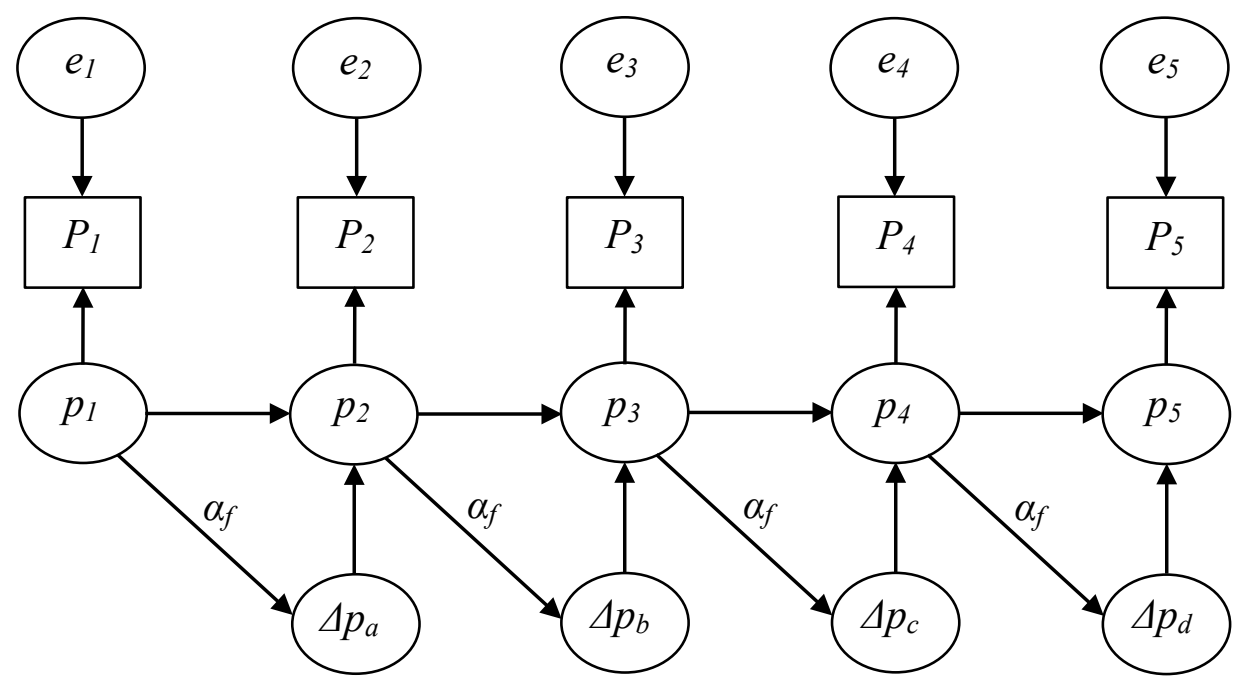


E) Constant Change Model

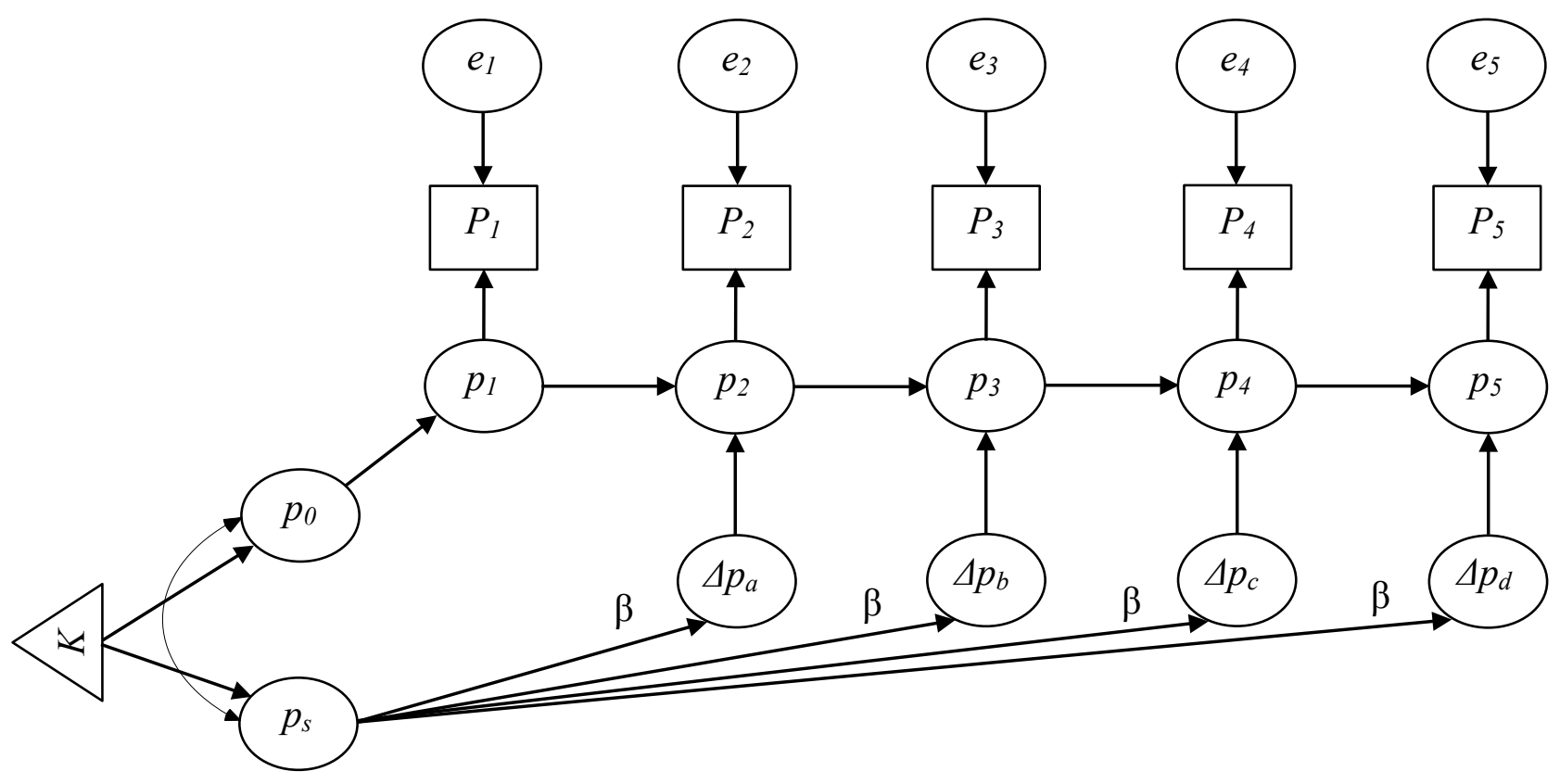

F) No Change Model

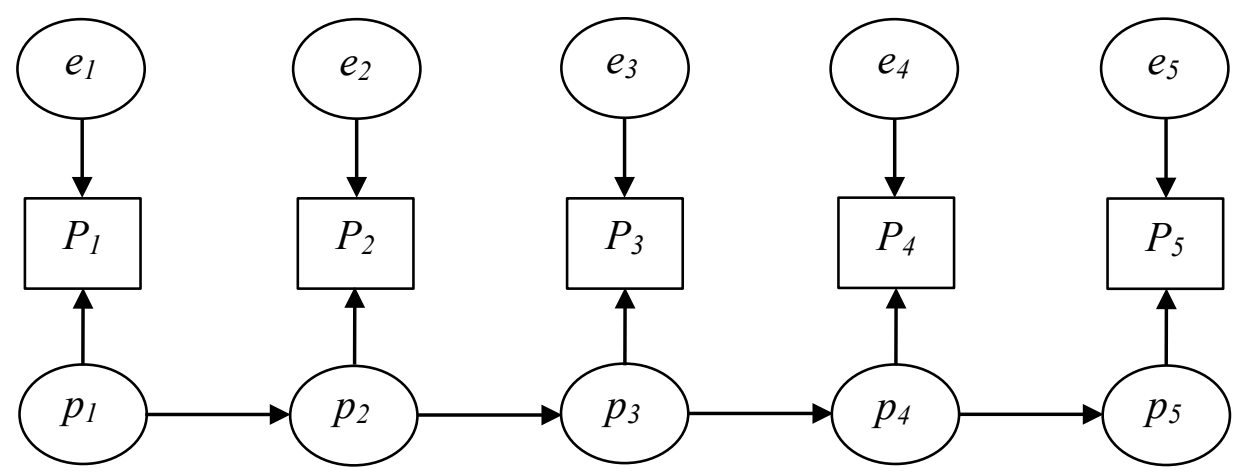

Figure 3. Path diagrams for the six univariate latent difference score models based on the 120day time structure using PTSD as the construct of interest. $P_{[1-4]}$ denotes the observed PTSD scores at each timepoint, $p_{[t]}$ represents the true score latent variables for each timepoint, $\Delta p_{[a-d]}$ represents the latent difference scores between corresponding time points, $p_{0}$ denotes the 
estimated initial status mean for PTSD, $p_{\mathrm{s}} \mathrm{x} \beta \mathrm{s}$ represents the effect of PTSD nonstationarity (i.e., constant change) on PTSD latent difference scores, $\alpha_{[1-4]}$ represents the proportional change as a functioning of the prior true score latent variable PTSD status on the subsequent latent difference score, and $K$ represents a constant used to estimate means and intercepts. $e_{\mathrm{p}}$ represents the error terms for PTSD, which were constrained to be equal across timepoints. 


\section{Measurement model and univariate LDS models for PTSD total symptom severity:}

Testing Hypothesis 3a. Consistent with Hypothesis 3a, a measurement model was specified with confirmatory factor analysis (CFA) to determine the degree to which the latent construct of PTSD total symptom severity was captured by its corresponding symptom clusters. The measurement model consisted of a four-indicator latent factor reflecting the DSM-5 symptom clusters, with indicators comprised of the CAPS intrusions, CAPS hyperarousal, CAPS avoidance, and CAPS negative shifts in cognitions and mood scores (see Figure 2). Error variances for each indicator were constrained to be equal across assessment occasions as is suggested for longitudinal analyses incorporating a panel design (McArdle, 2007). Model fit of the four-indicator measurement model, was determined based on multiple goodness-of-fit indices (detailed below). This four-indicator measurement model was then combined with each of the six univariate LDS structural models and compared to the corresponding univariate LDS singleindicator model for PTSD total score. For instance, the four-indicator no change model for PTSD total score and the single-indicator no change model for PTSD total score were compared, as were the single- and four-indicator constant change models for PTSD total score, and so forth. Determination of whether the BLDS models incorporating PTSD total symptom severity would include the single- or 4-indicator measurement model was based on selection of the univariate LDS model demonstrating the best model fit.

While single-indicator measurement models can be specified with CFA, this approach was not taken in the current study to compare against the four-indicator measurement model for PTSD total symptom severity. Notably, in order to identify a single-indicator construct it is necessary to place a further restriction on the model in addition to fixing the loading of the indicator to be 1, which could be done by either fixing the residual error to be 0 (i.e., presuming 
the indicator is measured without error) or making an informed guess about the amount of measurement error expected based on prior studies and applying the Spearman-Brown prophecy formula (Little, 2013). However, conventional LDS modeling with single-indicator constructs do not place any restrictions on the measurement component of the model beyond fixing the loading of the indicator to be 1 (e.g., King et al., 2006a; Nickerson et al., 2014). Additionally, placing a further restriction on the model would result in estimation that is limited based on the assumption selected (Little, 2013). As such, rather than comparing their measurement models, the fourindicator latent variable model for PTSD total symptom severity was compared to the singleindicator latent variable model for PTSD total symptom severity by directly evaluating their model fits for each of the abovementioned 6 univariate LDS models.

\section{Constructing and testing BLDS models using best-fit univariate LDS models for}

each outcome variable: Testing hypotheses $3 \mathbf{b}$ through 3d. Separate BLDS models were fit to the data using drug use frequency and alcohol consumption that were each combined with PTSD total severity, PTSD intrusion, PTSD hyperarousal, PTSD avoidance, PTSD negative shifts in cognitions and mood, thus resulting in 10 BLDS models. Figure 4 provides a visual depiction of a BLDS model incorporating dual variable change for both PTSD and substance use. This model follows the same graphical representations for standard SEM already mentioned. In addition, the small double headed curved arrows linking an unobserved variable to itself represent variances. The triangle is used as a placeholder to accommodate estimation of means (see Table 4 for parameter definitions).

As can be seen in this model, an observed variable PTSD severity score, $P$, corresponds to each assessment time point and is comprised of a true score $(p)$ and error $\left(e_{p}\right)$. Similarly, an 


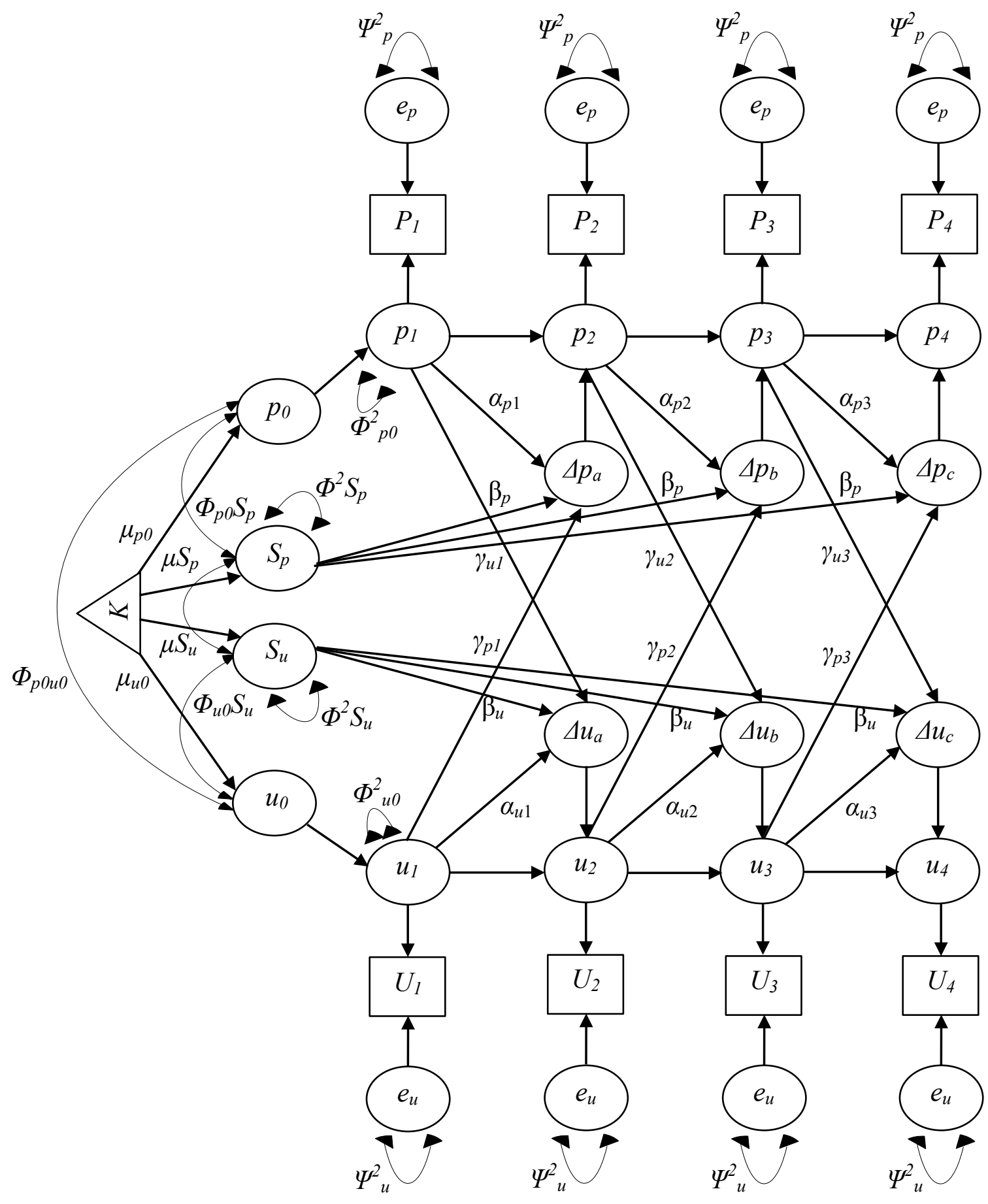

Figure 4. Example bivariate latent difference score model with four time points. 
Table 4

Bivariate Latent Difference Score Structural Equation Model Parameters.

\begin{tabular}{|c|c|}
\hline Parameter & Definition \\
\hline$K$ & Constant, deigned to estimate means and intercepts \\
\hline \multicolumn{2}{|c|}{ PTSD severity } \\
\hline$P_{1-4}$ & Observed variable PTSD severity as measured by the CAPS \\
\hline$p_{1-4}$ & Latent true score for PTSD severity \\
\hline$e_{P}$ & Latent measurement error associated with PTSD severity \\
\hline$\Delta p_{a-c}$ & PTSD severity latent difference score \\
\hline \multirow[t]{3}{*}{$\alpha_{p 1-3}$} & Proportional change coefficients for PTSD severity (i.e., autoregressive effect \\
\hline & of prior true score status of PTSD severity on PTSD severity latent difference \\
\hline & score) \\
\hline \multirow[t]{2}{*}{$S_{p}$} & Unobserved nonstationarity for PTSD severity (i.e., natural change in PTSD \\
\hline & severity over time) \\
\hline \multirow[t]{3}{*}{$\beta_{p}$} & Effect of PTSD severity nonstationarity on PTSD severity latent difference \\
\hline & score (i.e., effect of natural change in PTSD severity over time on PTSD \\
\hline & severity latent difference score) \\
\hline$\mu_{p 0}$ & Mean of initial true PTSD severity score \\
\hline$\mu S_{p}$ & Mean of PTSD nonstationarity \\
\hline$\Psi_{p}^{2}$ & Variance associated with PTSD severity measurement error \\
\hline$\Phi_{p 0}^{2}$ & Variance of initial true PTSD severity score \\
\hline$\Phi^{2} S_{p}$ & Variance of PTSD nonstationarity \\
\hline$\Phi_{p 0} S_{p}$ & Covariance between true PTSD severity true score and nonstationarity \\
\hline
\end{tabular}




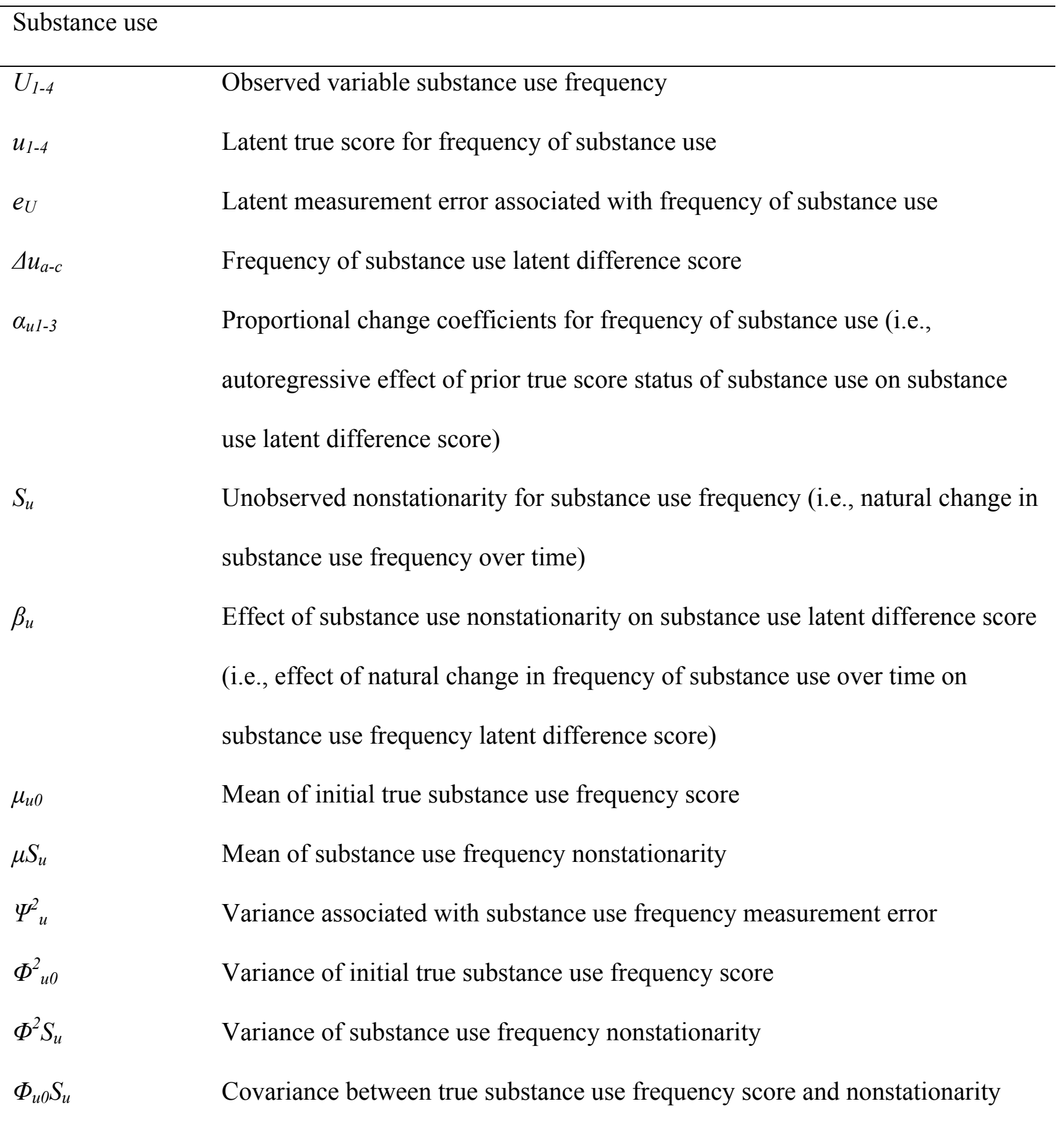

\section{Relationship between PTSD and substance use}

$\gamma_{u 1-3}$

Cross-lagged influence of true PTSD severity on substance use frequency latent difference score (i.e., influence of PTSD severity at the prior time point on change in substance use frequency at the subsequent time point) 
$\gamma_{p 1-3} \quad$ Cross-lagged influence of true substance use frequency on PTSD severity latent difference score (i.e., influence of substance use frequency at the prior time point on change in PTSD severity at the subsequent time point)

$\Phi_{p 0 u 0} \quad$ Covariance between initial true PTSD severity score and initial true substance use frequency score

Note. $\mathrm{PTSD}=$ posttraumatic stress disorder. 
observed variable substance use frequency score, $U$, corresponds to each assessment time point and is comprised of a true score $(u)$ and error $\left(e_{u}\right)$. Latent difference scores for PTSD severity and substance use are denoted by $\Delta p$ and $\Delta u$, respectively. These difference scores have been identified as optimally reliable because they are calculated from differences between adjacent perfectly reliable true scores (King et al., 2009; Williams \& Zimmerman, 1996).

The proportional change coefficients (or autoregressive effects) of prior true score status on the latent difference score are represented by $\alpha$ s. These can be left to vary over time, though they are often constrained to be equal (e.g., King et al., 2009; Orth, Berking, Walker, Meier, \& Znoj, 2008). $S_{p}$ and $S_{u}$ denote the unobserved variable representing nonstationarity for PTSD and substance use, respectively, while the betas, $\beta \mathrm{s}$, symbolize the effect of nonstationarity on the latent difference scores. These $\beta$ s are typically constrained to 1 , while residual measurement error variances $\left(\Psi^{2} \mathrm{~s}\right)$ are constrained to be equal within constructs. For this dissertation, $\beta$ s were constrained to 1 , while $\alpha$ s were either constrained to be equal or permitted to vary across time as determined by the univariate LDS model used for each outcome variable based on model-to-data fit (described above). Estimation of initial status means $\left(\mu_{p 0}\right.$ and $\left.\mu_{u 0}\right)$ and variances $\left(\Phi_{p 0}^{2}\right.$ and $\left.\Phi^{2}{ }_{u 0}\right)$ for each construct, as well as the means $\left(\mu S_{p}\right.$ and $\left.\mu S_{u}\right)$ and variances $\left(\Phi^{2} S_{p}\right.$ and $\left.\Phi^{2} S_{u}\right)$ for nonstationarity for each construct are also contained within the structural model.

Extracting from the model, we see that change in PTSD severity is a function of prior substance use status, controlling for the influence of prior PTSD status (autoregressive effect) and nonstationarity (natural change) in PTSD

$$
\begin{aligned}
& \Delta p_{x}=\text { prior substance use status }+ \text { autoregressive effect }+ \text { nonstationarity } \\
& \Delta p_{x}=\gamma_{p t} u_{t}+\alpha_{p t} p_{t}+\beta_{p} S_{p}
\end{aligned}
$$


For instance, $\Delta p_{a}=\gamma_{p l} u_{1}+\alpha_{p l} p_{1}+\beta_{p} S_{p}$. The regression coefficient $\gamma_{p t}$ is the cross-lagged influence of substance use on change in PTSD severity. In this way, change in PTSD severity is a time-dependent function of itself and substance use. Similarly, the equation for dynamic change in substance use is

$$
\Delta u_{x}=\gamma_{u t} p_{t}+\alpha_{u t} u_{t}+\beta_{u} S_{u}
$$

[see King, King, McArdle, Shalev, \& Doron-LaMarca (2009), as well as McArdle \& Hamagami (2001) and McArdle (2001) for a more in-depth explanation of LDS modeling].

BLDS models for PTSD and substance use: Hypothesis $3 b$. For this study, initial BLDS models were constructed using the best fitting univariate LDS models to examine temporal relations between PTSD severity and substance use. This corresponded to Hypothesis $3 \mathrm{~b}$ and resulted in fitting 10 BLDS models to the data: (1) PTSD total symptom severity with alcohol use, (2) PTSD intrusion symptoms with alcohol use, (3) PTSD hyperarousal symptoms with alcohol use, (4) PTSD avoidance symptoms with alcohol use, (5) PTSD negative shifts in cognitions and mood symptoms with alcohol use, (6) PTSD total symptom severity with drug use, (7) PTSD intrusion symptoms with drug use, (8) PTSD hyperarousal symptoms with drug use, (9) PTSD avoidance symptoms with drug use, and (10) PTSD negative shifts in cognitions and mood symptoms with drug use.

Self-medication versus susceptibility theory models: Hypothesis $3 c$. To examine whether models representing the susceptibility theory resulted in a statistically poorer fit to the data as compared to models representing the self-medication theory (Hypothesis 3c), two BLDS models reflecting these theories were constructed and compared for each of the abovementioned 10 outcome variable model combinations. Specifically, self-medication models were constructed by estimating the cross-lagged coupling effects from PTSD to substance use while constraining to 0 
the cross-lagged coupling effects from substance use to PTSD. In contrast, the cross-lagged coupling effects from PTSD to substance use were constrained to 0 and the cross-lagged coupling effects from substance use to PTSD were estimated in the susceptibility models.

\section{Self-medication versus mutual maintenance theory models: Hypothesis $3 d$.}

Additionally, BLDS models reflecting the mutual maintenance theory were constructed for each of the abovementioned 10 outcome variable model combinations to examine whether models representing the mutual maintenance theory resulted in a statistically poorer fit to the data as compared to models representing the self-medication theory (Hypothesis 3d). The mutual maintenance models were constructed by estimating the cross-lagged coupling effects from PTSD to substance use, as well as those from substance use to PTSD.

Determination of model fit. To determine adequacy of the models in representing the data established critical values of several goodness-of-fit indicators were estimated. These goodness-of-fit indices were chosen in consideration of relative strengths and weaknesses of

each index and to reduce measurement bias. Indices included model chi-square statistic $\left(\chi^{2}\right)$, normed chi square statistic $\left(\chi^{2} / \mathrm{df}\right)$, comparative fit index (CFI), root mean square error of approximation (RMSEA), and standardized root mean square residual (SRMR).

Absolute model fit was assessed with the chi-square index. The $\chi^{2}$ provides a test of the null hypothesis that differences between the covariance matrix of the proposed model and the sample covariance matrix are small enough to be presumed to be due to sampling error. The chisquare index is thus considered a "badness of fit" statistic because a failure to reject the null hypothesis is indicative of good model fit (Kline, 2011); therefore, the probability associated with the chi-square statistic should be relatively large $(p>.05)$ to demonstrate a good-fitting model (Bollen, 1989; Byrne, 2010). Although the chi-square is easily interpretable, it is sensitive 
to sample size and the number of variables within the model. Therefore, it is typical to report additional measures of model fit (Tabachnick \& Fidell, 2007). One such fit measure that is less sensitive to sample size is the normed chi-square. This value refers to the ratio of $\chi^{2}$ to degrees of freedom, where a value $<2$ is indicative of a close fit between the data and the hypothesized model (Tabachnick \& Fidell, 2007).

The CFI is an approximate fit index derived from a comparison of the hypothesized model with the independence model (the model that corresponds to unrelated variables), which takes sample size into account (Bentler, 1990; Byrne, 2010). The CFI measures the improvement in fit of the proposed model relative to the independence model (i.e., the model with no correlations among variables), with values greater than or equal to .95 indicative of good model fit. It is recommended that studies use the CFI in conjunction with the SRMR (Hu \& Bentler, 1999). The SRMR criterion represents the average differences between the observed and predicted correlations, derived from transforming the variance and covariance matrices into correlation matrices (Kline, 2011). Values less than .08 indicate a satisfactory fit, with lower values desired (Hu \& Bentler, 1999).

The RMSEA has been recognized as one of the most informative model fit indices because it takes both the number of estimated parameters and the error of approximation into account (Byrne, 2010). The precision of the RMSEA was estimated with its $90 \%$ confidence interval (CI) and significance test for closeness of fit (PCLOSE). RMSEA values of .05 or less signify a good fit between the data and the hypothesized model, while values up to 0.08 indicate a reasonable fit (Browne \& Cudeck, 1993). Standard interpretation of the PCLOSE dictates values greater than 0.05 indicate close model fit, though it has been recommended that the test of close fit should be greater than .50 (Jöreskog \& Sörbom, 1996). 
With respect to comparisons between models, the chi-squared difference test $\left(\chi^{2} \Delta\right)$ was calculated for nested models to determine if there was a decrement in model fit as freely estimated parameters in the model were eliminated. A significant chi-squared difference test indicates that the more complex model (i.e., model with more estimated parameters) is a better fit to the data. In the case of a non-significant chi-square difference test, it is recommended to use the more parsimonious model (Kline, 2011). For non-nested models, the Akaike Information Criterion (AIC) was used. This parsimony-adjusted index is used to select among competing models with smaller values indicative of a better quality model (Kline, 2011).

\section{Results}

Means $(M)$ and standard deviations $(S D)$ for PTSD total scores, PTSD symptom cluster scores, and substance use for each assessment point are displayed in Table 5. Bivariate correlations for study variables are presented in Table 6. Notably, there were few significant associations between PTSD and substance use variables, and significant correlations were of small magnitude. In contrast, all correlations between PTSD total and cluster score variables were significant and of moderate-to-large effect. Most correlations between alcohol and drug use frequency variables were also significant, with effect sizes ranging from small to large.

\section{Growth Curve Models: Aims 1 and 2}

Growth curve models for Aims 1 and 2 required participants to have completed their Time 4 assessment in order to determine whether they met criteria for end-state PTSD and/or SUD. Due to attrition, 101 of the 137 participants had completed their last assessment and, thus, were retained for analyses pertaining to the first two aims. Of these individuals, 10 had a diagnosis of PTSD-only at end-state, 14 had a diagnosis of SUD-only, 2 had a diagnosis of PTSD/SUD, and 75 participants had neither diagnosis at end-state. 
Table 5

Means and Standard Deviations for Variables of Interest Across Each of the Four Assessment Time Points.

\begin{tabular}{|c|c|c|c|c|c|c|c|c|c|c|c|c|}
\hline \multirow[b]{2}{*}{ Variable } & \multicolumn{3}{|c|}{ First Assessment } & \multicolumn{3}{|c|}{ Second Assessment } & \multicolumn{3}{|c|}{ Third Assessment } & \multicolumn{3}{|c|}{ Fourth Assessment } \\
\hline & $n$ & $M$ & $S D$ & $n$ & $M$ & $S D$ & $n$ & $M$ & $S D$ & $n$ & $M$ & $S D$ \\
\hline \multicolumn{13}{|l|}{ PTSD } \\
\hline Total & 136 & 53.10 & 0.98 & 116 & 37.65 & 32.03 & 108 & 26.83 & 29.03 & 101 & 22.07 & 29.06 \\
\hline Intrusion & 136 & 13.80 & 8.47 & 116 & 10.23 & 9.14 & 108 & 7.34 & 8.07 & 101 & 5.72 & 7.93 \\
\hline Hyperarousal & 136 & 15.39 & 9.74 & 116 & 11.00 & 9.79 & 108 & 7.57 & 8.70 & 101 & 6.37 & 8.93 \\
\hline Avoidance & 136 & 7.15 & 4.61 & 116 & 5.38 & 4.61 & 108 & 4.49 & 4.92 & 101 & 2.74 & 4.01 \\
\hline Negative Shifts & 136 & 16.76 & 12.91 & 116 & 11.03 & 12.37 & 108 & 7.43 & 10.88 & 101 & 7.24 & 11.33 \\
\hline Alcohol Use & 123 & 10.39 & 23.85 & 106 & 8.31 & 15.73 & 103 & 10.94 & 38.37 & 95 & 10.36 & 30.98 \\
\hline Drug Use & 131 & 1.79 & 4.21 & 113 & 1.62 & 4.05 & 106 & 1.45 & 3.34 & 98 & 1.22 & 2.64 \\
\hline
\end{tabular}

Note. PTSD = posttraumatic stress disorder; Negative Shifts = negative shifts in cognitions and mood. 
Table 6

Pearson Correlation Matrix for Variables at Each Assessment Point for the Total Sample

\begin{tabular}{|c|c|c|c|c|c|c|c|c|c|c|c|c|c|c|}
\hline Variable & 1. & 2. & 3. & 4. & 5. & 6. & 7. & 8. & 9. & 10. & 11. & 12. & 13. & 14. \\
\hline 1. t1 PTSD Total & -- & & & & & & & & & & & & & \\
\hline 2. t2 PTSD Total & $.76^{* *}$ & -- & & & & & & & & & & & & \\
\hline 3. t3 PTSD Total & $.65 * *$ & $.81 * *$ & -- & & & & & & & & & & & \\
\hline 4. t4 PTSD Total & $.64 * *$ & $.81 * *$ & $.83 * *$ & -- & & & & & & & & & & \\
\hline 5. t1 PTSD Intrusions & $.84 * *$ & $.70 * *$ & $.62 * *$ & $.63 * *$ & -- & & & & & & & & & \\
\hline 6. t2 PTSD Intrusions & $.66^{* *}$ & $.89 * *$ & $.71 * *$ & $.71 * *$ & $.75^{* *}$ & -- & & & & & & & & \\
\hline 7. t3 PTSD Intrusions & $.52 * *$ & $.72 * *$ & $.84 * *$ & $.75^{* *}$ & $.59 * *$ & $.72 * *$ & -- & & & & & & & \\
\hline 8. t4 PTSD Intrusions & $.56^{* *}$ & $.76^{* *}$ & $.77 * *$ & $.89 * *$ & $.63 * *$ & $.75 * *$ & $.82 * *$ & -- & & & & & & \\
\hline 9. t1 PTSD Hyperarousal & $.89 * *$ & $.64 * *$ & $.58 * *$ & $.53 * *$ & $.66^{* *}$ & $.51^{* *}$ & $.43 * *$ & $.38 * *$ & -- & & & & & \\
\hline 10. t2 PTSD Hyperarousal & $.70^{* *}$ & $.91 * *$ & $.77 * *$ & $.77^{* *}$ & $.61 * *$ & $.75^{* *}$ & $.60 * *$ & $.68 * *$ & $.65^{* *}$ & -- & & & & \\
\hline 11. t3 PTSD Hyperarousal & $.60^{* *}$ & $.76^{* *}$ & $.94^{* *}$ & $.78^{* *}$ & $.59^{* *}$ & $.68^{* *}$ & $.72 * *$ & $.71 * *$ & $.54^{* *}$ & $.76^{* *}$ & -- & & & \\
\hline 12. t4 PTSD Hyperarousal & $.59^{* *}$ & $.72 * *$ & $.78^{* *}$ & $.93^{* *}$ & $.56^{* *}$ & $.61^{* *}$ & $.66^{* *}$ & $.76^{* *}$ & $.55^{* *}$ & $.73^{* *}$ & $.78^{* *}$ & -- & & \\
\hline 13. t1 PTSD Avoidance & $.77^{* *}$ & $.51^{* *}$ & $.47 * *$ & $.37^{* *}$ & $.69^{* *}$ & $.47^{* *}$ & $.38^{* *}$ & $.35^{* *}$ & $.62 * *$ & $.45^{* *}$ & $.44 * *$ & $.33^{*}$ & -- & \\
\hline 14. t2 PTSD Avoidance & $.63 * *$ & $.80 * *$ & $.56^{* *}$ & $.51^{* *}$ & $.61^{* *}$ & $.74 * *$ & $.52 * *$ & $.50 * *$ & $.52 * *$ & $.67 * *$ & $.53^{* *}$ & $.44^{* *}$ & $.56^{* *}$ & -- \\
\hline
\end{tabular}




\begin{tabular}{|c|c|c|c|c|c|c|c|c|c|c|c|c|c|c|}
\hline Variable & 1. & 2. & 3. & 4. & 5. & 6. & 7. & 8. & 9. & 10. & 11. & 12. & 13. & 14. \\
\hline 15. t3 PTSD Avoidance & $.58^{* *}$ & $.71 * *$ & $.84 * *$ & $.60 * *$ & $.51 * *$ & $.58^{* *}$ & $.67 * *$ & $.53^{* *}$ & $.52 * *$ & $.66^{*}$ & $.76^{* *}$ & $.57 * *$ & $.53 * *$ & $.53 * *$ \\
\hline 16. t4 PTSD Avoidance & $.54 * *$ & $.69 * *$ & $.72 * *$ & $.80 * *$ & $.55^{* *}$ & $.58 * *$ & $.66^{* *}$ & $.73 * *$ & $.43^{* *}$ & $.66^{* *}$ & $.71 * *$ & $.71 * *$ & $.39 * *$ & $.54 * *$ \\
\hline 17. t1 PTSD Negative Shifts & $.90^{* *}$ & $.68 * *$ & $.55^{* *}$ & $.62 * *$ & $.62 * *$ & $.55^{* *}$ & $.40 * *$ & $.53 * *$ & $.73 * *$ & $.64 * *$ & $.49 * *$ & $.53 * *$ & $.57^{* *}$ & $.51 * *$ \\
\hline 18. t2 PTSD Negative Shifts & $.68 * *$ & $.91 * *$ & $.77 * *$ & $.76^{* *}$ & $.56^{* *}$ & $.70 * *$ & $.67 * *$ & $.68^{* *}$ & $.59 * *$ & $.77 * *$ & $.67 * *$ & $.66^{* *}$ & $.40 * *$ & $.63 * *$ \\
\hline 19. t3 PTSD Negative Shifts & $.60 * *$ & $.73 * *$ & $.91 * *$ & $.78 * *$ & $.50 * *$ & $.57 * *$ & $.63 * *$ & $.66^{* *}$ & $.56^{* *}$ & $.71 * *$ & $.82 * *$ & $.74 * *$ & $.38 * *$ & $.69 * *$ \\
\hline 20. t4 PTSD Negative Shifts & $.61^{* *}$ & $.73 * *$ & $.71 * *$ & $.93 * *$ & $.54 * *$ & $.61^{* *}$ & $.60 * *$ & $.72 * *$ & $.50^{* *}$ & $.69 * *$ & $.64 * *$ & $.82 * *$ & $.32 * *$ & $.49 * *$ \\
\hline 21. t1 Alcohol Use & .13 & .11 & .09 & .11 & .10 & .05 & $.21^{*}$ & .08 & .13 & .08 & .05 & .11 & $.23 *$ & .15 \\
\hline 22. t2 Alcohol Use & .10 & -.01 & .00 & .04 & .11 & -.02 & .06 & -.01 & .08 & -.02 & -.06 & .02 & $.21 *$ & .06 \\
\hline 23. t3 Alcohol Use & .04 & -.04 & -.05 & -.04 & .02 & -.10 & -.05 & -.07 & .11 & -.01 & -.08 & .00 & .14 & .02 \\
\hline 24. t4 Alcohol Use & .05 & .05 & -.00 & .04 & .00 & -.05 & -.04 & -.03 & .14 & .06 & -.03 & .09 & .07 & .04 \\
\hline 25. t1 Drug Use & .08 & .09 & -.04 & -.02 & .00 & -.00 & -.14 & -.08 & .06 & .13 & -.06 & -.05 & .10 & .12 \\
\hline 26. t2 Drug Use & .02 & .15 & .06 & -.00 & .00 & .05 & .09 & -.08 & .04 & .17 & .01 & -.02 & -.01 & .17 \\
\hline 27. t3 Drug Use & .10 & .06 & -.03 & .00 & .07 & .00 & -.12 & -.06 & .08 & .06 & -.05 & -.02 & $.21 *$ & .09 \\
\hline 28. t4 Drug Use & .20 & .08 & .07 & .11 & .15 & -.02 & -.03 & .00 & $.23 *$ & .11 & .07 & .10 & $.20^{*}$ & .11 \\
\hline
\end{tabular}




\begin{tabular}{|c|c|c|c|c|c|c|c|c|c|c|c|c|c|c|}
\hline Variable & 15. & 16. & 17. & 18. & 19. & 20. & 21. & 22. & 23. & 24. & 25. & 26. & 27. & 28 . \\
\hline 15. t3 PTSD Avoidance & -- & & & & & & & & & & & & & \\
\hline 16. t4 PTSD Avoidance & $.63 * *$ & -- & & & & & & & & & & & & \\
\hline 17. t1 PTSD Negative Shifts & $.47 * *$ & $.47 * *$ & -- & & & & & & & & & & & \\
\hline 18. t2 PTSD Negative Shifts & $.68^{* *}$ & $.63 * *$ & $.67 * *$ & -- & & & & & & & & & & \\
\hline 19. t3 PTSD Negative Shifts & $.69 * *$ & $.59 * *$ & $.56^{* *}$ & $.73 * *$ & -- & & & & & & & & & \\
\hline 20. t4 PTSD Negative Shifts & $.49 * *$ & $.63^{* *}$ & $.63^{* *}$ & $.73 * *$ & $.75^{* *}$ & -- & & & & & & & & \\
\hline 21. t1 Alcohol Use & $.21^{*}$ & $.23^{*}$ & .06 & .13 & -.04 & .06 & -- & & & & & & & \\
\hline 22. t2 Alcohol Use & .10 & .06 & .03 & -.01 & -.03 & .08 & $.53 * *$ & -- & & & & & & \\
\hline 23. t3 Alcohol Use & .00 & -.07 & -.06 & -.03 & -.02 & -.04 & $.33^{*}$ & $.45^{* *}$ & -- & & & & & \\
\hline 24. t4 Alcohol Use & -.01 & -.03 & -.01 & .09 & .04 & .07 & $.36^{*}$ & $.38^{* *}$ & $.91 * *$ & -- & & & & \\
\hline 25. t1 Drug Use & .14 & .10 & .08 & .08 & -.03 & .00 & .11 & $.22 *$ & $.20 *$ & .13 & -- & & & \\
\hline 26. t2 Drug Use & $.24 *$ & .10 & .15 & .15 & -.02 & .03 & .13 & $.25 *$ & $.27 *$ & $.26^{*}$ & $.76^{* *}$ & -- & & \\
\hline 27. t3 Drug Use & .16 & .07 & .08 & .08 & -.02 & .04 & $.22 *$ & $.45^{* *}$ & $.30^{*}$ & $.31 *$ & $.84 * *$ & $.57 * *$ & -- & \\
\hline 28. t4 Drug Use & .20 & .18 & .10 & .10 & .08 & .12 & .19 & $.37 * *$ & $.33 *$ & $.24 *$ & $.79 * *$ & $.76 * *$ & $.67 * *$ & -- \\
\hline
\end{tabular}

*correlation is significant at the .05 level

** correlation is significant at the .001 level.

Note. $\mathrm{PTSD}=$ posttraumatic stress disorder; Negative Shifts $=$ negative shifts in cognitions and mood. 
Results of the growth curve model with time as a predictor of PTSD total symptom severity indicated that, on average, participants had an initial PTSD total severity score of 57.97 that was significantly different than $0, S E=3.42, t(100)=16.97, p<.001, d=3.39$. Additionally, PTSD total severity significantly decreased over time by 0.07 points per day, $b=$ $0.07, S E=0.01, t(100)=-11.75, p<.001, d=-2.35$. When entered into the model at Level-2, end-state PTSD diagnosis was significantly associated with initial PTSD symptom severity (i.e., intercept); individuals with PTSD at the final assessment, on average, had an initial PTSD total severity score that was 30 points higher than individuals without end-state PTSD. Furthermore, there was a significant association between end-state PTSD diagnosis and the trajectory of PTSD total symptom severity (i.e., slope) such that individuals without PTSD at end-state had a steeper decline in PTSD symptom severity posttrauma as compared to those with a PTSD diagnosis at the final assessment. In contrast, there was no significant association between end-state SUD diagnosis and the trajectory of PTSD symptom severity. Similarly, the association between endstate SUD diagnosis and initial PTSD total score was also nonsignificant, though Cohen's $d$ indicated a small positive effect size (Table 7 and Figure 5).

With respect to the model examining time as a predictor of alcohol consumption, results suggested that individuals initially consumed an average of approximately 10 alcoholic beverages over the course of a month, $b=10.20, S E=2.76, t(100)=3.69, p<.001, d=0.74$, though the number of drinks consumed did not significantly change over time, $b<0.01, S E=$ $0.01, t(100)=0.08, p=.937, d=0.02$. When end-state PTSD diagnosis was entered into the model at Level-2, there was no significant association between initial alcohol consumption or the trajectory of alcohol consumption. Similarly, end-state SUD was not significantly associated with change in alcohol use over time (Table 8 and Figure 5). While the association between end- 
Table 7

Growth Curve Models Predicting Initial PTSD Total Symptom Severity and Symptom Trajectory from End-State Diagnoses.

\begin{tabular}{lcccccc}
\hline Effect & $b$ & $S E$ & $t$ & $d f$ & $p$ & $d$ \\
\hline Initial PTSD Symptom Severity (intercept) & 53.86 & 3.98 & 13.52 & 98 & $<.001$ & 2.73 \\
End-State PTSD Diagnosis & 30.31 & 11.38 & 2.67 & 98 & .009 & 0.54 \\
End-State SUD Diagnosis & 7.39 & 7.03 & 1.05 & 98 & .296 & 0.21 \\
PTSD Symptom Severity Trajectory (slope) & -0.08 & 0.01 & -11.56 & 98 & $<.001$ & -2.34 \\
$\quad$ & & & & & & \\
$\quad$ End-State PTSD Diagnosis & 0.09 & 0.02 & 5.58 & 98 & $<.001$ & 1.13 \\
$\quad$ & & & & & & \\
End-State SUD Diagnosis & -0.01 & 0.01 & -0.47 & 98 & .640 & -0.09
\end{tabular}

Note. The end-state PTSD diagnostic variable was dummy coded with "no PTSD diagnosis" as the comparison and the end-state SUD diagnostic variable was dummy coded with "no SUD diagnosis" as the comparison; these variables were entered as uncentered. PTSD = posttraumatic stress disorder; SUD = substance use disorder; End-state PTSD = PTSD diagnostic status based on meeting cluster criteria on the Clinician Administered PTSD Scale at the final assessment; End-state SUD = SUD diagnostic status based on meeting DSM-5 criteria as based on the MINI International Neuropsychiatric Interview at the final assessment. 

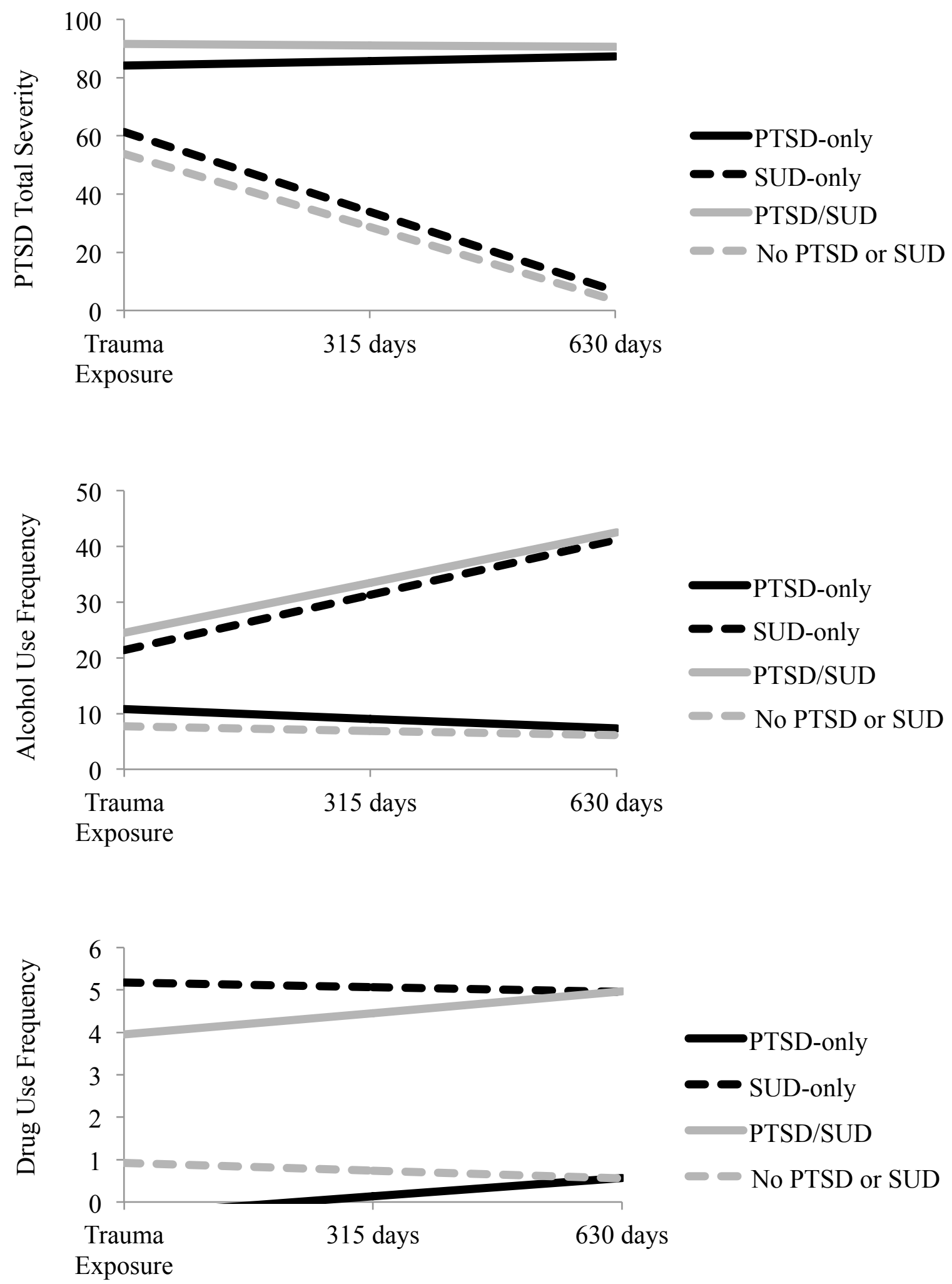

Figure 5. Trajectories of PTSD total symptom severity, alcohol use frequency, and drug use 
frequency based on end-state PTSD and/or SUD diagnostic status.

Note. End-State PTSD = PTSD diagnosis at the final assessment based on the ClinicianAdministered PTSD Scale; End-State SUD = substance use disorder diagnosis at the final assessment based on the Mini International Neuropsychiatric Inventory. 
Table 8

Growth Curve Models Predicting Initial Alcohol Use Frequency and Trajectory of Alcohol Use from End-State Diagnoses.

\begin{tabular}{|c|c|c|c|c|c|c|}
\hline Effect & $b$ & $S E$ & $t$ & $d f$ & $p$ & $d$ \\
\hline Initial Alcohol Use Frequency (intercept) & 7.71 & 2.80 & 2.75 & 98 & .007 & 0.56 \\
\hline End-State PTSD Diagnosis & 3.10 & 5.59 & 0.56 & 98 & .580 & 0.11 \\
\hline End-State SUD Diagnosis & 13.69 & 11.02 & 1.24 & 98 & .217 & 0.25 \\
\hline Alcohol Use Frequency Trajectory (slope) & $<-0.01$ & 0.01 & -0.36 & 98 & .721 & -0.07 \\
\hline End-State PTSD Diagnosis & $<-0.01$ & 0.01 & -0.23 & 98 & .817 & -0.05 \\
\hline End-State SUD Diagnosis & 0.03 & 0.06 & 0.59 & 98 & .556 & 0.12 \\
\hline
\end{tabular}

Note. The end-state PTSD diagnostic variable was dummy coded with "no PTSD diagnosis" as the comparison and the end-state SUD diagnostic variable was dummy coded with "no SUD diagnosis" as the comparison; these variables were entered as uncentered. PTSD = posttraumatic stress disorder; SUD = substance use disorder; End-state PTSD = PTSD diagnostic status based on meeting cluster criteria on the Clinician Administered PTSD Scale at the final assessment; End-state SUD = SUD diagnostic status based on meeting DSM-5 criteria as based on the MINI International Neuropsychiatric Interview at the final assessment. 
state SUD and initial alcohol consumption was non-significant, it is notable that the effect size was small and positive $(d=0.25)$. See Table 8 and Figure 5 for model results.

Results of the model with time as a predictor of drug use frequency indicated that, on average, participants initially used drugs less than twice per month, $b=1.38, S E=0.35, t(100)=$ $3.90, p<.001, d=0.78$; however, frequency of drug use did not change over time, $b=<-0.01$, $S E<0.01 t(100)=-0.78, p<.436, d=-0.16$. When entered into the model at Level-2, there was a significant association between end-state PTSD diagnosis and initial drug use, such that participants with a diagnosis of PTSD at the final assessment used drugs less frequently than those without a diagnosis of PTSD. While the association between end-state PTSD diagnosis and the trajectory of drug use was nonsignificant, Cohen's $d$ suggested a small and positive effect size. End-state SUD diagnosis also had a significant effect on initial drug use, but not the trajectory of use posttrauma exposure; participants with a diagnosis of SUD had higher rates of drug use compared to participants without SUD (see Table 9 and Figure 5).

\section{Bivariate Latent Difference Score Structural Equation Models: Aim 3}

To explore temporal sequencing of PTSD symptoms and substance use, alternative time structures varying in the representation of time since traumatic event exposure were developed and compared. Table 10 displays descriptive statistics for each of the time structures. The first time structure was modeled after the study's four assessment occasions and thus has four time segments, with fewer participants at each subsequent assessment occasion due to attrition. For the 120- and 90-days-based time structures, time since trauma exposure was classified by five and six time segments, respectively. Of note, though the 120- and 90-days-based time structures resulted in additional missing data by design, there was a general trend toward less dispersion of the timing of participant scores relative to the common time of assessment assigned to each 
Table 9

Growth Curve Models Predicting Initial Drug Use Frequency and Trajectory of Drug Use from End-State Diagnoses.

\begin{tabular}{lcccccc}
\hline Effect & $b$ & $S E$ & $t$ & $d f$ & $p$ & $d$ \\
\hline Initial Drug Use Frequency (intercept) & 0.92 & 0.25 & 3.76 & 98 & $<.001$ & 0.76 \\
End-State PTSD Diagnosis & -1.22 & 0.37 & -3.28 & 98 & .001 & -0.66 \\
End-State SUD Diagnosis & 4.26 & 1.78 & 2.39 & 98 & .019 & 0.48 \\
Drug Use Frequency Trajectory (slope) & $<-0.01$ & $<0.01$ & -1.61 & 98 & .110 & -0.33 \\
End-State PTSD Diagnosis & $<0.01$ & $<0.01$ & 1.45 & 98 & .152 & 0.29 \\
$\quad$ & & & & & & \\
End-State SUD Diagnosis & $<0.01$ & $<0.01$ & 0.10 & 98 & .924 & 0.02
\end{tabular}

Note. The end-state PTSD diagnostic variable was dummy coded with "no PTSD diagnosis" as the comparison and the end-state SUD diagnostic variable was dummy coded with "no SUD diagnosis" as the comparison; these variables were entered as uncentered. PTSD = posttraumatic stress disorder; SUD = substance use disorder; End-state PTSD = PTSD diagnostic status based on meeting cluster criteria on the Clinician Administered PTSD Scale at the final assessment; End-state SUD = SUD diagnostic status based on meeting DSM-5 criteria as based on the MINI International Neuropsychiatric Interview at the final assessment. 
Table 10

Means and Standard Deviations (Based on Days Since Traumatic Event Exposure) for Time Structures

Occasions-of-Measurement-Based Structure (4 Time Segments)

Assessment $1 \quad$ Assessment $2 \quad$ Assessment $3 \quad$ Assessment 4

\begin{tabular}{lcccc}
\hline$n$ & 137 & 117 & 110 & 102 \\
Days $M$ & 127.14 & 263.62 & 387.71 & 513.12 \\
Days $S D$ & 54.83 & 59.76 & 55.32 & 55.36
\end{tabular}

Structure Based on 120-Day Intervals (5 Time Segments)

\begin{tabular}{lccccc}
\cline { 2 - 5 } & $1 \leq 120$ & $121-240$ & $241-360$ & $361-480$ & $\geq 481$ \\
\hline$n$ & 63 & 123 & 108 & 101 & 70 \\
Days $M$ & 76.63 & 183.80 & 314.77 & 428.51 & 543.69 \\
Days $S D$ & 24.89 & 33.98 & 32.10 & 35.86 & 36.26
\end{tabular}

Structure Based on 90-Day Intervals (6 Time Segments)

\begin{tabular}{lcccccc} 
& $1 \leq 90$ & $91-180$ & $181-270$ & $271-360$ & $361-450$ & $\geq 451$ \\
\hline$n$ & 41 & 78 & 80 & 95 & 83 & 89 \\
Days $M$ & 63.10 & 138.56 & 217.20 & 322.89 & 420.43 & 527.03 \\
Days $S D$ & 19.71 & 27.65 & 25.74 & 25.51 & 34.92 & 45.54 \\
\hline
\end{tabular}


segment as the number of time segments increased. The parameter estimates for growth curve models used to evaluate the three time structures are presented in Table 11; for each, PTSD total symptom severity was modeled as the outcome. While initial intercepts were comparable across time structures, the deviance estimate suggested the 120-days-based time structure demonstrated the best fit to the data. As such, the 120-days-based time structure was imposed on all univariate LDS and BLDS models to investigate relations between PTSD symptoms and substance use across time.

Univariate LDS models for outcome variables. Univariate LDS models were fit for single-indicator PTSD and substance use outcomes, with the dual variable change models used as the comparative models. For PTSD symptom cluster scores, model fit statistics are displayed in Table 12. Examination of the goodness of fit indices for PTSD intrusion and PTSD negative shifts in cognitions and mood indicated that PTSD change over time was best represented by the dual change model with proportional change freely varying (i.e., fit indices for other univariate LDS models demonstrated poor fit to the data, chi-square difference tests compared to the dual variable proportional change models were significant, and AICs were larger than the dual variable models). While the dual fixed proportional change model for the PTSD hyperarousal symptom cluster demonstrated an adequate fit to the data, constraining the proportional change component to be equivalent across time resulted in a significantly poorer fit to the data as compared to the dual variable model with time-varying proportional change, $\chi^{2} \Delta(p)=.012$. Regarding the PTSD avoidance symptom cluster, goodness of fit indices indicated adequate model-to-data fit for both the dual variable and constant change models, with a nonsignificant chi-square difference test $(p=.063)$ favouring the more parsimonious constant change model. However, due to less than optimal SRMR and RMSEA scores for the PTSD avoidance constant 
Table 11

Deviance Statistics and Parameter Estimates for the Three Growth Curve Models Varied by Time Representation

\begin{tabular}{|c|c|c|c|c|c|c|}
\hline & \multirow{2}{*}{\multicolumn{2}{|c|}{$\begin{array}{l}\text { Occasions-based } \\
\text { Time Structure }\end{array}$}} & \multirow{2}{*}{\multicolumn{2}{|c|}{$\begin{array}{l}\text { 120-days-based } \\
\text { Time Structure }\end{array}$}} & \multirow{2}{*}{\multicolumn{2}{|c|}{$\begin{array}{l}\text { 90-days-based } \\
\text { Time Structure }\end{array}$}} \\
\hline & & & & & & \\
\hline & Estimate & $S E$ & Estimate & $S E$ & Estimate & $S E$ \\
\hline Deviance & 4139.18 & & 4137.61 & & 4157.05 & \\
\hline Intercept & 61.29 & 3.03 & 65.93 & 3.32 & 65.10 & 3.26 \\
\hline Slope & -10.10 & 0.79 & -9.52 & 0.75 & -7.23 & 0.58 \\
\hline Intercept Variance $\mathrm{e}^{\mathrm{a}}$ & 934.74 & 30.57 & 1067.72 & 32.68 & 987.63 & 31.43 \\
\hline Slope Variance ${ }^{\mathrm{a}}$ & 32.07 & 5.66 & 29.62 & 5.44 & 15.71 & 3.96 \\
\hline Residual Variance $\mathrm{a}^{\mathrm{a}}$ & 189.99 & 13.78 & 187.86 & 13.71 & 201.22 & 14.19 \\
\hline
\end{tabular}

${ }^{\mathrm{a}}$ Standard deviations are presented for estimates rather than the standard error.

Note. Posttraumatic stress disorder (PTSD) total symptom severity as measured by the ClinicianAdministered PTSD Scale was used as the outcome variable in growth curve models evaluating time structures. Accordingly, participants' PTSD total severity scores were sorted into their corresponding time segments for the 120- and 90-days-based time structures. The occasionsbased time structure was based on the time of actual assessment relative to date of traumatic event exposure. Estimate $=$ parameter estimate. 
Table 12

Goodness of Fit Estimates for Univariate Latent Difference Score Models for PTSD Symptom Clusters

PTSD Intrusion Symptom Cluster Scores

\begin{tabular}{|c|c|c|c|c|c|c|c|c|c|c|}
\hline Change Models & $\chi^{2}(d f)$ & $\chi^{2} / d f$ & $p$ & CFI & RMSEA $(90 \% \mathrm{CI})$ & PCLOSE & SRMR & AIC & $\chi^{2} \Delta$ & $p$ \\
\hline Dual Variable & $14.88(10)$ & 1.49 & .136 & 0.98 & $0.06(0.00,0.12)$ & .352 & 0.06 & 2995.90 & - & - \\
\hline Dual Fixed & $29.31(13)$ & 2.25 & .006 & 0.94 & $0.10(0.05,0.14)$ & .053 & 0.14 & 3004.33 & 14.43 & .002 \\
\hline Variable Proportional & $93.52(13)$ & 7.19 & $<.001$ & 0.72 & $0.21(0.17,0.25)$ & $<.001$ & 0.29 & 3068.54 & 78.64 & $<.001$ \\
\hline Fixed Proportional & $97.46(16)$ & 6.09 & $<.001$ & 0.72 & $0.19(0.16,0.23)$ & $<.001$ & 0.30 & 3066.48 & 82.58 & $<.001$ \\
\hline Constant & $36.33(14)$ & 2.60 & $<.001$ & 0.92 & $0.11(0.07,0.15)$ & .016 & 0.15 & 3009.35 & 21.45 & $<.001$ \\
\hline No Change & $664.52(19)$ & 34.97 & $<.001$ & $<0.01$ & $0.50(0.47,0.53)$ & $<.001$ & 1.01 & 3627.54 & 649.64 & $<.001$ \\
\hline
\end{tabular}

PTSD Hyperarousal Symptom Cluster Scores

\begin{tabular}{|c|c|c|c|c|c|c|c|c|c|c|}
\hline Change Models & $\chi^{2}(d f)$ & $\chi^{2} / d f$ & $p$ & CFI & RMSEA $(90 \% \mathrm{CI})$ & PCLOSE & SRMR & AIC & $\chi^{2} \Delta$ & $p$ \\
\hline Dual Variable & $9.34(10)$ & 0.93 & .501 & 1.00 & $<0.01(0.00,0.09)$ & .740 & 0.05 & 3140.98 & - & - \\
\hline Dual Fixed & $20.38(13)$ & 1.57 & .086 & 0.97 & $0.06(0.00,0.12)$ & .295 & 0.11 & 3146.02 & 11.04 & .012 \\
\hline Variable Proportional & $75.00(13)$ & 5.77 & $<.001$ & 0.75 & $0.19(0.15,0.23)$ & $<.001$ & 0.24 & 3200.64 & 65.66 & $<.001$ \\
\hline Fixed Proportional & $77.89(16)$ & 4.87 & $<.001$ & 0.75 & $0.17(0.13,0.21)$ & $<.001$ & 0.26 & 3197.53 & 68.55 & $<.001$ \\
\hline
\end{tabular}




\begin{tabular}{lrrrrrrrrrr} 
Constant & $25.86(14)$ & 1.85 & .027 & 0.95 & $0.08(0.03,0.13)$ & .149 & 0.12 & 3149.50 & 16.52 & .002 \\
No Change & $606.55(19)$ & 31.92 & $<.001$ & $<0.01$ & $0.48(0.44,0.51)$ & $<.001$ & 0.93 & 3720.19 & 597.21 & $<.001$ \\
\hline
\end{tabular}

PTSD Avoidance Symptom Cluster Scores

\begin{tabular}{|c|c|c|c|c|c|c|c|c|c|c|}
\hline Change Models & $\chi^{2}(d f)$ & $\chi^{2} / d f$ & $p$ & CFI & RMSEA $(90 \% \mathrm{CI})$ & PCLOSE & SRMR & $\mathrm{AIC}$ & $\chi^{2} \Delta$ & $p$ \\
\hline Dual Variable & $12.26(10)$ & 1.23 & .268 & 0.99 & $0.04(0.00,0.11)$ & .526 & 0.05 & 2575.05 & - & - \\
\hline Dual Fixed & $20.93(13)$ & 1.61 & .074 & 0.95 & $0.07(0.00,0.12)$ & .270 & 0.11 & 2577.73 & 8.67 & .034 \\
\hline Variable Proportional & $48.73(13)$ & 3.75 & $<.001$ & 0.76 & $0.14(0.10,0.19)$ & $<.001$ & 0.22 & 2605.52 & 36.47 & $<.001$ \\
\hline Fixed Proportional & $52.68(16)$ & 3.29 & $<.001$ & 0.75 & $0.13(0.09,0.17)$ & .001 & 0.23 & 2603.48 & 40.42 & $<.001$ \\
\hline Constant & $21.17(14)$ & 1.51 & .097 & 0.95 & $0.06(0.00,0.11)$ & .327 & 0.11 & 2575.97 & 8.91 & .063 \\
\hline No Change & $462.00(19)$ & 24.32 & $<.001$ & $<0.01$ & $0.41(0.38,0.45)$ & $<.001$ & 0.81 & 3006.80 & 449.74 & $<.001$ \\
\hline
\end{tabular}

PTSD Negative Shifts in Cognitions and Mood Symptom Cluster Scores

\begin{tabular}{lccccccccccc} 
Change Models & $\chi^{2}(d f)$ & $\chi^{2} / d f$ & $p$ & CFI & RMSEA (90\% CI) & PCLOSE & SRMR & AIC & $\chi^{2} \Delta$ & $p$ \\
\hline Dual Variable & $13.72(10)$ & 1.37 & .186 & 0.99 & $0.05(0.00,0.11)$ & .425 & 0.04 & 3363.27 & - & - \\
Dual Fixed & $28.74(13)$ & 2.21 & .007 & 0.94 & $0.09(0.05,0.14)$ & .060 & 0.11 & 3372.29 & 15.02 & .002 \\
Variable Proportional & $60.90(13)$ & 4.68 & $<.001$ & 0.81 & $0.16(0.12,0.21)$ & $<.001$ & 0.19 & 3404.45 & 47.18 & $<.001$ \\
Fixed Proportional & $66.87(16)$ & 4.18 & $<.001$ & 0.80 & $0.15(0.12,0.19)$ & $<.001$ & 0.21 & 3404.41 & $53.15<<.001$
\end{tabular}




$\begin{array}{lrrrrrrrrrr}\text { Constant } & 37.58(14) & 2.68 & <.001 & 0.91 & 0.11(0.07,0.15) & .012 & 0.11 & 3379.12 & 23.86 & <.001 \\ \text { No Change } & 533.08(19) & 28.06 & <.001 & <0.01 & 0.44(0.41,0.48) & <.001 & 0.81 & 3864.62 & 519.36 & <.001\end{array}$

Note. $\mathrm{PTSD}=$ posttraumatic stress disorder; $\chi^{2}=$ Chi Square statistic; $\mathrm{CFI}=$ Comparative Fit Index; RMSEA = Root Mean Square

Error of Approximation along with 90\% confidence intervals; PCLOSE = $p$ of Close Fit; SRMR = Standardized Root Mean Square

Residual; AIC $=$ Akaike Information Criterion; $\chi^{2} \Delta=$ Chi Square difference statistic relative to the dual variable proportional change model. 
change model, the dual variable proportional change model was retained for BLDS modeling. Thus, for all PTSD cluster outcomes, change over time was best represented by the dual variable model with time-varying proportional change.

Parameter estimates for the dual variable proportional change univariate LDS models for PTSD symptom clusters are presented in Table 13. For all PTSD cluster models, the initial means were positive and significantly different than zero. Additionally, for each cluster model, all of the proportional (i.e., autoregressive) change parameters were significant and negative, indicating that higher PTSD symptom cluster severity was associated with greater subsequent decreases (or smaller increases) in PTSD symptom cluster severity. The constant slope mean (i.e., nonstationarity) was significant and positive for the intrusion, negative shifts in cognitions and mood, and hyperarousal symptom cluster models, which indicated a general trend of increased PTSD cluster severity over time when controlling for the presence of prior PTSD symptoms. The constant change component played no appreciable role in the PTSD avoidance cluster univariate LDS model.

Model fit indices for substance use outcomes are displayed in Table 14. With respect to alcohol use frequency, change in use was best represented by the dual change model with proportional change fixed to equality. While fit indices were similar between the dual variable and dual fixed proportional change models, the dual fixed change model demonstrated a nonsignificant chi-square difference test $(p=.263)$ when compared to the dual variable model; thus, the more parsimonious dual fixed change model was retained for BLDS analyses. For drug use frequency, the dual variable, dual fixed, and constant change models had covariance matrices that were not positive definite. As a result, the variable proportional change model was used as a basis of comparison. Goodness of fit indices indicated good model-to-data fit for both the 
Table 13

Parameter Estimates for Best-Fitting Univariate Latent Difference Score Models for PTSD Symptom Clusters

\begin{tabular}{|c|c|c|c|c|c|c|}
\hline & \multicolumn{3}{|c|}{ PTSD Intrusion } & \multicolumn{3}{|c|}{ PTSD Hyperarousal } \\
\hline & \multicolumn{3}{|c|}{ Dual Variable Change Model } & \multicolumn{3}{|c|}{ Dual Variable Change Model } \\
\hline & Estimate & $S E$ & $p$ & Estimate & $S E$ & $p$ \\
\hline \multicolumn{7}{|l|}{ Parameter Estimates } \\
\hline Initial status mean & 14.53 & 0.92 & $<.001$ & 15.11 & 1.08 & $<.001$ \\
\hline Constant change mean (nonstationarity) & 2.28 & 1.02 & .025 & 2.42 & 1.21 & .045 \\
\hline \multicolumn{7}{|l|}{ Proportional (autoregressive) change } \\
\hline$\alpha_{p 1}$ & -0.40 & 0.08 & $<.001$ & -0.30 & 0.09 & .001 \\
\hline$\alpha_{p 2}$ & -0.43 & 0.10 & $<.001$ & -0.50 & 0.10 & $<.001$ \\
\hline$\alpha_{p 3}$ & -0.58 & 0.11 & $<.001$ & -0.50 & 0.13 & $<.001$ \\
\hline$\alpha_{p 4}$ & -0.67 & 0.14 & $<.001$ & -0.62 & 0.14 & $<.001$ \\
\hline Error variance & 15.68 & 1.55 & $<.001$ & 21.77 & 2.18 & $<.001$ \\
\hline & \multicolumn{3}{|c|}{ PTSD Avoidance } & \multicolumn{3}{|c|}{ PTSD Negative Shifts } \\
\hline & \multicolumn{3}{|c|}{ Dual Variable Change Model } & \multicolumn{3}{|c|}{ Dual Variable Change Model } \\
\hline
\end{tabular}




\begin{tabular}{lcccccc} 
& Estimate & $S E$ & $p$ & Estimate & $S E$ & $p$ \\
\hline Parameter estimates & & & & & & \\
$\quad$ Initial status mean & 6.63 & 0.53 & $<.001$ & 17.01 & 1.39 & $<.001$ \\
Constant change mean (nonstationarity) & 0.57 & 0.70 & .411 & 3.51 & 1.54 & .022 \\
Proportional change & & & & & & \\
$\alpha_{p 1}$ & -0.25 & 0.12 & .034 & -0.38 & 0.10 & $<.001$ \\
$\alpha_{p 2}$ & -0.34 & 0.14 & .017 & -0.66 & 0.12 & $<.001$ \\
$\alpha_{p 3}$ & -0.40 & 0.17 & .017 & -0.50 & 0.17 & .003 \\
$\alpha_{p 4}$ & -0.64 & 0.18 & $<.001$ & -0.72 & 0.16 & $<.001$ \\
Error variance & 8.04 & 0.77 & $<.001$ & 37.03 & 3.63 & $<.001$
\end{tabular}

Note. PTSD = posttraumatic stress disorder; PTSD negative shifts = negative shifts in cognitions and mood; $\alpha_{p l-4}=$ proportional change parameters. 
Table 14

Goodness of Fit Estimates for Univariate Latent Difference Score Models for Substance Use Frequencies

Alcohol Use Frequency

\begin{tabular}{|c|c|c|c|c|c|c|c|c|c|c|}
\hline Change Models & $\chi^{2}(d f)$ & $\chi^{2} / d f$ & $p$ & CFI & RMSEA $(90 \%$ CI $)$ & PCLOSE & SRMR & AIC & $\chi^{2} \Delta$ & $p$ \\
\hline Dual Variable & $20.69(10)$ & 2.07 & .023 & 0.95 & $0.09(0.03,0.14)$ & .110 & 0.09 & 258.24 & - & - \\
\hline Dual Fixed & $24.68(13)$ & 1.90 & .025 & 0.95 & $0.08(0.03,0.13)$ & .134 & 0.09 & 256.24 & 3.99 & .263 \\
\hline Variable Proportional & $31.01(13)$ & 2.39 & .003 & 0.92 & $0.10(0.06,0.15)$ & .034 & 0.09 & 262.57 & 10.32 & .016 \\
\hline Fixed Proportional & $33.83(16)$ & 2.11 & .006 & 0.92 & $0.09(0.05,0.13)$ & .059 & 0.08 & 259.39 & 13.14 & .041 \\
\hline Constant & $27.13(14)$ & 1.94 & .019 & 0.94 & $0.08(0.03,0.13)$ & .114 & 0.09 & 256.69 & 6.44 & .169 \\
\hline No Change & 599.67 (19) & 31.56 & $<.001$ & $<0.01$ & $0.48(0.45,0.51)$ & $<.001$ & 0.85 & 819.23 & 578.98 & $<.001$ \\
\hline \multicolumn{11}{|c|}{ Drug Use Frequency } \\
\hline Change Models & $\chi^{2}(d f)$ & $\chi^{2} / d f$ & $p$ & CFI & RMSEA $(90 \% \mathrm{CI})$ & PCLOSE & SRMR & AIC & $\chi^{2} \Delta$ & $p$ \\
\hline Dual Variable & & \multicolumn{9}{|c|}{ Model covariance matrix was not positive definite } \\
\hline Dual Fixed & & \multicolumn{9}{|c|}{ Model covariance matrix was not positive definite } \\
\hline Variable Proportional $^{\mathrm{a}}$ & $15.38(13)$ & 1.18 & .284 & 0.99 & $0.04(0.00,0.10)$ & .580 & 0.06 & 127.70 & - & - \\
\hline Fixed Proportional & $16.85(16)$ & 1.05 & .396 & 1.00 & $0.02(0.00,0.08)$ & .715 & 0.07 & 123.17 & 1.47 & 689 \\
\hline
\end{tabular}




$\begin{array}{llllllllll}\text { No Change } & 359.99(19) & 18.95 & <.001 & <0.01 & 0.36(0.33,0.40) & <.001 & 0.53 & 460.32 & 344.61<.001\end{array}$

${ }^{\mathrm{a}}$ Variable proportional change model used as basis of comparison with other models since the dual variable, dual fixed, and constant change model covariances were not positive definite.

Note. Alcohol Use $=$ frequency of alcohol consumption in the past month; Drug Use $=$ frequency of drug use in the past month; CAPS $=$ Clinician-Administered PTSD Scale; $\chi^{2}=$ Chi Square statistic $;$ CFI = Comparative Fit Index; RMSEA = Root Mean Square Error of Approximation along with 90\% confidence intervals; PCLOSE $=p$ of Close Fit; SRMR = Standardized Root Mean Square Residual; $\mathrm{AIC}=$ Akaike Information Criterion; $\chi^{2} \Delta=$ Chi Square difference statistic relative to the dual variable proportional change model (except where otherwise indicated). 
variable proportional and fixed proportional change models, with a non-significant chi-square difference test $(p=.689)$ favouring the more parsimonious fixed proportional change model. Parameter estimates for the best-fitting univariate LDS models for substance use outcomes are presented in Table 15. In the alcohol and drug use models, initial status means were different from 0 ; however, neither constant change nor proportional change coefficients were significant for the substance use models.

Determination of the best-fitting univariate LDS model for PTSD total symptom severity involved comparison of the single- and 4-indicator PTSD total severity univariate LDS models. As presented in Table 16, none of the univariate 4-indicator PTSD total severity LDS models demonstrated a good fit to the data (i.e., $\chi^{2} / \mathrm{df}>2.00, \mathrm{CFI}<0.95$, RMSEA $>0.08$, PCLOSE $\leq$ .05 , SRMR $\geq 0.08$ ). Examination of the single-indicator univariate LDS models demonstrated that PTSD total symptom severity was best represented by the dual change model with proportional change freely varying, with all other single-indicator LDS models demonstrating a comparatively poorer fit to the data. As such, the single-indicator dual variable proportional change LDS model was retained as the best-fitting univariate LDS model; parameter estimates for this univariate LDS model for PTSD total symptom severity are presented in Table 17. Results indicated that higher PTSD total symptom severity was associated with greater subsequent decreases (or smaller increases) in PTSD total symptom severity. The initial status mean was positive and significant, and there was a general trend of increased PTSD total symptom severity over time when controlling for the presence of prior PTSD symptoms.

BLDS models for PTSD and substance use. Figure 6 depicts the path diagram for the PTSD with alcohol use frequency BLDS models, whereby the dual variable proportional change LDS model was retained for PTSD components (i.e., total and cluster scores) and the dual fixed 
Table 15

Parameter Estimates for Best-Fitting Univariate Latent Difference Score Models for Substance Use Frequencies

\begin{tabular}{|c|c|c|c|c|c|c|}
\hline & \multicolumn{3}{|c|}{ Alcohol Use Frequency } & \multicolumn{3}{|c|}{ Drug Use Frequency } \\
\hline & \multicolumn{3}{|c|}{ Dual Fixed Change Model } & \multicolumn{3}{|c|}{ Fixed Proportional Change Model } \\
\hline & Estimate & $S E$ & $p$ & Estimate & $S E$ & $p$ \\
\hline \multicolumn{7}{|l|}{ Parameter estimates } \\
\hline Initial status mean & -0.50 & 0.04 & $<.001$ & -0.22 & 0.03 & $<.001$ \\
\hline Constant change mean (nonstationarity) & -0.19 & 0.11 & .102 & - & - & - \\
\hline \multicolumn{7}{|l|}{ Proportional (autoregressive) change } \\
\hline$\alpha_{u 1}$ & -0.41 & 0.23 & .077 & -0.02 & 0.02 & .342 \\
\hline$\alpha_{u 2}$ & -0.41 & 0.23 & .077 & -0.02 & 0.02 & .342 \\
\hline$\alpha_{u 3}$ & -0.41 & 0.23 & .077 & -0.02 & 0.02 & .342 \\
\hline$\alpha_{u 4}$ & -0.41 & 0.23 & .077 & -0.02 & 0.02 & .342 \\
\hline Error variance & 0.05 & 0.01 & $<.001$ & 0.04 & $<0.01$ & $<.001$ \\
\hline
\end{tabular}

Note. $\alpha_{u l-4}=$ proportional change parameters. 
Table 16

Goodness of Fit Estimates for 4-Indicator and Single-Indicator Univariate Latent Difference Score Models for PTSD Total Severity 4-Indicator PTSD Total Symptom Severity Models

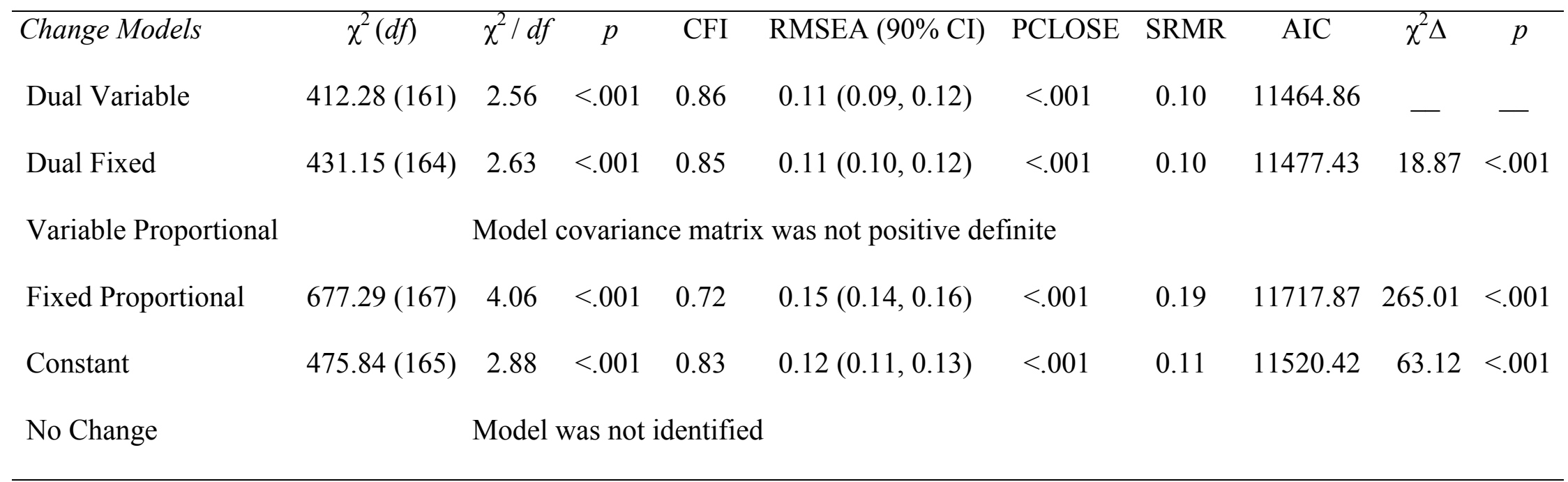

Single-Indicator PTSD Total Symptom Severity Models

\begin{tabular}{|c|c|c|c|c|c|c|c|c|c|c|}
\hline Change Models & $\chi^{2}(d f)$ & $\chi^{2} / d f$ & $p$ & CFI & RMSEA $(90 \% \mathrm{CI})$ & PCLOSE & SRMR & $\mathrm{AIC}$ & $\chi^{2} \Delta$ & $p$ \\
\hline Dual Variable & $13.18(10)$ & 1.32 & .214 & 0.99 & $0.05(0.00,0.11)$ & .461 & 0.05 & 4130.61 & - & - \\
\hline Dual Fixed & $29.02(13)$ & 2.23 & .007 & 0.95 & $0.10(0.05,0.14)$ & .056 & 0.14 & 4140.44 & 15.84 & .001 \\
\hline Variable Proportional & $105.39(13)$ & 8.11 & $<.001$ & 0.73 & $0.23(0.19,0.27)$ & $<.001$ & 0.28 & 4216.82 & 92.21 & $<.001$ \\
\hline Fixed Proportional & $107.11(16)$ & 6.69 & $<.001$ & 0.73 & $0.20(0.17,0.24)$ & $<.001$ & 0.30 & 4212.53 & 93.93 & $<.001$ \\
\hline
\end{tabular}




$\begin{array}{lrrrrrrrrrr}\text { Constant } & 40.19(14) & 2.87 & <.001 & 0.92 & 0.12(0.08,0.16) & .006 & 0.15 & 4149.61 & 27.01 & <.001 \\ \text { No Change } & 771.25(19) & 40.59 & <.001 & <0.01 & 0.54(0.51,0.57) & <.001 & 1.15 & 4870.68 & 758.07 & <.001\end{array}$

Note. $\mathrm{PTSD}=$ posttraumatic stress disorder; $\chi^{2}=$ Chi Square statistic; CFI = Comparative Fit Index; RMSEA = Root Mean Square Error of Approximation along with 90\% confidence intervals; PCLOSE = $p$ of Close Fit; SRMR = Standardized Root Mean Square Residual; AIC $=$ Akaike Information Criterion; $\chi^{2} \Delta=$ Chi Square difference statistic relative to the dual variable proportional change model. 
Table 17

Parameter Estimates for Best-Fitting Univariate Latent Difference Score Models for PTSD Total Symptom Severity

\begin{tabular}{lccc}
\hline & \multicolumn{3}{l}{ Total PTSD Symptom Severity } \\
& \multicolumn{2}{c}{ Dual Variable Change Model } \\
\cline { 2 - 4 } & Estimate & $S E$ & $p$ \\
\hline Parameter estimates & & 3.33 & $<.001$ \\
Initial status mean & 56.68 & 3.68 & $<.001$ \\
Constant change mean (nonstationarity) & 10.77 & & \\
Proportional (autoregressive) change & & 0.07 & $<.001$ \\
$\alpha_{p 1}$ & -0.37 & 0.08 & $<.001$ \\
$\alpha_{p 2}$ & -0.52 & 0.10 & $<.001$ \\
$\alpha_{p 3}$ & -0.50 & 0.11 & $<.001$ \\
$\alpha_{p 4}$ & -0.68 & 16.00 & $<.001$ \\
Error variance & & & \\
\hline Note. PTSD = posttraumatic stress disorder; $\alpha_{p 1-4}=$ proportional change parameters.
\end{tabular}




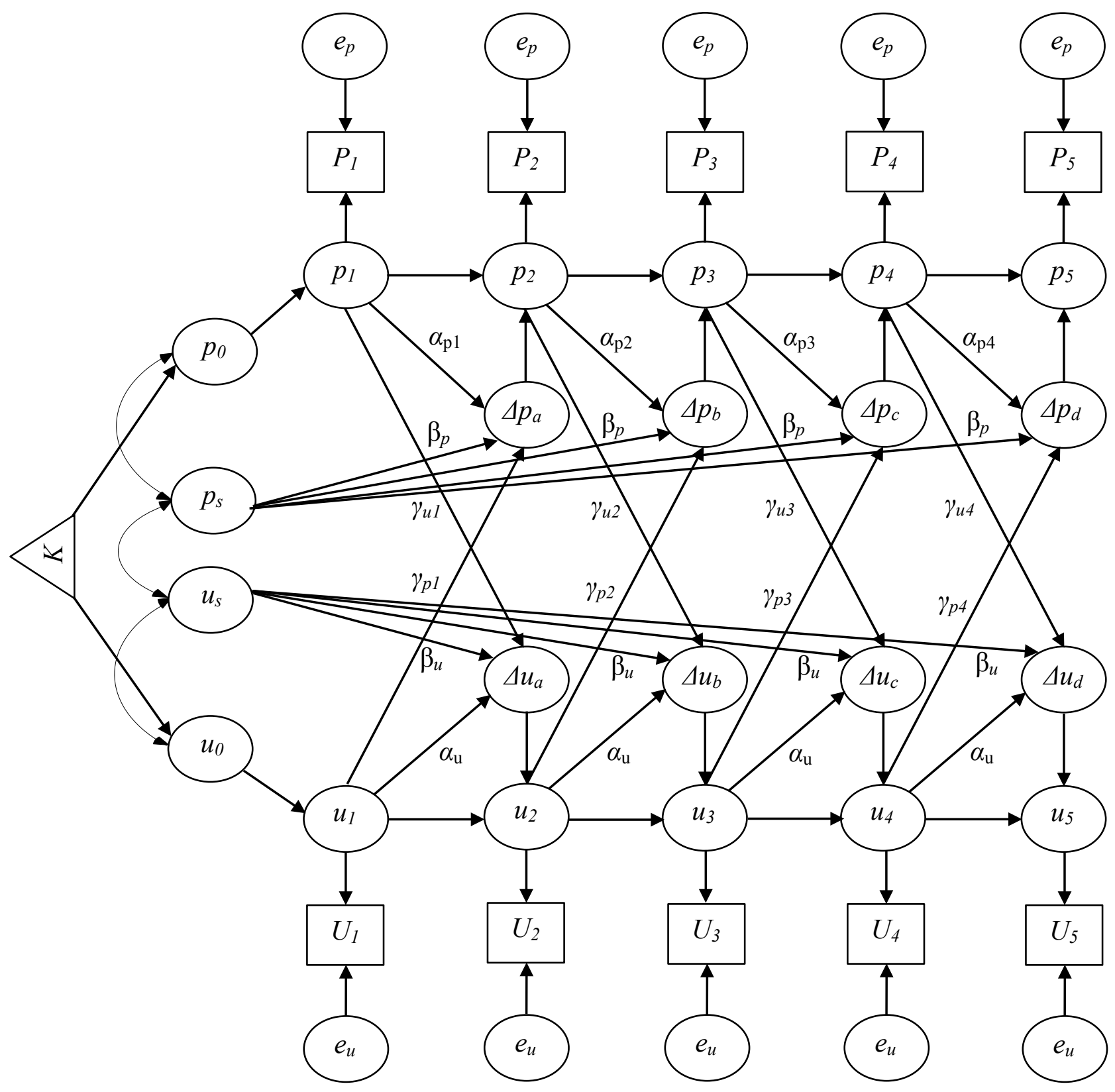

Figure 6. Bivariate latent difference score model examining associations between PTSD and alcohol use subsequent to traumatic exposure. This BLDS model was the result of pairing the best fitting univariate LDS models for PTSD components (i.e., total and PTSD clusters) and alcohol use frequency: The dual variable proportional change LDS model was retained for PTSD 
and the dual fixed proportional change LDS model was retained for alcohol use frequency. $K$ represents a constant used to estimate means and intercepts. $P_{[1-5]}$ denotes the observed PTSD scores at each time point, $p_{[1-5]}$ represents the true score latent variables for each time point, $\Delta p_{[a-}$ ${ }_{\text {d] }}$ represents the latent difference scores between corresponding time points, $p_{0}$ denotes the estimated initial status mean for PTSD, $\beta_{p}$ represents the effect of PTSD nonstationarity (i.e., constant change) on PTSD latent difference scores, $\alpha_{p[1-4]}$ represents the proportional change as a functioning of the prior true score latent variable PTSD status on the subsequent latent difference score, and $e_{\mathrm{p}}$ represents the error terms for PTSD, which were constrained to be equal across time points. $U_{[1-5]}$ denotes the observed substance use scores at each time point, $u_{[1-5]}$ represents the true score latent variables for each time point, $\Delta u_{[a-d]}$ represents the latent difference scores between corresponding time points, $u_{0}$ denotes the estimated initial status mean for substance use, $\beta_{u}$ represents the effect of substance use nonstationarity (i.e., constant change) on substance use latent difference scores, $\alpha_{u}$ represents the (fixed) proportional change as a functioning of the prior true score latent variable substance use status on the subsequent latent difference score, and $e_{\mathrm{u}}$ represents the error terms for substance use, which were constrained to be equal across time points. $\gamma_{p[1-4]}$ denotes the cross-lagged influence of true PTSD scores on substance use latent difference scores across time points, while $\gamma_{u[1-4]}$ denotes the cross-lagged influence of true substance use scores on PTSD latent difference scores across time points. 
proportional change LDS model was retained for alcohol use frequency. Each of the PTSD with alcohol use BLDS models demonstrated a good fit to the data (see Table 18). Parameter estimates for the PTSD with alcohol use BLDS models are contained in Tables 19 through 23; initial rows of each table detail the within variable portions of the BLDS model as separate for PTSD and alcohol use, while subsequent rows contain findings that relate to the linkage between PTSD and alcohol use.

There was a fairly high degree of consistency between the BLDS models for PTSD with alcohol use. In all models, the initial status means were significantly different from 0 and the constant change components for both PTSD and alcohol use played no appreciable role across time. Additionally, all proportional change parameters were significant and negative for alcohol use in the PTSD total severity, intrusion, hyperarousal, and avoidance models; thus, individuals with higher alcohol use at one time segment would be expected to use less alcohol at the subsequent time segment. All of the proportional change parameters for PTSD total and negative shifts in cognitions and mood cluster scores were significant and negative, the second through fourth proportional change paths were significant and negative for intrusion and hyperarousal clusters, and the fourth proportional change parameter for PTSD avoidance was negative and significant. This suggests that, to some extent in each of the models, higher PTSD severity was associated with greater subsequent decreases (or smaller increases) in PTSD severity. Interestingly, there were several significant cross-lagged effects. For the models involving alcohol use with PTSD intrusion, hyperarousal, and total symptoms, participants with higher PTSD scores at the second (4-8 months posttrauma) and third (8-12 months posttrauma) time intervals, on average, exhibit greater decreases (or smaller increases) in alcohol consumption over the subsequent time intervals (8-12 and 12-16 months, respectively, posttrauma). Similarly, 
Table 18

Goodness of Fit Estimates for Bivariate Latent Difference Score Models

\begin{tabular}{|c|c|c|c|c|c|c|c|}
\hline Model & $\chi^{2}(d f)$ & $\chi^{2} / d f$ & $p$ & CFI & RMSEA $(90 \% \mathrm{CI})$ & PCLOSE & SRMR \\
\hline \multicolumn{8}{|l|}{ Alcohol Use Dual Fixed Change (with): } \\
\hline PTSD Total Dual Variable Change & $53.01(36)$ & 1.47 & .034 & 0.97 & $0.06(0.02,0.09)$ & .314 & 0.06 \\
\hline PTSD Intrusion Dual Variable Change & $43.54(36)$ & 1.21 & .181 & 0.99 & $0.04(0.00,0.08)$ & .646 & 0.06 \\
\hline PTSD Hyperarousal Dual Variable Change & $55.92(36)$ & 1.55 & .018 & 0.96 & $0.06(0.03,0.10)$ & .233 & 0.07 \\
\hline PTSD Avoidance Dual Variable Change & $39.31(36)$ & 1.09 & .324 & 0.99 & $0.03(0.00,0.07)$ & .324 & 0.06 \\
\hline PTSD Negative Shifts Dual Variable Change & $61.58(36)$ & 1.71 & .005 & 0.95 & $0.07(0.04,0.10)$ & .119 & 0.07 \\
\hline \multicolumn{8}{|l|}{ Drug Use Fixed Proportional Change (with): } \\
\hline PTSD Total Dual Variable Change & $58.33(43)$ & 1.36 & .059 & 0.97 & $0.05(0.00,0.08)$ & .454 & 0.07 \\
\hline PTSD Intrusion Dual Variable Change & $45.47(43)$ & 1.06 & .370 & 1.00 & $0.02(0.00,0.06)$ & .848 & 0.06 \\
\hline PTSD Hyperarousal Dual Variable Change & $55.85(43)$ & 1.30 & .090 & 0.97 & $0.05(0.00,0.08)$ & .536 & 0.08 \\
\hline PTSD Avoidance Dual Variable Change & $47.55(43)$ & 1.11 & .293 & 0.99 & $0.03(0.00,0.07)$ & .797 & 0.06 \\
\hline PTSD Negative Shifts Dual Variable Change & $63.85(43)$ & 1.48 & .021 & 0.96 & $0.06(0.02,0.09)$ & .288 & 0.07 \\
\hline
\end{tabular}


Note. $\mathrm{PTSD}=$ posttraumatic stress disorder; PTSD negative shifts $=$ negative shifts in cognitions and mood; $\chi^{2}=$ Chi Square statistic; CFI = Comparative Fit Index; RMSEA = Root Mean Square Error of Approximation along with 90\% confidence intervals; PCLOSE = $p$ of Close Fit; SRMR $=$ Standardized Root Mean Square Residual. 
Table 19

Bivariate Latent Difference Score Model for PTSD Total Symptom Severity with Alcohol Use Frequency

\begin{tabular}{|c|c|c|c|c|c|c|}
\hline & \multicolumn{3}{|c|}{ PTSD Total Severity } & \multicolumn{3}{|c|}{ Alcohol Use Frequency } \\
\hline & Estimate & $S E$ & $p$ & Estimate & $S E$ & $p$ \\
\hline \multicolumn{7}{|l|}{ Parameter estimates } \\
\hline Initial status means & 56.30 & 3.23 & $<.001$ & -0.51 & 0.05 & $<.001$ \\
\hline Constant change means (nonstationarity) & 13.85 & 8.41 & .100 & -0.12 & 0.13 & .364 \\
\hline Initial status with constant change & 203.20 & 67.50 & .003 & 0.05 & 0.03 & .106 \\
\hline \multicolumn{7}{|l|}{ Proportional (autoregressive) change } \\
\hline$\alpha_{1}$ & -0.25 & 0.11 & .029 & -0.53 & 0.25 & .036 \\
\hline$\alpha_{2}$ & -0.48 & 0.10 & $<.001$ & -0.53 & 0.25 & .036 \\
\hline$\alpha_{3}$ & -0.43 & 0.13 & .001 & -0.53 & 0.25 & .036 \\
\hline$\alpha_{4}$ & -0.60 & 0.13 & $<.001$ & -0.53 & 0.25 & .036 \\
\hline \multicolumn{7}{|l|}{ PTSD - alcohol use associations } \\
\hline Initial status PTSD with initial status alcohol use & \multicolumn{6}{|c|}{ Est. $=-1.76, \quad S E=1.57, \quad p=.260$} \\
\hline Initial status PTSD with constant change alcohol use & \multicolumn{6}{|c|}{ Est. $=1.64$} \\
\hline
\end{tabular}


Constant change PTSD with initial status alcohol use

PTSD - alcohol use cross-lagged effects

\begin{tabular}{|c|c|c|c|c|c|c|}
\hline$\gamma_{1}$ & 18.84 & 18.59 & .311 & $<-0.01$ & $<0.01$ & .135 \\
\hline$\gamma_{2}$ & 10.63 & 17.37 & .541 & $<-0.01$ & $<0.01$ & .013 \\
\hline$\gamma_{3}$ & 10.35 & 17.10 & .545 & $<-0.01$ & $<0.01$ & .039 \\
\hline$\gamma_{4}$ & 10.56 & 17.41 & .544 & $<-0.01$ & $<0.01$ & .188 \\
\hline Error variance & 158.15 & 16.22 & $<.001$ & 0.05 & 0.01 & $<.001$ \\
\hline
\end{tabular}

Note. Model consisted of pairing of the dual variable proportional latent difference score (LDS) PTSD total symptom severity and the dual fixed proportional change LDS alcohol use frequency models. PTSD = posttraumatic stress disorder; $\alpha_{1-4}=$ proportional change parameters; $\gamma_{1-4}=$ cross-lagged effects.

$$
\text { Est. }=-1.22, S E=1.99, p=.540
$$


Table 20

Bivariate Latent Difference Score Model for PTSD Intrusion Cluster Severity with Alcohol Use Frequency

\begin{tabular}{|c|c|c|c|c|c|c|}
\hline & \multicolumn{3}{|c|}{ PTSD Intrusion } & \multicolumn{3}{|c|}{ Alcohol Use Frequency } \\
\hline & Estimate & $S E$ & $p$ & Estimate & $S E$ & $p$ \\
\hline \multicolumn{7}{|l|}{ Parameter estimates } \\
\hline Initial status means & 14.42 & 0.89 & $<.001$ & -0.50 & 0.05 & $<.001$ \\
\hline Constant change means (nonstationarity) & 4.41 & 2.51 & .079 & -0.17 & 0.12 & .145 \\
\hline Initial status with constant change & 12.84 & 6.07 & .034 & 0.05 & 0.03 & .040 \\
\hline \multicolumn{7}{|l|}{ Proportional (autoregressive) change } \\
\hline$\alpha_{1}$ & -0.19 & 0.14 & .183 & -0.58 & 0.22 & .009 \\
\hline$\alpha_{2}$ & -0.31 & 0.13 & .019 & -0.58 & 0.22 & .009 \\
\hline$\alpha_{3}$ & -0.48 & 0.14 & $<.001$ & -0.58 & 0.22 & .009 \\
\hline$\alpha_{4}$ & -0.49 & 0.17 & .004 & -0.58 & 0.22 & .009 \\
\hline \multicolumn{7}{|l|}{ PTSD - alcohol use associations } \\
\hline Initial status PTSD with initial status alcohol use & \multicolumn{6}{|c|}{ Est. $=-0.56, \quad S E=0.43, p=.192$} \\
\hline Initial status PTSD with constant change alcohol use & \multicolumn{3}{|c|}{ Est. $=0.38$} & $S E=0.32, \quad p=$ & 38 & \\
\hline
\end{tabular}


Constant change PTSD with initial status alcohol use

PTSD - alcohol use cross-lagged effects

\begin{tabular}{|c|c|c|c|c|c|c|}
\hline$\gamma_{1}$ & 10.27 & 5.04 & .042 & -0.01 & $<0.01$ & .180 \\
\hline$\gamma_{2}$ & 7.48 & 4.69 & .111 & -0.01 & 0.01 & .033 \\
\hline$\gamma_{3}$ & 5.54 & 4.60 & .229 & -0.01 & 0.01 & .050 \\
\hline$\gamma_{4}$ & 6.80 & 4.88 & .163 & -0.01 & 0.01 & .294 \\
\hline Error variance & 14.77 & 1.56 & $<.001$ & 0.05 & 0.01 & $<.001$ \\
\hline
\end{tabular}

Note. Model consisted of pairing of the dual variable proportional latent difference score (LDS) PTSD intrusion cluster severity and the dual fixed proportional change LDS alcohol use frequency models. PTSD = posttraumatic stress disorder; $\alpha_{1-4}=$ proportional change parameters; $\gamma_{1-4}=$ cross-lagged effects.

$$
\text { Est. }=-0.66, S E=0.53, p=.211
$$


Table 21

Bivariate Latent Difference Score Model for PTSD Hyperarousal Cluster Severity with Alcohol Use Frequency

\begin{tabular}{|c|c|c|c|c|c|c|}
\hline & \multicolumn{3}{|c|}{ PTSD Hyperarousal } & \multicolumn{3}{|c|}{ Alcohol Use Frequency } \\
\hline & Estimate & $S E$ & $p$ & Estimate & $S E$ & $p$ \\
\hline \multicolumn{7}{|l|}{ Parameter estimates } \\
\hline Initial status means & 14.99 & 1.07 & $<.001$ & -0.52 & 0.05 & $<.001$ \\
\hline Constant change means (nonstationarity) & 1.65 & 3.13 & .598 & -0.10 & 0.10 & .311 \\
\hline Initial status with constant change & 15.28 & 7.50 & .042 & 0.04 & 0.02 & .079 \\
\hline \multicolumn{7}{|l|}{ Proportional (autoregressive) change } \\
\hline$\alpha_{1}$ & -0.29 & 0.18 & .098 & -0.51 & 0.20 & .010 \\
\hline$\alpha_{2}$ & -0.44 & 0.14 & .002 & -0.51 & 0.20 & .010 \\
\hline$\alpha_{3}$ & -0.48 & 0.17 & .006 & -0.51 & 0.20 & .010 \\
\hline$\alpha_{4}$ & -0.60 & 0.19 & .002 & -0.51 & 0.20 & .010 \\
\hline \multicolumn{7}{|l|}{ PTSD - alcohol use associations } \\
\hline Initial status PTSD with initial status alcohol use & \multicolumn{6}{|c|}{ Est. $=-0.67$} \\
\hline Initial status PTSD with constant change alcohol use & \multicolumn{6}{|c|}{ Est. $=0.68$} \\
\hline
\end{tabular}


Constant change PTSD with initial status alcohol use

PTSD - alcohol use cross-lagged effects

\begin{tabular}{|c|c|c|c|c|c|c|}
\hline$\gamma_{1}$ & -1.55 & 8.79 & .861 & -0.01 & 0.01 & .118 \\
\hline$\gamma_{2}$ & 0.09 & 7.93 & .991 & -0.01 & 0.01 & .005 \\
\hline$\gamma_{3}$ & -1.21 & 7.31 & .868 & -0.01 & 0.01 & .026 \\
\hline$\gamma_{4}$ & -1.45 & 7.53 & .847 & -0.01 & 0.01 & .091 \\
\hline Error variance & 21.71 & 2.16 & $<.001$ & 0.05 & 0.01 & $<.001$ \\
\hline
\end{tabular}

Note. Model consisted of pairing of the dual variable proportional latent difference score (LDS) PTSD hyperarousal cluster severity and the dual fixed proportional change LDS alcohol use frequency models. PTSD $=$ posttraumatic stress disorder; $\alpha_{1-4}=$ proportional change parameters; $\gamma_{1-4}=$ cross-lagged effects.

Est. $=0.04, S E=0.89, \quad p=.961$ 
Table 22

Bivariate Latent Difference Score Model for PTSD Avoidance Cluster Severity with Alcohol Use Frequency

PTSD Avoidance

Estimate $\quad S E \quad p \quad$ Estimate $\quad S E \quad p$

Parameter estimates

Initial status means

Constant change means (nonstationarity)

Initial status with constant change

Proportional (autoregressive) change
$\alpha_{2}$
$\alpha_{3}$

$\alpha_{1}$

$\alpha_{4}$

PTSD - alcohol use associations

Initial status PTSD with initial status alcohol use

Initial status PTSD with constant change alcohol use

$\begin{array}{llllll}6.62 & 0.50 & <.001 & -0.51 & 0.05 & <.001 \\ 0.66 & 1.50 & .663 & -0.14 & 0.11 & .203 \\ 1.35 & 1.70 & .426 & 0.04 & 0.03 & .127 \\ -0.06 & 0.21 & .780 & -0.45 & 0.22 & .040 \\ -0.33 & 0.19 & .076 & -0.45 & 0.22 & .040 \\ -0.33 & 0.22 & .129 & -0.45 & 0.22 & .040 \\ -0.57 & 0.21 & .008 & -0.45 & 0.22 & .040\end{array}$

$$
\begin{aligned}
& \text { Est. }=-0.35, \quad S E=0.23, p=.126 \\
& \text { Est. }=-0.01, \quad S E=0.16, p=.964
\end{aligned}
$$


Constant change PTSD with initial status alcohol use

PTSD - alcohol use cross-lagged effects

\begin{tabular}{|c|c|c|c|c|c|c|}
\hline$\gamma_{1}$ & 2.81 & 3.13 & .370 & -0.01 & 0.01 & .561 \\
\hline$\gamma_{2}$ & 0.17 & 3.08 & .955 & -0.02 & 0.01 & .132 \\
\hline$\gamma_{3}$ & 0.70 & 3.06 & .818 & -0.02 & 0.02 & .301 \\
\hline$\gamma_{4}$ & 0.68 & 3.03 & .823 & $<0.01$ & 0.02 & .986 \\
\hline Error variance & 7.96 & 0.79 & $<.001$ & 0.05 & 0.01 & $<.001$ \\
\hline
\end{tabular}

Note. Model consisted of pairing of the dual variable proportional latent difference score (LDS) PTSD avoidance cluster severity and the dual fixed proportional change LDS alcohol use frequency models. PTSD = posttraumatic stress disorder; $\alpha_{1-4}=$ proportional change parameters; $\gamma_{1-4}=$ cross-lagged effects.

$$
\text { Est. }=-0.02, S E=0.35, p=.956
$$


Table 23

Bivariate Latent Difference Score Model for PTSD Negative Shifts in Cognitions and Mood Cluster Severity with Alcohol Use Frequency

\begin{tabular}{|c|c|c|c|c|c|c|}
\hline & \multicolumn{3}{|c|}{ PTSD Negative Shifts } & \multicolumn{3}{|c|}{ Alcohol Use Frequency } \\
\hline & Estimate & $S E$ & $p$ & Estimate & $S E$ & $p$ \\
\hline \multicolumn{7}{|l|}{ Parameter estimates } \\
\hline Initial status means & 17.07 & 1.35 & $<.001$ & -0.51 & 0.05 & $<.001$ \\
\hline Constant change means (nonstationarity) & 2.92 & 4.16 & .483 & -0.06 & 0.13 & .662 \\
\hline Initial status with constant change & 33.37 & 12.75 & .009 & 0.03 & 0.03 & .273 \\
\hline \multicolumn{7}{|l|}{ Proportional (autoregressive) change } \\
\hline$\alpha_{1}$ & -0.29 & 0.14 & .045 & -0.37 & 0.22 & .089 \\
\hline$\alpha_{2}$ & -0.64 & 0.14 & $<.001$ & -0.37 & 0.22 & .089 \\
\hline$\alpha_{3}$ & -0.44 & 0.20 & .030 & -0.37 & 0.22 & .089 \\
\hline$\alpha_{4}$ & -0.66 & 0.18 & $<.001$ & -0.37 & 0.22 & .089 \\
\hline \multicolumn{7}{|l|}{ PTSD - alcohol use associations } \\
\hline
\end{tabular}


Initial status PTSD with constant change alcohol use

Constant change PTSD with initial status alcohol use

PTSD - alcohol use cross-lagged effects$$
\gamma_{1}
$$$$
\gamma_{2}
$$$$
\gamma_{3}
$$$$
\gamma_{4}
$$

Error variance

$$
\begin{aligned}
& \text { Est. }=0.82, \quad S E=0.62, p=.187 \\
& \text { Est. }=-0.21, \quad S E=1.03, p=.842
\end{aligned}
$$

$\begin{array}{rrrrrr}2.22 & 8.36 & .791 & -0.01 & 0.01 & .288 \\ -1.15 & 8.37 & .881 & -0.01 & 0.01 & .040 \\ 0.24 & 8.45 & .977 & -0.01 & 0.01 & .108 \\ -0.07 & 8.38 & .993 & -0.01 & 0.01 & .230 \\ 37.03 & 3.70 & <.001 & 0.05 & 0.01 & <.001\end{array}$

Note. Model consisted of pairing of the dual variable proportional latent difference score (LDS) PTSD negative shifts in cognitions and mood cluster severity and the dual fixed proportional change LDS alcohol use frequency models. PTSD = posttraumatic stress disorder; PTSD negative shifts $=$ PTSD negative shifts in cognitions and mood; $\alpha_{1-4}=$ proportional change parameters; $\gamma_{1-4}=$ crosslagged effects. 
participants with higher scores for PTSD negative shifts in cognitions and mood at the second time interval exhibited greater decreases (or smaller increases) in alcohol consumption over the subsequent time interval. When change in PTSD was regressed on alcohol use, there was one significant association: Alcohol use in the first 4 months posttrauma was a positive predictor of change in PTSD intrusion symptoms between 4 and 8 months posttrauma. This suggests that participants with higher alcohol consumption in the first four months posttrauma had a greater increase (or smaller decrease) in PTSD intrusion symptoms over the subsequent 4 months.

Figure 7 depicts the path diagram for the PTSD with drug use frequency BLDS models in which the dual variable proportional change LDS model was retained for the PTSD components (i.e., total and cluster scores) and the fixed proportional change LDS model was retained for drug use frequency. Parameter estimates for the PTSD with drug use BLDS models are contained in Tables 24 through 28, while model fit statistics are found in Table 18. All models demonstrated a good model-to-data fit.

The BLDS models for PTSD components with drug use were fairly consistent. In all models, the initial status means were significantly different from 0 . Also, with the exception of the PTSD avoidance BLDS model, the constant change components for PTSD were significant and positive, suggesting a general trend of increased PTSD over time when controlling for the presence of prior PTSD symptoms and the relevant features of drug use. All proportional change parameters for PTSD components (i.e., total and cluster scores) were significant and negative, indicating that higher PTSD severity was associated with greater subsequent decreases (or smaller increases) in PTSD severity. The BLDS model involving PTSD avoidance demonstrated significant and negative drug use proportional change across time; the proportional change component for all other drug use models were negligible. With respect to cross-lagged effects, 


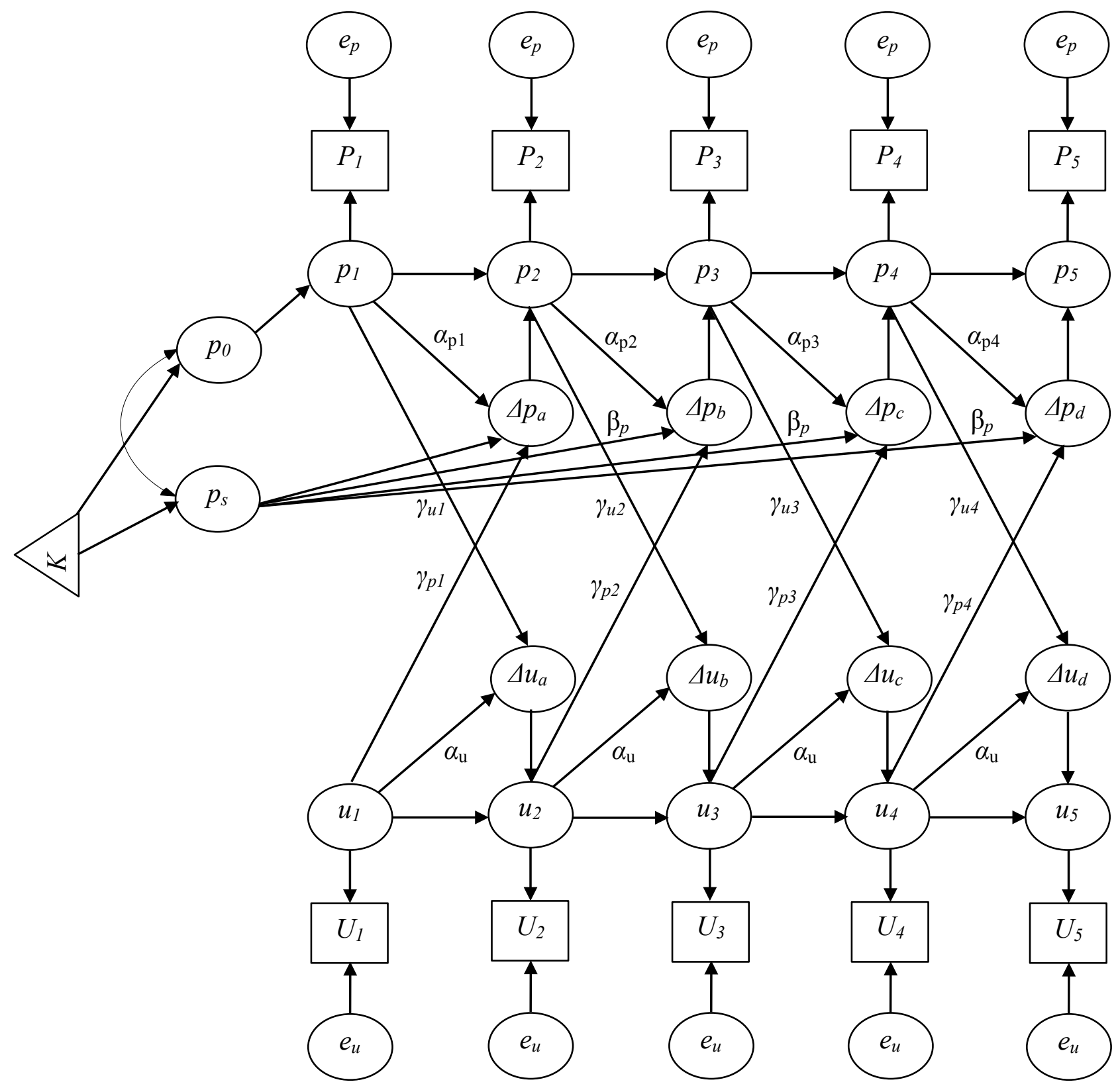

Figure 7. Bivariate latent difference score model examining associations between PTSD and drug use subsequent to traumatic exposure. This BLDS model was the result of pairing the best fitting univariate LDS models for PTSD components (i.e., total and PTSD clusters) and drug use frequency: The dual variable proportional change LDS model was retained for PTSD and the fixed proportional change LDS model was retained for drug use. $K$ represents a constant used to 
estimate means and intercepts. $P_{[1-5]}$ denotes the observed PTSD scores at each time point, $p_{[1-5]}$ represents the true score latent variables for each time point, $\Delta p_{[a-d]}$ represents the latent difference scores between corresponding time points, $p_{0}$ denotes the estimated initial status mean for PTSD, $\beta_{p}$ represents the effect of PTSD nonstationarity (i.e., constant change) on PTSD latent difference scores, $\alpha_{p[1-4]}$ represents the proportional change as a functioning of the prior true score latent variable PTSD status on the subsequent latent difference score, and $e_{\mathrm{p}}$ represents the error terms for PTSD, which were constrained to be equal across time points. $U_{[1-5]}$ denotes the observed substance use scores at each time point, $u_{[1-5]}$ represents the true score latent variables for each time point, $\Delta u_{[a-d]}$ represents the latent difference scores between corresponding time points, $\alpha_{u}$ represents the (fixed) proportional change as a functioning of the prior true score latent variable substance use status on the subsequent latent difference score, and $e_{\mathrm{u}}$ represents the error terms for substance use, which were constrained to be equal across time points. $\gamma_{p[1-4]}$ denotes the cross-lagged influence of true PTSD scores on substance use latent difference scores across time points, while $\gamma_{u[1-4]}$ denotes the cross-lagged influence of true substance use scores on PTSD latent difference scores across time points. 
Table 24

Bivariate Latent Difference Score Model for PTSD Total Symptom Severity with Drug Use Frequency

\begin{tabular}{|c|c|c|c|c|c|c|}
\hline & \multicolumn{3}{|c|}{ PTSD Total Severity } & \multicolumn{3}{|c|}{ Drug Use Frequency } \\
\hline & Estimate & $S E$ & $p$ & Estimate & $S E$ & $p$ \\
\hline \multicolumn{7}{|l|}{ Parameter estimates } \\
\hline Initial status means & 56.40 & 3.32 & $<.001$ & -0.20 & 0.04 & $<.001$ \\
\hline Constant change means (nonstationarity) & 12.15 & 4.07 & .003 & -- & -- & -- \\
\hline Initial status with constant change & 247.27 & 67.30 & $<.001$ & -- & -- & -- \\
\hline \multicolumn{7}{|l|}{ Proportional (autoregressive) change } \\
\hline$\alpha_{1}$ & -0.37 & 0.08 & $<.001$ & -0.05 & 0.03 & .099 \\
\hline$\alpha_{2}$ & -0.54 & 0.09 & $<.001$ & -0.05 & 0.03 & .099 \\
\hline$\alpha_{3}$ & -0.51 & 0.11 & $<.001$ & -0.05 & 0.03 & .099 \\
\hline$\alpha_{4}$ & -0.67 & 0.12 & $<.001$ & -0.05 & 0.03 & .099 \\
\hline \multicolumn{7}{|l|}{ PTSD - drug use cross-lagged effects } \\
\hline$\gamma_{1}$ & 5.61 & 7.71 & .467 & $<-0.01$ & $<0.01$ & .278 \\
\hline$\gamma_{2}$ & 1.34 & 6.75 & .843 & $<0.01$ & $<0.01$ & .166 \\
\hline
\end{tabular}




$\begin{array}{lrrrrrr}\gamma_{3} & 5.69 & 7.15 & .426 & <-0.01 & <0.01 & .010 \\ \gamma_{4} & 8.63 & 9.36 & .357 & <0.01 & <0.01 & .718 \\ \text { Error variance } & 161.29 & 16.05 & <.001 & 0.04 & <0.01 & <.001\end{array}$

Note. Model consisted of pairing of the dual variable proportional latent difference score (LDS) PTSD total symptom severity and the fixed proportional change LDS drug use frequency models. “--" denotes parameters that were not estimate due to the absence of the constant change component for drug use. PTSD = posttraumatic stress disorder; $\alpha_{1-4}=$ proportional change parameters; $\gamma_{1-4}=$ crosslagged effects. 
Table 25

Bivariate Latent Difference Score Model for PTSD Intrusion Cluster Severity with Drug Use Frequency

\begin{tabular}{|c|c|c|c|c|c|c|}
\hline & \multicolumn{3}{|c|}{ PTSD Intrusion } & \multicolumn{3}{|c|}{ Drug Use Frequency } \\
\hline & Estimate & $S E$ & $p$ & Estimate & $S E$ & $p$ \\
\hline \multicolumn{7}{|l|}{ Parameter estimates } \\
\hline Initial status means & 14.41 & 0.91 & $<.001$ & -0.20 & 0.04 & $<.001$ \\
\hline Constant change means (nonstationarity) & 2.61 & 1.20 & .029 & -- & -- & -- \\
\hline Initial status with constant change & 17.20 & 5.58 & .002 & -- & -- & -- \\
\hline \multicolumn{7}{|l|}{ Proportional (autoregressive) change } \\
\hline$\alpha_{1}$ & -0.38 & 0.09 & $<.001$ & -0.40 & 0.03 & .133 \\
\hline$\alpha_{2}$ & -0.41 & 0.11 & $<.001$ & -0.40 & 0.03 & .133 \\
\hline$\alpha_{3}$ & -0.57 & 0.12 & $<.001$ & -0.40 & 0.03 & .133 \\
\hline$\alpha_{4}$ & -0.64 & 0.15 & $<.001$ & -0.40 & 0.03 & .133 \\
\hline \multicolumn{7}{|l|}{ PTSD - drug use cross-lagged effects } \\
\hline$\gamma_{1}$ & 2.98 & 2.12 & .160 & $<-0.01$ & $<0.01$ & .198 \\
\hline$\gamma_{2}$ & 2.00 & 1.97 & .311 & $<0.01$ & $<0.01$ & .128 \\
\hline
\end{tabular}




$\begin{array}{lrrrrrr}\gamma_{3} & 1.86 & 1.98 & .349 & -0.01 & <0.01 & .021 \\ \gamma_{4} & 3.30 & 2.77 & .233 & <0.01 & <0.01 & .810 \\ \text { Error variance } & 15.62 & 1.55 & <.001 & 0.04 & <0.01 & <.001\end{array}$

Note. Model consisted of pairing of the dual variable proportional latent difference score (LDS) PTSD intrusion cluster severity and the fixed proportional change LDS drug use frequency models. "--" denotes parameters that were not estimate due to the absence of the constant change component for drug use. PTSD $=$ posttraumatic stress disorder; $\alpha_{1-4}=$ proportional change parameters; $\gamma_{1-4}=$ crosslagged effects. 
Table 26

Bivariate Latent Difference Score Model for PTSD Hyperarousal Cluster Severity with Drug Use Frequency

\begin{tabular}{|c|c|c|c|c|c|c|}
\hline & \multicolumn{3}{|c|}{ PTSD Hyperarousal } & \multicolumn{3}{|c|}{ Drug Use Frequency } \\
\hline & Estimate & $S E$ & $p$ & Estimate & $S E$ & $p$ \\
\hline \multicolumn{7}{|l|}{ Parameter estimates } \\
\hline Initial status means & 14.98 & 1.07 & $<.001$ & -0.19 & 0.04 & $<.001$ \\
\hline Constant change means (nonstationarity) & 2.84 & 1.37 & .038 & -- & -- & -- \\
\hline Initial status with constant change & 18.07 & 6.77 & .008 & -- & -- & -- \\
\hline \multicolumn{7}{|l|}{ Proportional (autoregressive) change } \\
\hline$\alpha_{1}$ & -0.29 & 0.10 & .005 & -0.05 & 0.03 & .101 \\
\hline$\alpha_{2}$ & -0.51 & 0.11 & $<.001$ & -0.05 & 0.03 & .101 \\
\hline$\alpha_{3}$ & -0.52 & 0.14 & $<.001$ & -0.05 & 0.03 & .101 \\
\hline$\alpha_{4}$ & -0.59 & 0.15 & $<.001$ & -0.05 & 0.03 & .101 \\
\hline \multicolumn{7}{|l|}{ PTSD - drug use cross-lagged effects } \\
\hline$\gamma_{1}$ & 2.19 & 2.67 & .411 & $<-0.01$ & $<0.01$ & .179 \\
\hline$\gamma_{2}$ & 1.32 & 2.28 & .564 & $<0.01$ & $<0.01$ & .225 \\
\hline
\end{tabular}




\begin{tabular}{|c|c|c|c|c|c|c|}
\hline$\gamma_{3}$ & 0.97 & 2.36 & .681 & -0.01 & $<0.01$ & .029 \\
\hline$\gamma_{4}$ & 4.26 & 3.38 & .207 & $<0.01$ & $<0.01$ & .962 \\
\hline Error variance & 21.74 & 2.20 & $<.001$ & 0.04 & $<0.01$ & $<.001$ \\
\hline
\end{tabular}


Table 27

Bivariate Latent Difference Score Model for PTSD Avoidance Cluster Severity with Drug Use Frequency

\begin{tabular}{|c|c|c|c|c|c|c|}
\hline & \multicolumn{3}{|c|}{ PTSD Avoidance } & \multicolumn{3}{|c|}{ Drug Use Frequency } \\
\hline & Estimate & $S E$ & $p$ & Estimate & $S E$ & $p$ \\
\hline \multicolumn{7}{|l|}{ Parameter estimates } \\
\hline Initial status means & 6.61 & 0.53 & $<.001$ & -0.20 & 0.04 & $<.001$ \\
\hline Constant change means (nonstationarity) & 0.70 & 0.72 & .333 & -- & -- & -- \\
\hline Initial status with constant change & 2.20 & 1.60 & .168 & -- & -- & -- \\
\hline \multicolumn{7}{|l|}{ Proportional (autoregressive) change } \\
\hline$\alpha_{1}$ & -0.26 & 0.13 & .040 & -0.06 & 0.03 & .038 \\
\hline$\alpha_{2}$ & -0.44 & 0.15 & .003 & -0.06 & 0.03 & .038 \\
\hline$\alpha_{3}$ & -0.36 & 0.18 & .047 & -0.06 & 0.03 & .038 \\
\hline$\alpha_{4}$ & -0.69 & 0.17 & $<.001$ & -0.06 & 0.03 & .038 \\
\hline \multicolumn{7}{|l|}{ PTSD - drug use cross-lagged effects } \\
\hline$\gamma_{1}$ & 0.38 & 1.29 & .770 & -0.01 & 0.01 & .237 \\
\hline$\gamma_{2}$ & -2.50 & 1.29 & .052 & 0.01 & $<0.01$ & .213 \\
\hline
\end{tabular}




$\begin{array}{lrrrrrr}\gamma_{3} & 2.32 & 1.55 & .135 & -0.02 & 0.01 & .007 \\ \gamma_{4} & -0.28 & 1.82 & .879 & <0.01 & 0.01 & .574 \\ \text { Error variance } & 7.81 & 0.76 & <.001 & 0.04 & <0.01 & <.001\end{array}$

Note. Model consisted of pairing of the dual variable proportional latent difference score (LDS) PTSD avoidance cluster severity and the fixed proportional change LDS drug use frequency models. "--" denotes parameters that were not estimate due to the absence of the constant change component for drug use. PTSD $=$ posttraumatic stress disorder; $\alpha_{1-4}=$ proportional change parameters; $\gamma_{1-4}=$ crosslagged effects. 
Table 28

Bivariate Latent Difference Score Model for PTSD Negative Shifts in Cognitions and Mood Cluster Severity with Drug Use Frequency

\begin{tabular}{|c|c|c|c|c|c|c|}
\hline & \multicolumn{3}{|c|}{ PTSD Negative Shifts } & \multicolumn{3}{|c|}{ Drug Use Frequency } \\
\hline & Estimate & $S E$ & $p$ & Estimate & $S E$ & $p$ \\
\hline \multicolumn{7}{|l|}{ Parameter estimates } \\
\hline Initial status means & 16.98 & 1.40 & $<.001$ & -0.22 & 0.04 & $<.001$ \\
\hline Constant change means (nonstationarity) & 3.90 & 1.61 & .016 & -- & -- & -- \\
\hline Initial status with constant change & 40.39 & 12.78 & .002 & -- & -- & -- \\
\hline \multicolumn{7}{|l|}{ Proportional (autoregressive) change } \\
\hline$\alpha_{1}$ & -0.41 & 0.11 & $<.001$ & -0.04 & 0.03 & .151 \\
\hline$\alpha_{2}$ & -0.69 & 0.13 & $<.001$ & -0.04 & 0.03 & .151 \\
\hline$\alpha_{3}$ & -0.54 & 0.17 & .002 & -0.04 & 0.03 & .151 \\
\hline$\alpha_{4}$ & -0.74 & 0.16 & $<.001$ & -0.04 & 0.03 & .151 \\
\hline \multicolumn{7}{|l|}{ PTSD - drug use cross-lagged effects } \\
\hline$\gamma_{1}$ & -0.90 & 3.37 & .789 & $<-0.01$ & $<0.01$ & .587 \\
\hline$\gamma_{2}$ & -0.41 & 2.97 & .891 & $<0.01$ & $<0.01$ & .153 \\
\hline
\end{tabular}




\begin{tabular}{|c|c|c|c|c|c|c|}
\hline$\gamma_{3}$ & 0.61 & 3.14 & .846 & -0.01 & $<0.01$ & .004 \\
\hline$\gamma_{4}$ & 1.67 & 4.07 & .681 & $<0.01$ & $<0.01$ & .558 \\
\hline Error variance & 36.95 & 3.62 & $<.001$ & 0.04 & $<0.01$ & $<.001$ \\
\hline
\end{tabular}


none of the drug use to change in PTSD coefficients were significant, which suggested a lack of unique association between prior drug use and subsequent change in PTSD. However, the third PTSD to change in drug use cross-lagged effect was significant and negative in all drug use models. As such, participants with higher PTSD scores between 8 and 12 months were expected to have greater decreases (or smaller increases) in the frequency of drug use over the 12 to 16 months time interval.

Self-medication versus susceptibility theory models: Hypothesis 3c. When PTSD with alcohol use BLDS models reflecting the self-medication theory (i.e., cross lagged effects from alcohol use to change in PTSD were constrained to 0) and the susceptibility theory (i.e., cross lagged effects from PTSD to change in alcohol use were constrained to 0) were compared, models representing the susceptibility theory resulted in a statistically poorer fit to the data as compared to models representing the self-medication theory for the following PTSD components: PTSD total severity, hyperarousal, avoidance, and negative shifts in cognitions and mood. While both the self-medication theory and susceptibility theory models resulted in a good fit to the data for PTSD intrusion with alcohol use, the deviance statistic was smaller for the susceptibility model and, thus, a better fit to the data (Table 29).

Parameter estimates for the self-medication theory PTSD with alcohol use BLDS models (i.e., for PTSD total, hyperarousal, avoidance, and negative shifts in cognitions and mood PTSD components) are contained in Tables 30 through 33. Significance of parameter estimates were comparable to the abovementioned PTSD with alcohol use BLDS models contained in Tables 19 and 21 through 23. With respect to cross-lagged coupling effects, the second and third PTSD to change in alcohol use cross-lagged effects were significant and negative in the PTSD hyperarousal with alcohol use model. As such, participants with higher PTSD scores at the 
Table 29

Goodness of Fit Estimates for Self-Medication Theory and Susceptibility Theory Bivariate Latent Difference Score Models Alcohol Use Dual Fixed Change with PTSD (component) Dual Variable Proportional Change

\begin{tabular}{|c|c|c|c|c|c|c|c|c|}
\hline PTSD Model: & $\chi^{2}(d f)$ & $\chi^{2} / d f$ & $p$ & CFI & RMSEA (90\% CI) & PCLOSE & SRMR & AIC \\
\hline Total Severity (SMed) & $55.13(40)$ & 1.38 & .056 & 0.97 & $0.05(0.00,0.08)$ & .424 & 0.06 & 4392.48 \\
\hline Total Severity (Susc) & $61.89(40)$ & 1.55 & .010 & 0.96 & $0.06(0.03,0.09)$ & .229 & 0.07 & 4399.24 \\
\hline Intrusion (SMed) & $51.17(40)$ & 1.28 & .111 & 0.98 & $0.05(0.00,0.08)$ & .560 & 0.06 & 3259.17 \\
\hline Intrusion (Susc) & $50.69(40)$ & 1.27 & .120 & 0.98 & $0.04(0.00,0.08)$ & .577 & 0.06 & 3258.70 \\
\hline Hyperarousal (SMed) & $56.57(40)$ & 1.41 & .043 & 0.97 & $0.06(0.01,0.09)$ & .377 & 0.07 & 3398.58 \\
\hline Hyperarousal (Susc) & $66.05(40)$ & 1.65 & .006 & 0.95 & $0.07(0.04,0.10)$ & .144 & 0.09 & 3408.06 \\
\hline Avoidance (SMed) & $41.85(40)$ & 1.05 & .390 & 1.00 & $0.02(0.00,0.06)$ & .849 & 0.07 & 2836.17 \\
\hline Avoidance (Susc) & $46.97(40)$ & 1.17 & .209 & 0.98 & $0.04(0.00,0.07)$ & .704 & 0.06 & 2841.28 \\
\hline Negative Shifts (SMed) & $63.10(40)$ & 1.58 & .011 & 0.95 & $0.07(0.03,0.09)$ & .202 & 0.07 & 3626.57 \\
\hline Negative Shifts (Susc) & $68.93(40)$ & 1.72 & .003 & 0.94 & $0.07(0.04,0.10)$ & .101 & 0.08 & 3632.40 \\
\hline
\end{tabular}




\begin{tabular}{|c|c|c|c|c|c|c|c|c|}
\hline \multicolumn{9}{|c|}{ Drug Use Fixed Proportional Change with PTSD (component) Dual Variable Proportional Change } \\
\hline PTSD Model: & $\chi^{2}(d f)$ & $\chi^{2} / d f$ & $p$ & CFI & RMSEA $(90 \% \mathrm{CI})$ & PCLOSE & SRMR & AIC \\
\hline Total Severity (SMed) & $59.65(47)$ & 1.27 & .102 & 0.98 & $0.04(0.00,0.08)$ & .585 & 0.07 & 4253.89 \\
\hline Total Severity (Susc) & $66.63(47)$ & 1.42 & .031 & 0.97 & $0.06(0.02,0.08)$ & .367 & 0.10 & 4260.86 \\
\hline Intrusion (SMed) & $48.59(47)$ & 1.03 & .409 & 1.00 & $0.02(0.00,0.06)$ & .882 & 0.08 & 3120.38 \\
\hline Intrusion (Susc) & $53.01(47)$ & 1.13 & .254 & 0.99 & $0.03(0.00,0.07)$ & .783 & 0.08 & 3124.80 \\
\hline Hyperarousal (SMed) & $57.84(47)$ & 1.23 & .134 & 0.98 & $0.04(0.00,0.07)$ & .642 & 0.08 & 3265.40 \\
\hline Hyperarousal (Susc) & $63.27(47)$ & 1.35 & .057 & 0.97 & $0.05(0.00,0.08)$ & .469 & 0.10 & 3270.84 \\
\hline Avoidance (SMed) & $52.75(47)$ & 1.12 & .262 & 0.98 & $0.03(0.00,0.07)$ & .790 & 0.08 & 2697.51 \\
\hline Avoidance (Susc) & $56.27(47)$ & 1.20 & .167 & 0.98 & $0.04(0.00,0.07)$ & .691 & 0.09 & 2701.03 \\
\hline Negative Shifts (SMed) & $64.29(47)$ & 1.37 & 0.05 & 0.96 & $0.05(0.01,0.08)$ & .437 & 0.07 & 3485.24 \\
\hline Negative Shifts (Susc) & $72.89(47)$ & 1.55 & .009 & 0.95 & $0.06(0.03,0.09)$ & .210 & 0.08 & 3493.83 \\
\hline
\end{tabular}

Note. $\mathrm{PTSD}=$ posttraumatic stress disorder; SMed = Model reflecting self-medication theory (i.e., cross-lagged coupling effects from PTSD to substance use were estimated while constraining to 0 the cross-lagged coupling effects from substance use to PTSD); Susc $=$ Model reflecting susceptibility theory (i.e., cross-lagged coupling effects from substance use to PTSD were estimated while 
constraining to 0 the cross-lagged coupling effects from PTSD to substance use); Negative Shifts = negative shifts in cognitions and mood PTSD symptom cluster; $\chi^{2}=$ Chi Square statistic; CFI = Comparative Fit Index; RMSEA = Root Mean Square Error of Approximation along with 90\% confidence intervals; PCLOSE $=p$ of Close Fit; SRMR = Standardized Root Mean Square Residual; $\mathrm{AIC}=$ Akaike Information Criterion. 
Table 30

Self-Medication Theory Bivariate Latent Difference Score Model for PTSD Total Symptom Severity with Alcohol Use Frequency

\begin{tabular}{|c|c|c|c|c|c|c|}
\hline & \multicolumn{3}{|c|}{ PTSD Total Severity } & \multicolumn{3}{|c|}{ Alcohol Use Frequency } \\
\hline & Estimate & $S E$ & $p$ & Estimate & $S E$ & $p$ \\
\hline \multicolumn{7}{|l|}{ Parameter estimates } \\
\hline Initial status means & 56.39 & 3.03 & $<.001$ & -0.52 & 0.05 & $<.001$ \\
\hline Constant change means (nonstationarity) & 10.34 & 3.72 & .005 & -0.09 & 0.12 & .425 \\
\hline Initial status with constant change & 230.71 & 62.63 & $<.001$ & 0.04 & 0.02 & .122 \\
\hline \multicolumn{7}{|l|}{ Proportional (autoregressive) change } \\
\hline$\alpha_{1}$ & -0.34 & 0.07 & $<.001$ & -0.45 & 0.21 & .035 \\
\hline$\alpha_{2}$ & -0.51 & 0.08 & $<.001$ & -0.45 & 0.21 & .035 \\
\hline$\alpha_{3}$ & -0.49 & 0.11 & $<.001$ & -0.45 & 0.21 & .035 \\
\hline$\alpha_{4}$ & -0.67 & 0.11 & $<.001$ & -0.45 & 0.21 & .035 \\
\hline \multicolumn{7}{|l|}{ PTSD - alcohol use associations } \\
\hline Initial status PTSD with initial status alcohol use & \multicolumn{6}{|c|}{ Est. $=-1.15$} \\
\hline Initial status PTSD with constant change alcohol use & \multicolumn{6}{|c|}{ Est. $=1.70, S$} \\
\hline
\end{tabular}


Constant change PTSD with initial status alcohol use

PTSD - alcohol use cross-lagged effects

\begin{tabular}{|c|c|c|c|c|c|c|}
\hline$\gamma_{1}$ & -- & -- & -- & $<-0.01$ & $<0.01$ & .243 \\
\hline$\gamma_{2}$ & -- & -- & -- & $<-0.01$ & $<0.01$ & .025 \\
\hline$\gamma_{3}$ & -- & -- & -- & $<-0.01$ & $<0.01$ & .074 \\
\hline$\gamma_{4}$ & -- & -- & -- & $<-0.01$ & $<0.01$ & .283 \\
\hline Error variance & 161.72 & 16.04 & $<.001$ & 0.05 & 0.01 & $<.001$ \\
\hline
\end{tabular}

Note. Model consisted of pairing of the dual variable proportional latent difference score (LDS) PTSD total symptom severity and the dual fixed proportional change LDS alcohol use frequency models, with cross lagged paths from alcohol use to change in PTSD constrained to 0 to reflect the self-medication theory. "--" denotes parameters that were not estimated. PTSD = posttraumatic stress disorder; $\alpha_{1-4}=$ proportional change parameters; $\gamma_{1-4}=$ cross-lagged effects. 
Table 31

Self-Medication Theory Bivariate Latent Difference Score Model for PTSD Hyperarousal Cluster Severity with Alcohol Use Frequency

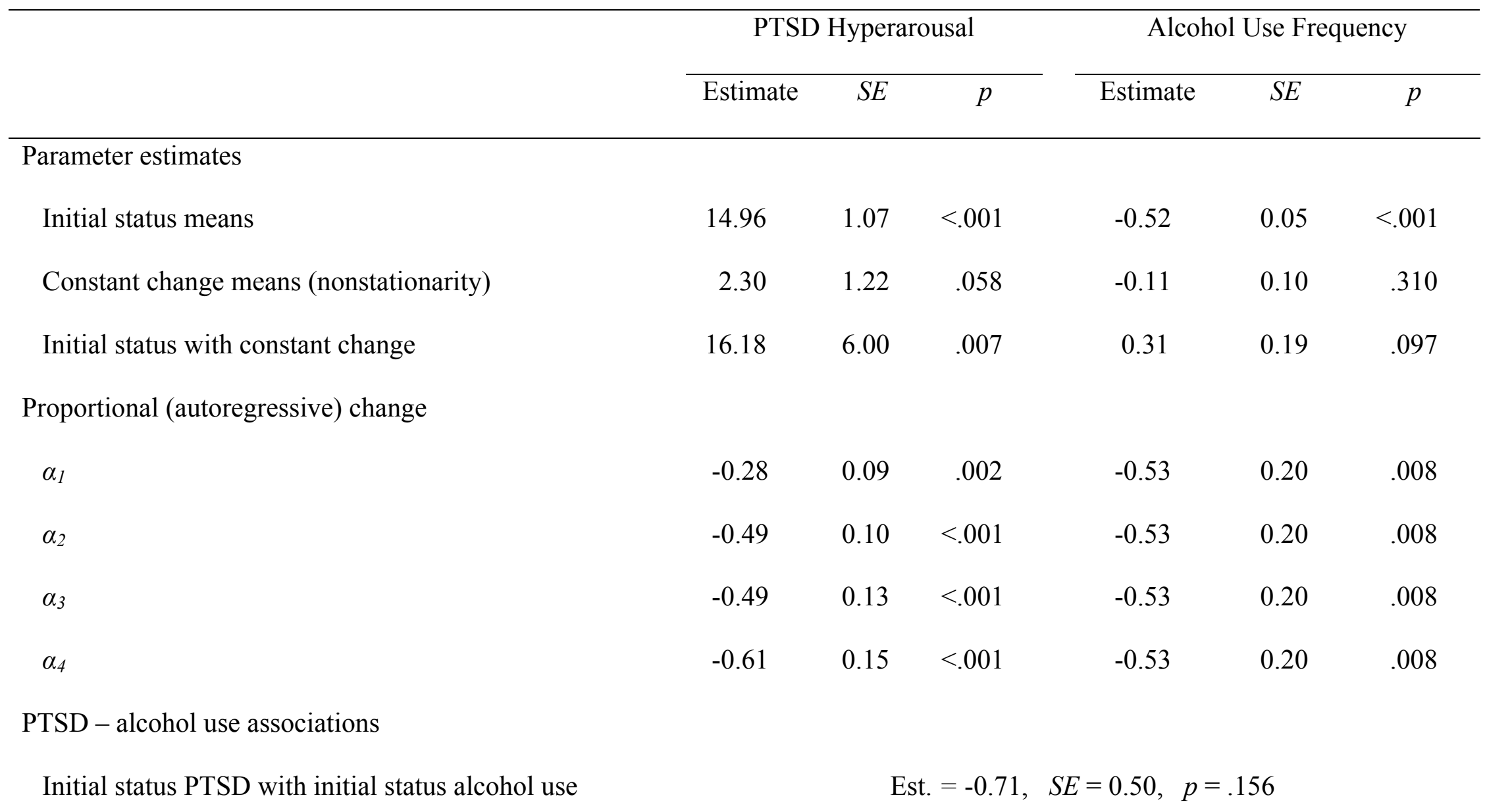


Initial status PTSD with constant change alcohol use

Constant change PTSD with initial status alcohol use

PTSD - alcohol use cross-lagged effects

$\gamma$

$\gamma_{2}$

$\gamma_{3}$

$\gamma_{4}$

Error variance

$$
\begin{aligned}
& \text { Est. }=0.68, S E=0.36, p=.057 \\
& \text { Est. }=0.07, \quad S E=0.25, p=.785
\end{aligned}
$$

$\begin{array}{lll}-- & -- & - \\ -- & -- & - \\ -- & -- & - \\ -- & -- & --\end{array}$

$-0.01$

$<0.01$ .076

21.78

$2.17<.001$

$-0.01$

0.05
$0.01 \quad .003$

$0.01 \quad .018$

$0.01 \quad .071$

Note. Model consisted of pairing of the dual variable proportional latent difference score (LDS) PTSD hyperarousal cluster severity and the dual fixed proportional change LDS alcohol use frequency models, with cross lagged paths from alcohol use to change in PTSD constrained to 0 to reflect the self-medication theory. “--" denotes parameters that were not estimated. PTSD = posttraumatic stress disorder; $\alpha_{1-4}=$ proportional change parameters; $\gamma_{1-4}=$ cross-lagged effects. 
Table 32

Self-Medication Theory Bivariate Latent Difference Score Model for PTSD Avoidance Cluster Severity with Alcohol Use Frequency

\begin{tabular}{|c|c|c|c|c|c|c|}
\hline & \multicolumn{3}{|c|}{ PTSD Avoidance } & \multicolumn{3}{|c|}{ Alcohol Use Frequency } \\
\hline & Estimate & $S E$ & $p$ & Estimate & $S E$ & $p$ \\
\hline \multicolumn{7}{|l|}{ Parameter estimates } \\
\hline Initial status means & 6.59 & 0.52 & $<.001$ & -0.52 & 0.05 & $<.001$ \\
\hline Constant change means (nonstationarity) & 0.58 & 0.72 & .422 & -0.15 & 0.11 & .185 \\
\hline Initial status with constant change & 1.97 & 1.54 & .200 & 0.04 & 0.03 & .148 \\
\hline \multicolumn{7}{|l|}{ Proportional (autoregressive) change } \\
\hline$\alpha_{1}$ & -0.25 & 0.12 & .043 & -0.43 & 0.22 & .052 \\
\hline$\alpha_{2}$ & -0.34 & 0.15 & .021 & -0.43 & 0.22 & .052 \\
\hline$\alpha_{3}$ & -0.39 & 0.17 & .021 & -0.43 & 0.22 & .052 \\
\hline$\alpha_{4}$ & -0.64 & 0.19 & .001 & -0.43 & 0.22 & .052 \\
\hline \multicolumn{7}{|l|}{ PTSD - alcohol use associations } \\
\hline Initial status PTSD with initial status alcohol use & \multicolumn{6}{|c|}{ Est. $=-0.22$} \\
\hline Initial status PTSD with constant change alcohol use & \multicolumn{6}{|c|}{ Est. $=0.01, S E=0.16, p=$} \\
\hline
\end{tabular}


Constant change PTSD with initial status alcohol use

PTSD - alcohol use cross-lagged effects

\begin{tabular}{|c|c|c|c|c|c|c|}
\hline$\gamma_{1}$ & -- & -- & -- & $<-0.01$ & 0.01 & .761 \\
\hline$\gamma_{2}$ & -- & -- & -- & -0.02 & 0.01 & .194 \\
\hline$\gamma_{3}$ & -- & -- & -- & -0.01 & 0.02 & .417 \\
\hline$\gamma_{4}$ & -- & -- & -- & $<0.01$ & 0.02 & .832 \\
\hline Error variance & 8.10 & 0.79 & $<.001$ & 0.05 & 0.01 & $<.001$ \\
\hline
\end{tabular}

Note. Model consisted of pairing of the dual variable proportional latent difference score (LDS) PTSD avoidance cluster severity and the dual fixed proportional change LDS alcohol use frequency models, with cross lagged paths from alcohol use to change in PTSD constrained to 0 to reflect the self-medication theory. "--" denotes parameters that were not estimated. PTSD = posttraumatic stress disorder; $\alpha_{1-4}=$ proportional change parameters; $\gamma_{1-4}=$ cross-lagged effects. 
Table 33

Self-Medication Theory Bivariate Latent Difference Score Model for PTSD Negative Shifts in Cognitions and Mood Cluster Severity with Alcohol Use Frequency

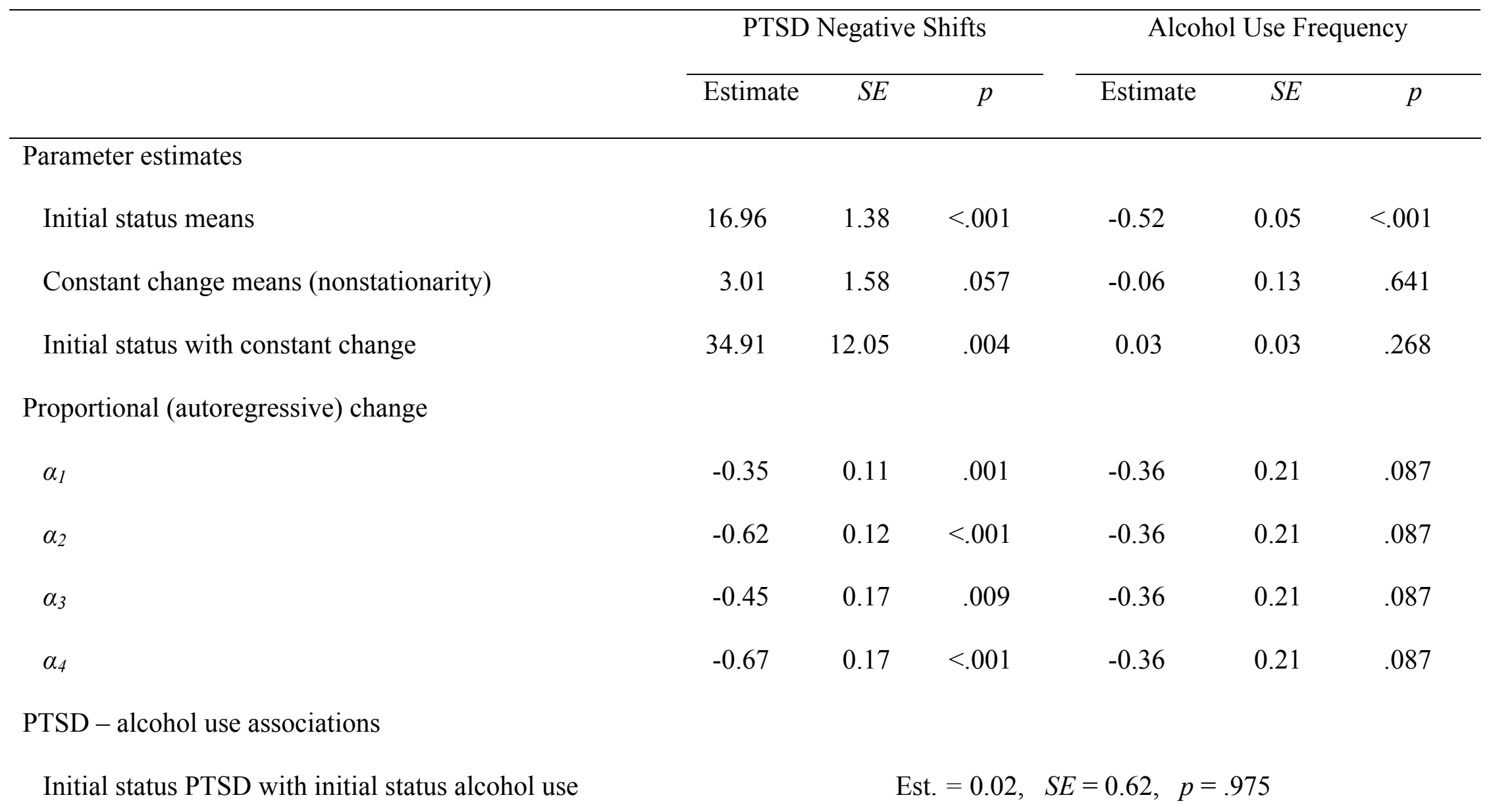


Initial status PTSD with constant change alcohol use

Constant change PTSD with initial status alcohol use

PTSD - alcohol use cross-lagged effects

$\gamma$

$\gamma_{2}$

$\gamma_{3}$

$\gamma_{4}$

Error variance

$$
\begin{aligned}
& \text { Est. }=0.84, S E=0.57, p=.143 \\
& \text { Est. }=-0.18, \quad S E=0.32, p=.562
\end{aligned}
$$

$\begin{array}{llllll}-- & -- & -- & <-0.01 & <0.01 & .313 \\ -- & -- & -- & -0.01 & 0.01 & .038 \\ -- & -- & -- & -0.01 & 0.01 & .107 \\ -- & -- & -- & -0.01 & 0.01 & .246 \\ 37.33 & 3.71 & <.001 & 0.05 & 0.01 & <.001\end{array}$

Note. Model consisted of pairing of the dual variable proportional latent difference score (LDS) PTSD negative shifts in cognitions and mood cluster severity and the dual fixed proportional change LDS alcohol use frequency models, with cross lagged paths from alcohol use to change in PTSD constrained to 0 to reflect the self-medication theory. "--" denotes parameters that were not estimated PTSD $=$ posttraumatic stress disorder; PTSD negative shifts $=$ PTSD negative shifts in cognitions and mood; $\alpha_{1-4}=$ proportional change parameters; $\gamma_{1-4}=$ cross-lagged effects. 
second (4-8 months posttrauma) and third (8-12 months posttrauma) time intervals, on average, exhibited greater decreases (or smaller increases) in alcohol consumption over the subsequent time intervals (8-12 and 12-16 months, respectively, posttrauma). Similarly, the second PTSD to change in alcohol use cross-lagged effect was significant and negative in the PTSD total severity and negative shifts in cognitions and mood with alcohol use models. There were no significant cross-lagged effects for the PTSD avoidance model. Parameter estimates for the susceptibility theory PTSD intrusion with alcohol use model are displayed in Table 34; there were no significant cross-lagged coupling effects.

For all of the PTSD with drug use frequency pairings, models representing the susceptibility theory resulted in a statistically poorer fit to the data as compared to models representing the self-medication theory (Table 29). Parameter estimates for the self-medication theory PTSD with drug use models are contained in Tables 35 to 39. Significance of parameter estimates were comparable to the abovementioned PTSD with drug use BLDS models contained in Tables 24 through 28. In terms of the cross-lagged coupling effects, the third PTSD to change in drug use cross-lagged effect was significant and negative in all drug use models. As such, participants with higher PTSD scores between 8 and 12 months were expected to have greater decreases (or smaller increases) in the frequency of drug use over the 12 to 16 months time interval.

Self-medication versus mutual maintenance theory models: Hypothesis $3 d$. When PTSD with alcohol use BLDS models reflecting the mutual maintenance theory (i.e., all cross lagged effects were estimated; mutual maintenance theory PTSD with alcohol use models are those contained in Tables 19-23) and the self-medication theory (i.e., cross lagged effects from alcohol use to change in PTSD were constrained to 0) were compared, models representing the 
Table 34

Susceptibility Theory Bivariate Latent Difference Score Model for PTSD Intrusion Cluster Severity with Alcohol Use Frequency

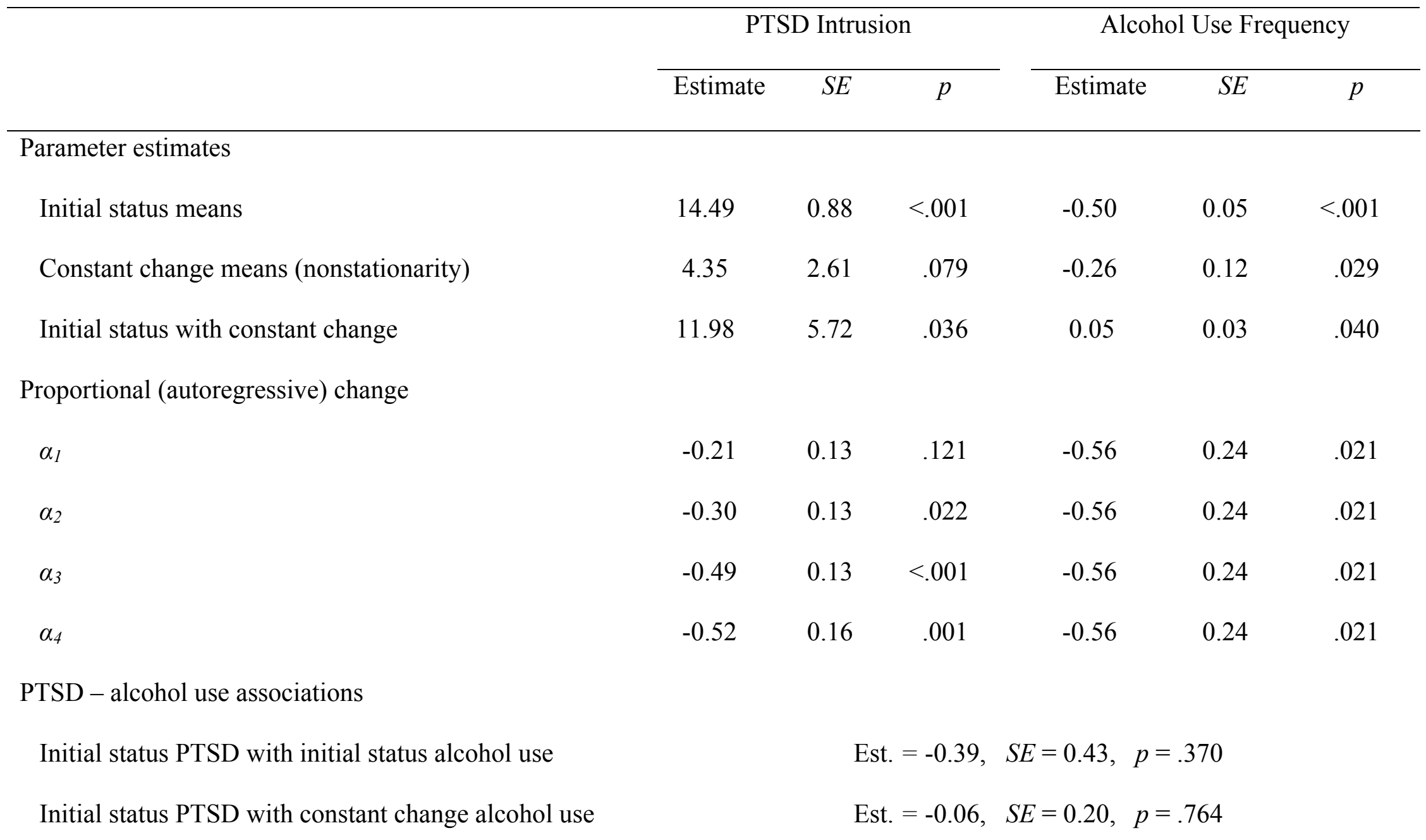


Constant change PTSD with initial status alcohol use

PTSD - alcohol use cross-lagged effects

\begin{tabular}{|c|c|c|c|c|c|c|}
\hline$\gamma_{1}$ & 9.84 & 5.28 & .062 & -- & -- & -- \\
\hline$\gamma_{2}$ & 7.50 & 5.20 & .149 & -- & -- & -- \\
\hline$\gamma_{3}$ & 5.45 & 5.01 & .276 & -- & -- & -- \\
\hline$\gamma_{4}$ & 6.56 & 5.09 & .197 & -- & -- & -- \\
\hline Error variance & 14.71 & 1.53 & $<.001$ & 0.05 & 0.01 & $<.001$ \\
\hline
\end{tabular}

Note. Model consisted of pairing of the dual variable proportional latent difference score (LDS) PTSD intrusion cluster severity and the dual fixed proportional change LDS alcohol use frequency models, with cross lagged paths from PTSD to change in alcohol use constrained to 0 to reflect the susceptibility theory. "--" denotes parameters that were not estimated. PTSD = posttraumatic stress disorder; $\alpha_{1-4}=$ proportional change parameters; $\gamma_{1-4}=$ cross-lagged effects. 
Table 35

Self-Medication Theory Bivariate Latent Difference Score Model for PTSD Total Symptom Severity with Drug Use Frequency

\begin{tabular}{|c|c|c|c|c|c|c|}
\hline & \multicolumn{3}{|c|}{ PTSD Total Severity } & \multicolumn{3}{|c|}{ Drug Use Frequency } \\
\hline & Estimate & $S E$ & $p$ & Estimate & $S E$ & $p$ \\
\hline \multicolumn{7}{|l|}{ Parameter estimates } \\
\hline Initial status means & 56.61 & 3.33 & $<.001$ & -0.21 & 0.04 & $<.001$ \\
\hline Constant change means (nonstationarity) & 10.75 & 3.65 & .003 & -- & -- & -- \\
\hline Initial status with constant change & 235.46 & 63.34 & $<.001$ & -- & -- & -- \\
\hline \multicolumn{7}{|l|}{ Proportional (autoregressive) change } \\
\hline$\alpha_{1}$ & -0.37 & 0.07 & $<.001$ & -0.04 & 0.03 & .127 \\
\hline$\alpha_{2}$ & -0.52 & 0.08 & $<.001$ & -0.04 & 0.03 & .127 \\
\hline$\alpha_{3}$ & -0.50 & 0.10 & $<.001$ & -0.04 & 0.03 & .127 \\
\hline$\alpha_{4}$ & -0.68 & 0.11 & $<.001$ & -0.04 & 0.03 & .127 \\
\hline \multicolumn{7}{|l|}{ PTSD - drug use cross-lagged effects } \\
\hline$\gamma_{1}$ & -- & -- & -- & $<-0.01$ & $<0.01$ & .322 \\
\hline$\gamma_{2}$ & -- & -- & -- & $<0.01$ & $<0.01$ & .157 \\
\hline
\end{tabular}




$\begin{array}{lll}-- & -- & - \\ -- & -- & -\end{array}$

$$
<-0.01
$$

Note. Model consisted of pairing of the dual variable proportional latent difference score (LDS) PTSD total symptom severity and the fixed proportional change LDS drug use frequency models, with cross lagged paths from drug use to change in PTSD constrained to 0 to reflect the self-medication theory. “--” denotes parameters that were not estimated. PTSD $=$ posttraumatic stress disorder; $\alpha_{1-4}=$ proportional change parameters; $\gamma_{1-4}=$ cross-lagged effects. 
Table 36

Self-Medication Theory Bivariate Latent Difference Score Model for PTSD Intrusion Cluster Severity with Drug Use Frequency

\begin{tabular}{|c|c|c|c|c|c|c|}
\hline & \multicolumn{3}{|c|}{ PTSD Intrusion } & \multicolumn{3}{|c|}{ Drug Use Frequency } \\
\hline & Estimate & $S E$ & $p$ & Estimate & $S E$ & $p$ \\
\hline \multicolumn{7}{|l|}{ Parameter estimates } \\
\hline Initial status means & 14.51 & 0.92 & $<.001$ & -0.21 & 0.04 & $<.001$ \\
\hline Constant change means (nonstationarity) & 2.27 & 1.02 & .025 & -- & -- & -- \\
\hline Initial status with constant change & 17.29 & 5.12 & .001 & -- & -- & -- \\
\hline \multicolumn{7}{|l|}{ Proportional (autoregressive) change } \\
\hline$\alpha_{1}$ & -0.40 & 0.08 & $<.001$ & -0.40 & 0.03 & .179 \\
\hline$\alpha_{2}$ & -0.42 & 0.10 & $<.001$ & -0.40 & 0.03 & .179 \\
\hline$\alpha_{3}$ & -0.57 & 0.11 & $<.001$ & -0.40 & 0.03 & .179 \\
\hline$\alpha_{4}$ & -0.67 & 0.14 & $<.001$ & -0.40 & 0.03 & .179 \\
\hline \multicolumn{7}{|l|}{ PTSD - drug use cross-lagged effects } \\
\hline$\gamma_{1}$ & 2.98 & 2.12 & .160 & $<-0.01$ & $<0.01$ & .297 \\
\hline$\gamma_{2}$ & 2.00 & 1.97 & .311 & $<0.01$ & $<0.01$ & .114 \\
\hline
\end{tabular}




$\begin{array}{lrrrrrr}\gamma_{3} & 1.86 & 1.98 & .349 & -0.01 & <0.01 & .023 \\ \gamma_{4} & 3.30 & 2.77 & .233 & <0.01 & <0.01 & .734 \\ \text { Error variance } & 15.63 & 1.54 & <.001 & 0.04 & <0.01 & <.001\end{array}$

Note. Model consisted of pairing of the dual variable proportional latent difference score (LDS) PTSD intrusion cluster severity and the fixed proportional change LDS drug use frequency models, with cross lagged paths from drug use to change in PTSD constrained to 0 to reflect the self-medication theory. "--" denotes parameters that were not estimated; “--" denotes parameters that were not estimate due to the absence of the constant change component for drug use. PTSD = posttraumatic stress disorder; $\alpha_{1-4}=$ proportional change parameters; $\gamma_{1-4}=$ cross-lagged effects. 
Table 37

Self-Medication Theory Bivariate Latent Difference Score Model for PTSD Hyperarousal Cluster Severity with Drug Use Frequency

\begin{tabular}{|c|c|c|c|c|c|c|}
\hline & \multicolumn{3}{|c|}{ PTSD Hyperarousal } & \multicolumn{3}{|c|}{ Drug Use Frequency } \\
\hline & Estimate & $S E$ & $p$ & Estimate & $S E$ & $p$ \\
\hline \multicolumn{7}{|l|}{ Parameter estimates } \\
\hline Initial status means & 15.07 & 1.08 & $<.001$ & -0.20 & 0.04 & $<.001$ \\
\hline Constant change means (nonstationarity) & 2.39 & 1.20 & .047 & -- & -- & -- \\
\hline Initial status with constant change & 16.59 & 6.16 & .007 & -- & -- & -- \\
\hline \multicolumn{7}{|l|}{ Proportional (autoregressive) change } \\
\hline$\alpha_{1}$ & -0.30 & 0.09 & .001 & -0.04 & 0.03 & .124 \\
\hline$\alpha_{2}$ & -0.50 & 0.10 & $<.001$ & -0.04 & 0.03 & .124 \\
\hline$\alpha_{3}$ & -0.50 & 0.13 & $<.001$ & -0.04 & 0.03 & .124 \\
\hline$\alpha_{4}$ & -0.62 & 0.14 & $<.001$ & -0.04 & 0.03 & .124 \\
\hline \multicolumn{7}{|l|}{ PTSD - drug use cross-lagged effects } \\
\hline$\gamma_{1}$ & -- & -- & -- & $<-0.01$ & $<0.01$ & .179 \\
\hline$\gamma_{2}$ & -- & -- & -- & $<0.01$ & $<0.01$ & .214 \\
\hline
\end{tabular}


$\gamma_{4}$

Error variance

$<.001$

$-0.01$

$<0.01$

.032

Note. Model consisted of pairing of the dual variable proportional latent difference score (LDS) PTSD hyperarousal cluster severity and the fixed proportional change LDS drug use frequency models, with cross lagged paths from drug use to change in PTSD constrained to 0 to reflect the self-medication theory. "--" denotes parameters that were not estimate due to the absence of the constant change component for drug use. PTSD = posttraumatic stress disorder; $\alpha_{1-4}=$ proportional change parameters; $\gamma_{1-4}=$ cross-lagged effects. 
Table 38

Self-Medication Theory Bivariate Latent Difference Score Model for PTSD Avoidance Cluster Severity with Drug Use Frequency

\begin{tabular}{|c|c|c|c|c|c|c|}
\hline & \multicolumn{3}{|c|}{ PTSD Avoidance } & \multicolumn{3}{|c|}{ Drug Use Frequency } \\
\hline & Estimate & $S E$ & $p$ & Estimate & $S E$ & $p$ \\
\hline \multicolumn{7}{|l|}{ Parameter estimates } \\
\hline Initial status means & 6.60 & 0.53 & $<.001$ & -0.20 & 0.04 & $<.001$ \\
\hline Constant change means (nonstationarity) & 0.57 & 0.69 & .410 & -- & -- & -- \\
\hline Initial status with constant change & 1.96 & 1.52 & .197 & -- & -- & -- \\
\hline \multicolumn{7}{|l|}{ Proportional (autoregressive) change } \\
\hline$\alpha_{1}$ & -0.25 & 0.12 & .036 & -0.05 & 0.03 & .077 \\
\hline$\alpha_{2}$ & -0.34 & 0.14 & .016 & -0.05 & 0.03 & .077 \\
\hline$\alpha_{3}$ & -0.39 & 0.16 & .017 & -0.05 & 0.03 & .077 \\
\hline$\alpha_{4}$ & -0.64 & 0.18 & $<.001$ & -0.05 & 0.03 & .077 \\
\hline \multicolumn{7}{|l|}{ PTSD - drug use cross-lagged effects } \\
\hline$\gamma_{1}$ & -- & -- & -- & -0.01 & 0.01 & .249 \\
\hline$\gamma_{2}$ & -- & -- & -- & 0.01 & $<0.01$ & .203 \\
\hline
\end{tabular}


Note. Model consisted of pairing of the dual variable proportional latent difference score (LDS) PTSD avoidance cluster severity and the fixed proportional change LDS drug use frequency models, with cross lagged paths from drug use to change in PTSD constrained to 0 to reflect the self-medication theory. "--" denotes parameters that were not estimate due to the absence of the constant change component for drug use. PTSD = posttraumatic stress disorder; $\alpha_{1-4}=$ proportional change parameters; $\gamma_{1-4}=$ cross-lagged effects. 
Table 39

Self-Medication Theory Bivariate Latent Difference Score Model for PTSD Negative Shifts in Cognitions and Mood Cluster Severity with Drug Use Frequency

\begin{tabular}{|c|c|c|c|c|c|c|}
\hline & \multicolumn{3}{|c|}{ PTSD Negative Shifts } & \multicolumn{3}{|c|}{ Drug Use Frequency } \\
\hline & Estimate & $S E$ & $p$ & Estimate & $S E$ & $p$ \\
\hline \multicolumn{7}{|l|}{ Parameter estimates } \\
\hline Initial status means & 16.98 & 1.39 & $<.001$ & -0.21 & 0.04 & $<.001$ \\
\hline Constant change means (nonstationarity) & 3.58 & 1.50 & .017 & -- & -- & -- \\
\hline Initial status with constant change & 37.77 & 11.90 & .002 & -- & -- & -- \\
\hline \multicolumn{7}{|l|}{ Proportional (autoregressive) change } \\
\hline$\alpha_{1}$ & -0.38 & 0.10 & $<.001$ & -0.04 & 0.03 & .143 \\
\hline$\alpha_{2}$ & -0.66 & 0.11 & $<.001$ & -0.04 & 0.03 & .143 \\
\hline$\alpha_{3}$ & -0.51 & 0.16 & .002 & -0.04 & 0.03 & .143 \\
\hline$\alpha_{4}$ & -0.73 & 0.16 & $<.001$ & -0.04 & 0.03 & .143 \\
\hline \multicolumn{7}{|l|}{ PTSD - drug use cross-lagged effects } \\
\hline$\gamma_{1}$ & -- & -- & -- & $<-0.01$ & $<0.01$ & .505 \\
\hline
\end{tabular}




\begin{tabular}{|c|c|c|c|c|c|c|}
\hline$\gamma_{2}$ & -- & -- & -- & $<0.01$ & $<0.01$ & .119 \\
\hline$\gamma_{3}$ & -- & -- & -- & -0.01 & $<0.01$ & .004 \\
\hline$\gamma_{4}$ & -- & -- & -- & $<0.01$ & $<0.01$ & .553 \\
\hline Error variance & 36.82 & 3.60 & $<.001$ & 0.04 & $<0.01$ & $<.001$ \\
\hline
\end{tabular}

Note. Model consisted of pairing of the dual variable proportional latent difference score (LDS) PTSD negative shifts in cognitions and mood cluster severity and the fixed proportional change LDS drug use frequency models, with cross lagged paths from drug use to change in PTSD constrained to 0 to reflect the self-medication theory. "--" denotes parameters that were not estimate due to the absence of the constant change component for drug use. PTSD = posttraumatic stress disorder; PTSD negative shifts $=$ PTSD negative shifts in cognitions and mood; $\alpha_{1-4}=$ proportional change parameters; $\gamma_{1-4}=$ cross-lagged effects. 
mutual maintenance theory resulted in a statistically poorer fit to the data as compared to models representing the self-medication theory. Tables 30 through 33 contain parameter estimates for the self-medication theory models of PTSD with alcohol use involving PTSD total, hyperarousal, avoidance, and negative shifts in cognitions and mood PTSD components. Parameter estimates for the self-medication theory PTSD intrusion with alcohol use BLDS model is contained in Table 40.

With respect to drug use, results indicated that the mutual maintenance theory BLDS models resulted in a statistically poorer fit to the data than the self-medication theory BLDS models for each of the PTSD with drug use pairings (see Table 41). Mutual maintenance theory PTSD with drug use models are contained in Tables 24 to 28, while the self-medication theory PTSD with drug use models are contained in Tables 35-39.

\section{Discussion}

PTSD and SUD commonly co-occur following trauma, and their co-occurrence is associated with substantial costs; however, our understanding of the timing and sequencing of these posttrauma mental health conditions remains in its infancy. This study sought to address a significant gap in the literature by examining the trajectories of PTSD symptoms and substance use among a community sample of individuals recently exposed to a traumatic event, with a focus on the potential moderating roles of PTSD and SUD diagnoses at the final assessment. Additionally, while several prominent theories have been proposed to explain the phenomenon of comorbid PTSD symptoms and substance use posttrauma, research findings are mixed. Thus, in attempt to better understand the functional relationship between PTSD symptoms and substance use posttrauma, this study compared models reflecting the theories of self-medication, susceptibility, and mutual maintenance. 
Table 40

Self-Medication Theory Bivariate Latent Difference Score Model for PTSD Intrusion Cluster Severity with Alcohol Use Frequency

\begin{tabular}{|c|c|c|c|c|c|c|}
\hline & \multicolumn{3}{|c|}{ PTSD Intrusion } & \multicolumn{3}{|c|}{ Alcohol Use Frequency } \\
\hline & Estimate & $S E$ & $p$ & Estimate & $S E$ & $p$ \\
\hline \multicolumn{7}{|l|}{ Parameter estimates } \\
\hline Initial status means & 14.47 & 0.90 & $<.001$ & -0.52 & 0.05 & $<.001$ \\
\hline Constant change means (nonstationarity) & 2.33 & 1.06 & .027 & -0.13 & 0.13 & .292 \\
\hline Initial status with constant change & 17.72 & 5.15 & .001 & 0.04 & 0.03 & .110 \\
\hline \multicolumn{7}{|l|}{ Proportional (autoregressive) change } \\
\hline$\alpha_{1}$ & -0.40 & 0.08 & $<.001$ & -0.48 & 0.24 & .044 \\
\hline$\alpha_{2}$ & -0.43 & 0.10 & $<.001$ & -0.48 & 0.24 & .044 \\
\hline$\alpha_{3}$ & -0.58 & 0.12 & $<.001$ & -0.48 & 0.24 & .044 \\
\hline$\alpha_{4}$ & -0.68 & 0.14 & $<.001$ & -0.48 & 0.24 & .044 \\
\hline \multicolumn{7}{|l|}{ PTSD - alcohol use associations } \\
\hline Initial status PTSD with initial status alcohol use & \multicolumn{6}{|c|}{ Est. $=-0.32, \quad S E=0.44, p=.469$} \\
\hline Initial status PTSD with constant change alcohol use & \multicolumn{6}{|c|}{ Est. $=0.43, S E=0.33, p=.190$} \\
\hline
\end{tabular}


Constant change PTSD with initial status alcohol use

PTSD - alcohol use cross-lagged effects

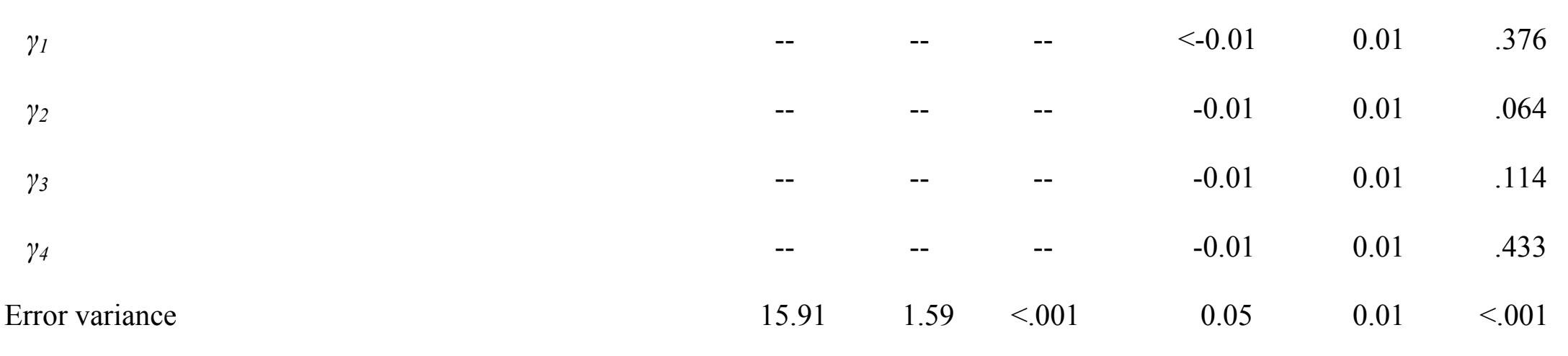

Note. Model consisted of pairing of the dual variable proportional latent difference score (LDS) PTSD intrusion cluster severity and the dual fixed proportional change LDS alcohol use frequency models, with cross lagged paths from alcohol use to PTSD constrained to 0 to reflect the self-medication theory. “--" denotes parameters that were not estimated. PTSD = posttraumatic stress disorder; $\alpha_{1-4}=$ proportional change parameters; $\gamma_{1-4}=$ cross-lagged effects.

Est. $=0.14, S E=0.21, p=.522$

\section{4} the dual fixed proportional change LDS alcohol use frequency models, with cross lagged paths from alcohol use to PTSD constrained proportional change parameters; $\gamma_{1-4}$ cross-lagged effects. 
Table 41

Goodness of Fit Estimates for Self-Medication Theory and Mutual Maintenance Theory Bivariate Latent Difference Score Models

\section{Alcohol Use Dual Fixed Change with PTSD (component) Dual Variable Proportional Change}

\begin{tabular}{|c|c|c|c|c|c|c|c|c|c|c|}
\hline PTSD Model: & $\chi^{2}(d f)$ & $\chi^{2} / d f$ & $p$ & CFI & RMSEA $(90 \% \mathrm{CI})$ & PCLOSE & SRMR & $\mathrm{AIC}$ & $\chi^{2} \Delta$ & $p$ \\
\hline Total Severity (SMed) & $55.13(40)$ & 1.38 & .056 & 0.97 & $0.05(0.00,0.08)$ & .424 & 0.06 & 4392.48 & 2.12 & .714 \\
\hline Total Severity (Bidir) & $53.01(36)$ & 1.47 & 0.03 & 0.97 & $0.06(0.02,0.09)$ & .314 & 0.06 & 4398.35 & - & - \\
\hline Intrusion (SMed) & $51.17(40)$ & 1.28 & .111 & 0.98 & $0.05(0.00,0.08)$ & .560 & 0.06 & 3259.17 & 7.63 & .106 \\
\hline Intrusion (Bidir) & $43.54(36)$ & 1.21 & .181 & 0.99 & $0.04(0.00,0.08)$ & .646 & 0.06 & 3259.54 & - & - \\
\hline Hyperarousal (SMed) & $56.57(40)$ & 1.41 & .043 & 0.97 & $0.06(0.01,0.09)$ & .377 & 0.07 & 3398.58 & 0.65 & .957 \\
\hline Hyperarousal (Bidir) & $55.92(36)$ & 1.55 & .018 & 0.96 & $0.06(0.03,0.10)$ & .233 & 0.07 & 3405.93 & - & - \\
\hline Avoidance (SMed) & $41.85(40)$ & 1.05 & .390 & 1.00 & $0.02(0.00,0.06)$ & .849 & .07 & 2836.17 & 2.54 & .637 \\
\hline Avoidance (Bidir) & $39.31(36)$ & 1.09 & .324 & 0.99 & $0.03(0.00,0.07)$ & .788 & 0.06 & 2841.62 & - & - \\
\hline Negative Shifts (SMed) & $63.10(40)$ & 1.58 & .011 & 0.95 & $0.07(0.03,0.09)$ & .202 & .071 & 3626.57 & 1.52 & .823 \\
\hline Negative Shifts (Bidir) & $61.58(36)$ & 1.71 & .010 & 0.95 & $0.07(0.04,0.10)$ & .119 & 0.07 & 3633.05 & $\ldots$ & \\
\hline
\end{tabular}


Drug Use Fixed Proportional Change with PTSD (component) Dual Variable Proportional Change

\begin{tabular}{|c|c|c|c|c|c|c|c|c|c|c|}
\hline PTSD Model: & $\chi^{2}(d f)$ & $\chi^{2} / d f$ & $p$ & $\mathrm{CFI}$ & RMSEA $(90 \% \mathrm{CI})$ & PCLOSE & SRMR & AIC & $\chi^{2} \Delta$ & $p$ \\
\hline Total Severity (SMed) & $59.65(47)$ & 1.27 & .102 & 0.98 & $0.04(0.00,0.08)$ & .585 & 0.07 & 4253.89 & 1.32 & .858 \\
\hline Total Severity (Bidir) & $58.33(43)$ & 1.36 & .059 & 0.97 & $0.05(0.00,0.08)$ & .454 & 0.07 & 4260.56 & - & - \\
\hline Intrusion (SMed) & $48.59(47)$ & 1.03 & .409 & 1.00 & $0.02(0.00,0.06)$ & .882 & 0.08 & 3120.38 & 3.12 & .538 \\
\hline Intrusion (Bidir) & $45.47(43)$ & 1.06 & .370 & 1.00 & $0.02(0.00,0.06)$ & .848 & 0.06 & 3125.26 & - & - \\
\hline Hyperarousal (SMed) & $57.84(47)$ & 1.23 & .134 & 0.98 & $0.04(0.00,0.07)$ & .642 & 0.08 & 3265.40 & 1.99 & .738 \\
\hline Hyperarousal (Bidir) & $55.85(43)$ & 1.30 & .090 & 0.97 & $0.05(0.00,0.08)$ & .536 & 0.08 & 3271.42 & - & - \\
\hline Avoidance (Bidir) & $47.55(43)$ & 1.11 & .293 & 0.99 & $0.03(0.00,0.07)$ & .797 & 0.07 & 2700.32 & - & - \\
\hline Negative Shifts (SMed) & $64.29(47)$ & 1.37 & .048 & 0.96 & $0.05(0.01,0.08)$ & .437 & 0.07 & 3485.24 & 0.44 & .979 \\
\hline Negative Shifts (Bidir) & $63.85(43)$ & 1.48 & .021 & 0.96 & $0.06(0.02,0.09)$ & .288 & 0.07 & 3492.80 & - & - \\
\hline
\end{tabular}

Note. $\mathrm{PTSD}=$ posttraumatic stress disorder; SMed = Model reflecting self-medication theory (i.e., cross-lagged coupling effects from PTSD to substance use were estimated while constraining to 0 the cross-lagged coupling effects from substance use to PTSD); Bidir $=$ Model reflecting bidirectional temporal relations between PTSD and substance use (i.e., all cross-lagged coupling effects were 
estimated); Negative Shifts = negative shifts in cognitions and mood PTSD symptom cluster; $\chi^{2}=$ Chi Square statistic; CFI =

Comparative Fit Index; RMSEA = Root Mean Square Error of Approximation along with 90\% confidence intervals; PCLOSE $=p$ of Close Fit; SRMR = Standardized Root Mean Square Residual; AIC = Akaike Information Criterion; $\chi^{2} \Delta=$ Chi Square difference statistic of self-medication model relative to the mutual maintenance model. 
In summary, the severity of PTSD symptoms decreased over time following trauma, and having a diagnosis of PTSD at the final assessment moderated this trajectory such that PTSD symptom severity did not improve among individuals with end-state PTSD. Initial PTSD symptom severity was also higher among those with a PTSD diagnosis compared to those without PTSD. In contrast, the influence of SUD appeared to be negligible. Regarding substance use, the relationship between alcohol use and end-state diagnostic status did not reach significance. Both PTSD and SUD diagnoses were associated with drug use frequency, however, and PTSD diagnosis had a small positive effect on drug use across time. In terms of temporal relationships between PTSD symptoms and substance use posttrauma, significant and negative PTSD to change in substance use cross-lagged paths were found across most of the BLDS models, while only one significant substance use to change in PTSD severity cross-lagged path emerged in the PTSD intrusion with alcohol use BLDS model. PTSD total severity with alcohol use and drug use models were mostly consistent with their respective PTSD cluster models.

\section{Aim 1: Trajectory of PTSD Symptom Severity Posttrauma}

Consistent with the extant literature (Riggs et al., 1995; Rothbaum et al., 1992), PTSD symptom severity decreased over time for the overall sample. Additionally, when the trajectory of symptom severity was considered as a function of PTSD diagnosis at the fourth assessment, only those individuals with no end-state PTSD evidenced a decline in PTSD symptoms; symptoms for those with PTSD at end-state persisted over time. These results are in line with prior research and the conceptualization of PTSD as a disorder of impeded recovery (Blanchard et al., 1996). For instance, Shalev, Peri, Canetti, and Schreiber (1996) investigated PTSD symptom severity among trauma survivors who were admitted to hospital for a minimum of 2 days due to physical injury resulting from traumatic exposure; assessments were conducted at 1- 
week and 6-months posttrauma. Based on the Impact of Events Scale (IES; Horowitz, Wilner, \& Alvarez, 1979), total PTSD symptom severity increased from the first to second assessment for those with, and decreased for those without, a PTSD diagnosis at 6 months.

Rothbaum et al. (1992) assessed female sexual assault victims shortly after trauma exposure (first assessment was completed an average of 13 days posttrauma), followed by weekly assessments of PTSD symptoms for the subsequent 12 weeks. PTSD severity was based on interview questions pertaining to re-experiencing, numbing, and arousal symptoms of PTSD, in addition to the avoidance subscale of the IES, that collectively corresponded to DSM-III-R criteria for PTSD (APA, 1987). Sexual assault victims who did not meet diagnostic criteria for PTSD at the final assessment evidenced an overall improvement in PTSD severity across the assessment period. However, while individuals with PTSD at the final assessment showed some reduction in PTSD severity by the fourth assessment, PTSD symptoms were found to remain stable from the fourth to final assessment.

Some research has underscored problematic substance use as a potential impediment to natural recovery from PTSD symptoms posttrauma (Acierno et al., 1999; Matar et al., 2009; McCauley et al., 2009). In particular, a diagnosis of SUD prior to trauma exposure has been associated with higher severity of PTSD symptomatology (Kaysen et al., 2006; 2011; McFarlane, 2009). Findings pertaining to SUD increasing risk for subsequent diagnosis of PTSD, on the other hand, are mixed (e.g., Acierno et al., 1999; Blanchard et al., 1996; McFarlane et al., 2009). Accordingly, it was anticipated that end-state diagnostic status would moderate the course of PTSD such that symptoms would evidence the least decline among those with PTSD/SUD, a moderate decline among those with PTSD-only, and the most decline among those with SUD-only or neither disorder. These hypotheses were mostly supported. 
The trajectory of PTSD severity did have the steepest decline among participants with neither disorder at the final assessment and those with an end-state diagnosis of SUD-only. Indeed, as can be seen in Figure 5 Panel A, the slopes associated with SUD-only and neither disorder were comparable with symptoms decreasing across time. In addition and as noted earlier, PTSD symptoms persisted across the assessment period among individuals with end-state PTSD-only. Counter to expectation, however, end-state PTSD-only was not associated with a steeper decline in PTSD severity as compared to PTSD/SUD. Rather, these slopes appeared similarly flat. When taken together, these results suggest that PTSD diagnosis moderates the trajectory of PTSD severity irrespective of presence or absence of end-state SUD.

This finding appears inconsistent with studies that have reported severity of PTSD symptoms as higher among individuals with PTSD/SUD as compared to those with PTSD in absence of co-occurring SUD (e.g., Saladin et al., 1995). For instance, Blanco and colleagues (2013) investigated differences in PTSD symptom presentation for individuals with PTSD/AUD compared to PTSD-only. These authors used Wave 2 of the National Epidemiological Survey on Alcohol and Related Conditions to explore differences among individuals with lifetime PTSD and AUD $(n=597)$ compared to lifetime PTSD in absence of lifetime AUD $(n=2,463)$. Individual with PTSD/AUD endorsed more PTSD symptom criteria. In another study, AUD at time of assault was found to moderate the course of PTSD symptoms in the 6 months following trauma among a community sample of women, with the AUD group demonstrating a shallower PTSD severity slope across time (i.e., less improvement) than the group without AUD at time of assault (Kaysen et al., 2011).

It is possible that the lack of significant difference in the present study is an artifact of the data and, particularly, attributable to insufficient power to detect effects. Although a small 
proportion of individuals met diagnostic status for PTSD-only and SUD-only at the final assessment ( $n=10$ and $n=14$, respectively), only 2 individuals endorsed symptoms consistent with co-occurring PTSD/SUD. Relatedly, although not statistically significant, it is notable that there was a small and positive effect $(d=0.21)$ of end-state SUD on initial PTSD symptom severity that is in line with prior work. Kaysen and colleagues (2006) explored the impact of lifetime AUD on PTSD symptoms posttrauma in a sample of female crime victims. PTSD symptoms and AUD diagnostic status were measured with the CAPS and Structured Interview for DSM-III-R (Spitzer, Williams, Gibbon, \& First, 1989), respectively, at two time points: 2-4 weeks and 3 months postassault. While women with lifetime AUD had higher PTSD scores at both assessments, they evidenced the same pattern of PTSD symptom recovery as women without lifetime AUD. Thus, it may be that SUD influences the initial severity but not the trajectory of PTSD symptoms following trauma.

An additional reason for the unexpected findings could relate to the timing of assessments. More specifically, it could be that SUD at the time of trauma exposure increases risk for severity and chronicity of PTSD symptoms but has a weaker association with PTSD over time. In other words, although SUD may increase risk for impeded natural recovery of PTSD in the immediate aftermath of trauma, the initial severity and posttrauma course of PTSD symptoms may look similar among individuals who ultimately receive diagnoses of PTSD-only and PTSD/SUD in the months and years following trauma. Most studies that have investigated the role of SUD on PTSD symptoms subsequent to trauma examined SUD diagnosis more proximal to the time of trauma exposure (e.g., Kaysen et al., 2006; 2011; McFarlane et al., 2009), whereas this study examined SUD at the final assessment that was an average of 513 days following trauma. To this author's knowledge, no prior studies have examined the trajectory of PTSD 
symptoms based on end-state SUD diagnostic status. However, partial support for this notion may be found in studies looking at differences in symptom severity among individuals with chronic PTSD. One such study involved comparison of baseline characteristics of two groups of treatment-seeking individuals - those with PTSD/SUD and those with PTSD in absence of SUD (Drapkin et al., 2011). Data comparing these two groups were drawn from two randomized controlled trials, one related to PTSD treatment (Rothbaum et al., 2006) and the other related to PTSD/AUD treatment (Foa \& Williams, 2010). Although PTSD/AUD was associated with poorer psychosocial functioning at pretreatment (i.e., more likely to be unemployment, have lower income, fewer with a college education, less likely to live with a significant other) as compared to the PTSD-only group, these groups did not differ with respect to PTSD symptom severity (Drapkin et al., 2011).

Taken together, results pertaining to Aim 1 are consistent with the broader literature underscoring PTSD as a disorder of impeded recovery; individuals without PTSD at end-state, regardless of the presence of SUD, recovered from PTSD symptoms in the months following trauma. In contrast to prior work, the initial severity and trajectory of PTSD symptoms did not differ based on end-state PTSD/SUD compared to PTSD-only. While this finding is consistent with some research, the majority of prior studies have demonstrated higher severity and chronicity of PTSD symptoms among those with co-occurring SUD. Additional research is needed to clarify whether individuals who meet diagnostic criteria for PTSD/SUD in the months following trauma exposure have a more chronic or comparable course of PTSD symptoms than individuals without co-occurring SUD.

\section{Aim 2: Trajectories of Alcohol and Drug Use Posttrauma}

Although much is known about the course of PTSD symptoms posttrauma, a comparative 
lack of research has been conducted on changes in substance use following traumatic event exposure. Still fewer studies have focused on the course of substance use as a function of PTSD and SUD diagnostic status in the months following trauma. It is generally considered, however, that trauma exposure is associated with increased substance use (Dewart et al., 2006; DiMaggio et al., 2009; Grieger, Fullerton, \& Ursano, 2003; Stein et al., 2004; Vlahov et al., 2002). Interestingly, results of the current study found that substance use frequency, overall, did not change as a function of time.

This unexpected finding could be attributable to methodological differences across studies. Prior research has often focused on the initial months following trauma, whereas the first assessment for this study was an average of 127 days subsequent to trauma exposure. Indeed, studies in this area have often been conducted within the first 3 months (Stein et al., 2004; Vlahov et al., 2002) or 6 months following trauma (Hassin, Keyes, Hatzenbuehler, Aharonovich, \& Alderson, 2007; Pfefferbaum \& Doughty, 2001; Simons, Gaher, Jacobs, Meyer, \& JohnsonJimenez, 2005). Relatedly, frequency of initial substance use following trauma in prior studies has been based on the first assessment. On the contrary, this study modeled the data by specifying the intercept (i.e., initial frequency of use) as the date of traumatic event exposure, which was done to provide an estimate of substance use frequency immediately following trauma. However, this estimate is based on the trajectory of substance use data acquired from the four assessments completed by participants. Thus, results may not accurately reflect potential changes in substance use more proximal to trauma exposure. While it is yet unknown, it is possible that substance use varies within the first several months of trauma exposure then generally returns to pretrauma levels or otherwise becomes consistent across time. 
Additionally, the lack of significant change in the overall slopes for alcohol and drug use may be a result of systematic differences in the manifestation of substance use following trauma. For instance, Simons and colleagues (2005) investigated alcohol use among 779 Red Cross workers who responded to the September 11,2001, terrorist attacks 6 months postattack. Participants were asked about their frequency of alcohol use in the prior 6 months, as well as perceived changes in use in the month following their disaster response. The vast majority of the sample (90\%) reported no change in alcohol consumption, $3 \%$ reported an increase in alcohol use, and $6 \%$ reported a decrease. Moreover, these percentages were similar when eliminating nondrinkers ( $86 \%, 9 \%$, and 5\%, respectively). Accordingly, individual differences in increased versus decreased substance use posttrauma could have been lost when looking at trajectory of substance use for the overall sample.

Another factor that has been implicated in the literature as being associated with frequency of substance use posttrauma is mental health diagnostic status - in particular, the presence of PTSD and SUD. Among a sample of 75 patients who were in treatment for SUD on or after September 11, 2001, 55\% of those who relapsed attributed their substance use to distress resulting from the terrorist attacks (Dewart et al., 2006). Relatedly, PTSD was found to significantly predict increased alcohol consumption among individuals seeking supportive therapy and crisis intervention in response to the Oklahoma City federal building bombing, even when controlling for physical exposure and injury (Pfefferbaum \& Doughty, 2001). While it is notable that there was a small and positive effect size for the relationship between PTSD and the course of drug use in the current study $(d=0.29)$, neither end-state PTSD nor SUD were significantly associated with the trajectory of alcohol or drug use over time. 
As abovementioned, one potential explanation for the unexpected findings may be due to elapsed time between date of traumatic event and the first assessment. A study conducted by Hassin and colleagues (2007) partially supports this notion with respect to SUD. In this longitudinal study, 791 drinkers living in close proximity to the World Trade Center completed three assessments including a baseline assessment (1991-1992), a 1-year follow-up assessment, and a 10-year follow-up assessment in 2002 subsequent to the September 11 terrorist attacks. During this 10-year follow-up, participants were asked to report on the maximum number of drinks consumed the week after the terrorist attacks and for the 16 weeks following the attacks (September 11 through to the end of 2001 year). Although a history of AUD was associated with higher number of drinks consumed in the week following September 11, AUD was not significantly associated with number of drinks consumed in the 16 weeks following the attacks.

There are also additional methodological considerations that may have resulted in discrepant findings as compared to prior research. First, this study used end-state SUD when investigating both alcohol consumption and drug use across time, rather than looking at end-state alcohol use disorder in relation to alcohol consumption and end-state drug use disorder in relation to drug use. It was determined to use end-state SUD in order to remain consistent with analyses pertaining to the trajectory of PTSD symptom severity, as well as in consideration of power to detect associations given low endorsement of end-state SUD across the sample. Yet, it appears reasonable to expect that alcohol use would be more strongly associated with AUD than nonalcohol-related SUD, and vice versa. Second, the majority of research pertaining to substance use subsequent to trauma comes from population-based data or samples that are otherwise not explicitly restricted to trauma exposed persons, such as studies looking at man-made or natural disasters (e.g., Dewart et al., 2006; Vlahov et al., 2002). In contrast, all individuals in the current 
study were trauma-exposed. While the pervasive effects of terrorist attacks and other large-scale disasters may influence subsequent substance use, it is not possible to accurately account for PTSD symptomatology and resulting diagnosis if persons were at arm's length of the disaster and did not experience a Criterion A traumatic event. Some authors have even cautioned that change in alcohol consumption in response to disaster may be overestimated due to recall and attribution bias (Nordløkken et al., 2013).

Indeed, the results of the current study may accurately reflect relationships between substance use and PTSD and SUD when considering trauma-exposed persons. For instance, Gould and colleagues (2011) assessed current and lifetime mental health disorders using the Structured Clinical Interview for DSM-IV Disorders (First, Spitzer, Gibbon, \& Williams, 2002), among a sample of 102 adults who recently experienced a traumatic brain injury. Patients also completed follow-up assessments at 3-, 6-, and 12-month postinjury. SUD was the most frequently endorsed lifetime disorder. Despite significantly more individuals meeting criteria for a mental health disorder in the year following injury, fewer patients had SUD at the final assessment (from $34 \%$ at postinjury to $12 \%$ at 1 -year follow-up). While the authors encouraged caution when interpreting the findings as a 1-year period of abstinence was recommended by treating doctors due to traumatic brain injury (Gould et al., 2011), other studies have documented a similar (lack of) relationship between substance use and PTSD and SUD (North et al., 2002; North, Kawasaki, Spitznagel, \& Hong, 2004). Along these same lines, no differences were found in mean number of drug use days among heroin and cocaine users before and after the September 11, 2001, terrorist attacks (Factor et al., 2002; Weiss et al., 2002), and alcohol consumption was reported as comparable across PTSD/SUD and PTSD without SUD treatment seeking samples (Drapkin et al., 2011). 


\section{Aim 3: Temporal Sequencing of PTSD Symptom Severity and Substance Use Posttrauma}

The third aim of this study investigated temporal associations between PTSD symptoms and substance use posttrauma. While substantial support has accrued for the self-medication theory of comorbid PTSD/SUD, the majority of this literature is based on cross sectional designs or longitudinal research that is unidirectional in focus (i.e., exploring the effect of PTSD on substance use or vice versa). Moreover, the few longitudinal studies that have investigated bidirectional relationships between PTSD and substance use have yielded equivocal findings (Langdon et al., 2016; Nickerson et al., 2014; Read et al., 2013). Thus, this study aimed to address a substantive gap in the PTSD/SUD literature.

To investigate the directionality of associations between posttrauma substance use and PTSD, PTSD symptom cluster and substance use outcome variables were represented by singleindicator latent variables within the BLDS models as per convention (King et al., 2006a; Nickerson et al., 2014). However, for total PTSD symptom severity a single-indicator LDS model was compared to a four-indicator LDS model to determine which latent factor structure for PTSD demonstrated a better fit to the data. Given prior work documenting a four-factor model to best capture the dimensional nature of PTSD (Asmundson et al., 2000; Gentes et al., 2014; 2015; King, Leskin, King, \& Weathers, 1998) and in recognition that a four-factor model corresponds to mechanisms theorized to underlie PTSD (Foa, Zinbarg, \& Rothbaum, 1992; Foa, Riggs, \& Gershuny, 1995), it was hypothesized that the four-indicator model would best fit the data. In contrast to expectation, the single-indicator dual variable change LDS model demonstrated as best fitting the data.

It is notable that recommended procedures for SEM suggest that measurement models be fit to the data prior to incorporating the structural model. However, this approach was not used in 
the current study because it would have required further restrictions placed on the singleindicator model (Little, 2013) that would not have corresponded with conventional LDS modeling approaches for single-indicator latent variables (King et al., 2009; McArdle \& Hamagami, 2001). As such, the single-indicator and four-indicator LDS models were compared at the structural level, which may explain the discrepant results as compared to prior literature on the factor structure of PTSD. Moreover, it is likely that model complexity coupled with sample size contributed to poorer fit for the four-indicator latent variable models. Replication of these findings with a larger sample is recommended.

With respect to direction of association between PTSD and substance use, results largely supported the notion of PTSD symptom severity as a potential driver of subsequent change in substance use frequency. Specifically, in most of the BLDS models fit to the data, at least one of the PTSD to change in substance use cross-lagged paths were significant, while only one of the substance use to change in PTSD severity paths was significant across all BLDS models. Furthermore, with the exception of the PTSD intrusion with alcohol use model, the models depicting mutual maintenance (i.e., cross-lagged paths from PTSD to substance use and substance use to PTSD were estimated) and susceptibility (i.e., cross-lagged paths from substance use to PTSD were estimated and cross-lagged paths from PTSD to substance use were constrained to 0 ) theories resulted in a poorer fit to the data as compared to those depicting the self-medication theory (i.e., cross-lagged paths from PTSD to substance use were estimated and cross-lagged paths from substance use to PTSD were constrained to 0). However, rather than higher PTSD scores at one time point being associated with greater increases (or smaller decreases) in substance use over the subsequent time interval as might be expected based on the 
self-medication theory, the opposite relationship was found: Higher PTSD severity was associated with subsequent decreases (or smaller increases) in substance use.

This finding stands in contrast to the bulk of the literature related to PTSD/SUD that supports a self-medication pathway between PTSD symptoms and substance use and substancerelated problems (Back et al., 2006a; 2006b; Gaher et al., 2014; Hien et al., 2010; Ouimette et al., 2010; Simpson et al., 2014; Wolitzky-Taylor et al., 2012), as well as studies documenting support for the susceptibility hypothesis (Coffey et al., 2007; Kaysen et al., 2011). Positive relationships between PTSD symptoms and subsequent alcohol use problems have also been demonstrated through statistical application of BLDS modeling. Langdon and colleagues (2016) administered self-report measures of PTSD symptoms and alcohol problems to veterans within one year of returning from deployment and again approximately 7 months later. Using procedures similar to those outlined in this dissertation, data were assigned to three time classes that were a mean of 230, 419, and 644 days since returning from deployment. As would be anticipated through the lens of self-medication, significant and positive cross-lagged effects were found from PTSD to change in alcohol problems in the PTSD total, emotional numbing, and intrusion cluster models.

Inability to measure substance-related problems coupled with the approximate 4-month interval between data points in this study likely contributed to the unexpected findings. While associations between substance-related problems and PTSD may remain stable over the course of months, increased frequency of substance use may reflect a response to PTSD symptoms that is more proximal in nature and susceptible to short-term fluctuations in PTSD symptoms. This interpretation is consistent with a growing literature that has highlighted the importance of investigating bidirectional relationships between PTSD and substance use within close proximity 
of one another (Gaher et al., 2014; Kaysen et al., 2014; Simpson et al., 2014). Results from studies investigating daily PTSD symptoms and substance use have reported increased PTSD symptoms as related to increased subsequent alcohol consumption (Gaher et al., 2014; Simpson et al., 2014), with some suggestion that PTSD symptoms may differ in their association with substance use. For instance, Kaysen and colleagues (2014) found that women drank more on days when they experienced higher intrusive and avoidance symptoms but less on days when they experienced higher dysphoric symptoms of PTSD (conceptualized as loss of interest, emotional numbing, irritability, sleep disturbance, feeling detached from others, concentration problems, and foreshortened future). Daily monitoring protocols may be particularly useful in uncovering a more nuanced understanding of the interplay between PTSD and substance use.

Discrepant findings may be attributable, at least in part, to differences between samples. As prior mentioned, much of the research suggestive of increased substance use following trauma is based on samples that are not restricted to individuals who have experienced a Criterion A event or PTSD symptoms that are not tied to a particular trauma (e.g., Dewart et al., 2006; Gaher et al., 2014; Langdon et al., 2016; Vlahov et al., 2002), while others are drawn from samples of individuals in treatment for PTSD or SUD (e.g., Back et al., 2006a; 2006b; Hien et al., 2010; Ouimette et al., 2010). Regarding treatment-involved samples with PTSD/SUD, the interplay between PTSD symptoms and substance use may be qualitatively different among treatment-engaged individuals compared to a community-based sample with recent trauma exposure. More specifically, there could be a potentially greater proportion of individuals who have an established pattern of substance use to alleviate PTSD symptoms in comorbid treatmentseeking samples. Additionally, substance use withdrawal and PTSD symptoms overlap extensively (Jacobsen et al., 2001; Stewart \& Conrod, 2003); as such, decreases in substance use 
and SUD symptoms would be expected to coincide with decreased PTSD symptoms during treatment. In the case of research looking at deployment- and disaster-related traumas where PTSD symptoms are not tied to an established Criterion A event, perhaps these studies are capturing the greater impact that large-scale events can have on other domains of functioning (e.g., transition to civilian life, loss of stable housing, financial instability, relationship discord), which have also been associated with problematic substance use (e.g., Jacobson et al., 2008; Whisman \& Uebelacker, 2006). Alternatively, differential risk for PTSD based on different types of traumatic events has been well established, with interpersonal and combat-related trauma being associated with greater prevalence of PTSD and severity of symptoms (Frans et al., 2005; Kessler et al., 1995; Wanklyn et al., in press). It follows that type of trauma may also be differentially related to substance use posttrauma. Thus, it is likely that inclusion of individuals with a range of traumas in the present study obscured relationships between PTSD and substance use and contributed to the inconsistent findings.

It is noteworthy that most prior work has focused on alcohol use in industrialized countries where alcohol consumption is pervasive and socially normative. While this author could find no research on the topic, it is conceivable that during times of shared distress, such as the immediate wake of terrorist attacks and natural disasters, individuals seek out increased social support; depending on the social network, increased social interaction could inadvertently involve increased occasions to consume alcohol. Perhaps an absence of a shared trauma coupled with a longer duration of follow-up in this study attenuated the association between substance use and posttrauma sequelae widely observed in prior research.

Of relevance, PTSD symptoms have been associated with decreased substance use in past research (Gould et al., 2011; Sbordone, Liter, \& Pettler-Jennings, 1995; Simons et al., 2005), 
including an investigation of bidirectional relationships between PTSD symptoms and alcohol problems following hospital admission due to traumatic injury (Nickerson et al., 2014). Participants completed an initial assessment and three follow-up assessments at 3-, 12-, and 24months postinjury, each of which included the CAPS to determine PTSD cluster severity and the AUDIT as a measure of alcohol-related problems. Based on BLDS modeling, there were significant and positive cross-lagged paths from PTSD symptoms at 12 months to subsequent change in alcohol problems between 12 and 24 months for the re-experiencing, avoidance, and hyperarousal cluster models in support of the self-medication hypothesis. Interestingly, for both the emotional numbing and hyperarousal models, greater severity of PTSD symptoms at the initial assessment was associated with decreased alcohol problems at the following assessment. Thus, Nickerson and colleagues' (2014) results partially accord with the finding of the present study, where higher severity of PTSD symptoms at either the second or third time interval were associated with greater decreases (or smaller increases) in substance use across all alcohol and drug use models except the PTSD avoidance with alcohol use model.

From the perspective that PTSD symptoms and alcohol-related behaviours demonstrate components of both symptom change and stability (e.g., Bonanno \& Mancini, 2012; Del Boca, Darkes, Greenbaum, \& Goldman, 2004), Read and colleagues (2013) tested a novel application of trait-state-error modeling in the relation between PTSD and alcohol use. College students who endorsed a Criterion A traumatic event and past month drinking at the first assessment were followed over the course of 3 years, with assessments occurring 4 times per year. Interestingly, high trait PTSD symptoms were associated with low levels of trait alcohol consumption, while state components of PTSD symptoms and alcohol use demonstrated bidirectional cross-lagged associations consistent with the mutual maintenance theory (i.e., greater PTSD symptoms 
predicted greater subsequent alcohol use and vice versa). Further, more variance for both PTSD and substance use was accounted for by the trait component of the model. Building from this, future research may benefit from attention to both state and trait components of PTSD and substance use to disentangle inconsistent findings relating to direction of association between these constructs.

\section{Limitations and Future Directions}

This study has a number of limitations that are important to acknowledge, most notably the time at which assessments were conducted relative to trauma exposure. The larger study from which these data were derived investigated risk and resilience factors in the course of PTSD and posttrauma recovery with an emphasis on better understanding interpersonal factors that contribute to change in PTSD symptoms following trauma. Based on prior research suggesting that associations between PTSD symptoms and interpersonal factors vary up to 2 years following trauma (e.g., Kaniasty \& Norris, 2008), and in attempt to balance feasibility of recruiting individuals following trauma, an enrolment window of 6 months posttrauma was utilized. This resulted in participants in this study completing the first assessment an average of 127 days posttrauma. Yet, prior research has demonstrated significant change in PTSD severity occurring within the first 3 months following trauma exposure (Riggs et al., 1995; Rothbaum et al., 1992). As such, it is possible that important information was lost pertaining to the trajectories of PTSD severity and substance use following trauma, as well as the temporal ordering of associations between these constructs. Future studies could benefit from completing the initial assessment closer to the date of traumatic exposure to inform patterns of temporal change during the initial weeks and months following trauma. Where feasible, it may be particularly useful to incorporate a daily monitoring protocol within a longer-term follow-up study. In this vein, data could be 
collected for a period of 5 days on four different occasions over the course of a year, permitting investigation of both distal and proximal relationships between PTSD symptoms and substance use and related problems.

Although the limited inclusion and exclusion criteria for this study can be viewed as a strength with respect to generalizability of the findings, the heterogeneity of the sample is also a limitation. Associations between PTSD symptoms and substance use may be stronger among persons with more severe PTSD symptoms; therefore research focusing on individuals with greater distress may better lend itself to investigation of competing models of co-occurring PTSD and substance use. At a minimum, future research should consider restricting recruitment to individuals who endorse recent drug use or problematic levels of alcohol consumption. For instance, moderate levels of posttrauma alcohol consumption have been identified as predictive of lower levels of PTSD severity compared to minimal or problematic levels of alcohol use (Maes, Delmeire, Mylle, \& Altamura, 2001; McFarlane et al., 2009). If individuals who denied recent alcohol use were excluded from the current study, it may have been possible to investigate differences across level of substance use.

An alternative approach to offset heterogeneity could have involved recruiting a larger sample. While the current sample size was adequate to perform analyses central to this study, it precluded the ability to investigate additional variables that have been implicated as related to PTSD and substance use. Examination of moderators may have allowed for a more comprehensive understanding of posttrauma substance use and associations with PTSD. Promising directions for future investigation might consider the impact of social support, social interaction and social network norms with respect to substance use, impulsivity, participant perception of reasons for use, and exposure to varied and multiple types of traumatic events. For 
example, the benefits of social support have been well documented in the PTSD literature (Brewin, Andrews, \& Valentine, 2000; Ozer, Best, Lipsey, \& Weiss, 2003) and this construct may moderate the association between PTSD and substance use. While speculative, trauma may result in some individuals spending more time with supportive others that could result in increased drinking occasions; in this scenario, failing to account for social support could give the appearance that increased alcohol is associated with decreased PTSD severity. Similarly, increased isolation due to PTSD symptoms may lead to less drinking among a subpopulation of individuals who typically drink in social settings. This reduction in social support could in turn exacerbate PTSD severity, which, if not assessed, could be interpreted as increased PTSD severity being associated with reductions in alcohol use.

Further in regards to social support, although this dissertation included all traumaexposed individuals from the larger study irrespective of whether they completed alone or as part of a dyad, it is possible that the sample for this dissertation was comprised of individuals with stronger social support as compared to prior studies. While recruitment materials were targeted to individuals and did not mention participation with a significant other, interested persons who contacted study staff were informed that participation required completion of the study with a close other aware of the traumatic event. Individuals who indicated that they did not have or were unwilling to include a close other were given 48 hours to try and identify a close other with whom to complete the study; only after this 48 -hour period when recontacted by study staff were individuals invited to participate in the study alone if they remained unable or unwilling to enroll with a close other. A number of participants were unreachable at this time or otherwise indicated that they were no longer interested in completing the study. It is possible that, for at least some individuals, this decision was influenced by a lack of close other to invite or individuals' not 
wanting to disclose their trauma to someone else. If this conjecture was accurate, it may follow that individuals in this sample were less symptomatic than prior studies given increased perceived social support (Brewin et al., 2000; Ozer et al., 2003) and disclosure of traumatic event to supportive others (e.g., Ullman, 2007) are associated with lower PTSD symptoms.

The longitudinal examination of PTSD symptoms in relationship to drug use makes a valuable contribution to the literature as most work has focused on alcohol use. However, given low rates of drug use reported within this sample, it was not possible to run models separated by drug class. While insufficient attention has been paid to relationships between PTSD and drug use, a number of studies suggest that different drug classes may be differentially related to PTSD clusters (e.g., Avant, Davis, \& Cranston, 2011; Tull, Gratz, Aklin, \& Lejuez, 2010; Villagonzalo et al., 2011). As such, future examination of the differential associations between PTSD symptoms and drug classes is warranted.

Additional limitations include not controlling for the potential effects of treatment utilization, not assessing for substance use problems across time for BLDS analysis, and using a single measurement assessment for each construct of interest. To this author's knowledge, this study is the first to explore associations between substance use and DSM-5 PTSD symptoms in a longitudinal design, which is a significant study strength. Given participant recruitment commenced prior to the release of the CAPS for DSM-5, the CAPS for DSM-IV was modified by including additional items to account for PTSD symptoms as established in the DSM-5. As such, it is important that future studies replicate this work with clinician-administered and selfreport measures of PTSD designed to measure DSM-5 criteria. Also, this study extends the literature by incorporating a prospective longitudinal design; yet, this design does not permit causal inference. An exciting area of future work would be investigation of the interplay between 
PTSD symptoms and substance use using laboratory-based experimental design. Trauma-cue induction studies have been valuable in providing a more nuanced understanding of alcohol cravings in the context of PTSD symptoms (Coffey et al., 2010; Coffey, Stasiewicz, Hughes, \& Brimo, 2006; Nosen et al., 2012). Extending this work by looking at self-administration of alcohol, rather than craving, subsequent to trauma cue induction could further clarify temporal relationships between PTSD and substance use.

\section{Conclusion and Clinical Implications}

Limitations notwithstanding, this study provides a valuable contribution to the literature on co-occurring PTSD/SUD and highlights potential research directions for further understanding of symptom interplay between these two disorders. Though replication is needed, these results suggest that the trajectory of PTSD symptom severity is comparable for individuals with PTSD in absence of SUD and those with co-occurring PTSD/SUD; thus strengthening the growing body of research demonstrating trauma-focused treatment is feasible for this population. While PTSD treatment has often been considered as contraindicated for individuals with SUD, the findings of this study coupled with a burgeoning literature inclusive of well-controlled randomized controlled trials suggest trauma-focused comorbid treatment options should be made available to individuals presenting with PTSD/SUD. Additionally, the unexpected relationship between greater PTSD symptom severity and subsequent decreases in substance use may further

underscore the complex relationship between PTSD and substance use over time. Examination of potential mediators and moderators of the relation between PTSD and SUD is recommended as a critical focus for future research. 


\section{References}

Acierno, R., Resnick, H., Kilpatrick, D. G., Sauders, B., \& Best, C. L. (1999). Risk factors for rape, physical assault, and posttraumatic stress disorder in women: Examination of differential multivariate relationships. Journal of Anxiety Disorders, 13, 541-563. doi:10.1016/S0887-6185(99)00030-4

Afifi, T. O., Henriksen,C. A., Asmundson, G. J. G., \& Sareen, J. (2012). Victimization and perpetration of intimate partner violence and substance use disorders in a nationally representative sample. Journal of Nervous and Mental Disease, 200, 684-691. doi:10.1097/NMD.0b013e3182613f64

American Psychiatric Association. (1987). Diagnostic and statistical manual of mental disorders (3rd ed., rev.). Arlington, VA: Author.

American Psychiatric Association. (2000). Diagnostic and statistical manual of mental disorders (4th ed., text rev.). Arlington, VA: Author.

American Psychiatric Association. (2013). Diagnostic and statistical manual of mental disorders (5th ed.). Arlington, VA: Author.

Armony, J. L., \& LeDoux, J. E. (1997). How the brain processes emotional information. In R. Yehuda \& A. C. McFarlane (Eds.), Psychobiology of posttraumatic stress disorder (pp. 259-270). New York, NY: New York Academy of Sciences.

Aronson, K. (2003). Alcohol: A recently identified risk factor for breast cancer. Canadian Medical Association Journal, 168, 1147-1148.

Asmundson, G. J. G., Frombach, I., McQuaid, J., Pedrelli, P., Lenox, R., \& Stein, M. B. (2000). Dimensionality of posttraumatic stress symptoms: A confirmatory factor analysis of DSM-IV symptom clusters and other symptom models. Behaviour Research and 
Therapy, 38, 203-214.

Avant, E. A., Davis, J. L., \& Cranston, C. C. (2011). Posttraumatic stress symptom clusters, trauma history, and substance use among college students. Journal of Aggression, Maltreatment \& Trauma, 20, 539-555. doi:10.1080/10926771.2011.588153

Babor, T., Fuente, J., Saunders, J., \& Grant, M. (1989). The Alcohol Use Disorders Identification Test: Guidelines for use in primary health care. Geneva, Switzerland: Division of Mental Health, World Health Organization.

Back, S., Dansky, B.S., Coffey, S. F., Saladin, M. E., Sonne, S., \& Brady, K. T. (2000). Cocaine dependence with and without post-traumatic stress disorder: A comparison of substance use, trauma history and psychiatric comorbidity. American Journal on Addictions, 9, 5162. doi:10.1080/10550490050172227

Back, S. E., Brady, K. T., Jaanimägi, U., \& Jackson, J. L. (2006a). Cocaine dependence and PTSD: A pilot study of symptom interplay and treatment preferences. Addictive Behaviors, 31, 351-354. doi:10.1016/j.addbeh.2005.05.008

Back, S. E., Brady, K. T., Sonne, S. C., \& Verduin, M. L. (2006b). Symptom improvement in cooccurring PTSD and alcohol dependence. Journal of Nervous and Mental Disease, 194, 690-696. doi:10.1097/01.nmd.0000235794.12794.8a

Bagnardi, V., Blangiardo, M., La Vecchia, C., \& Corrao, G. (2001). A meta-analysis of alcohol drinking and cancer risk. British Journal of Cancer, 85, 1700-1705. doi:10.1054/bjoc.2001.2140

Bates, M. E., Bowden, S. C., \& Barry, D. (2002). Neurocognitive impairment associated with alcohol use disorders: Implications for treatment. Experimental and Clinical Psychopharmacology, 10, 193-212. doi:10.1037//1064-1297.10.3.193 
Bentler, P. M. (1990). Comparative fit indexes in structural models. Psychological Bulletin, 88, 588-606. doi:10.1037/0033-2909.88.3.588

Blake, D. D., Weathers, F. W., Nagy, L. M., Kaloupek, D. G., Gusman, F. D., Charney, D. S., \& Keane, T. M. (1995). The development of a clinician-administered PTSD scale. Journal of Traumatic Stress, 8, 75-90. doi:10.1002/jts.2490080106

Blanchard, E. B., Hickling, E. J., Barton, K. A., Taylor, A. E., Loos, W. R., \& Jones-Alexander, J. (1996). One-year prospective follow-up of motor vehicle accident victims. Behaviour Research and Therapy, 34, 775-786.

Blanco, C., Xu, Y., Brady, K., Pérez-Fuentes, G., Okuda, M., \& Wang, S. (2013). Comorbidity of posttraumatic stress disorder with alcohol dependence among US adults: Results from National Epidemiological Survey on Alcohol and Related Conditions. Drug and Alcohol Dependence, 132, 630-638. doi:10.1016/j.drugalcdep.2013.04.016

Bollen, K. (1989). Structural equations with latent variables. Hoboken, NJ: John Wiley \& Sons.

Bolton, J., Cox, B., Clara, I., \& Sareen, J. (2006). Use of alcohol and drugs to self-medicate anxiety disorders in a nationally representative sample. Journal of Nervous and Mental Disease, 194, 818-825. doi:10.1097/01.nmd.0000244481.63148.98

Bonanno, G. A., \& Mancini, A. D. (2012). Beyond resilience and PTSD: Mapping the heterogeneity of responses to potential trauma. Psychological Trauma: Theory, Research, Practice, and Policy, 4, 74-83. doi:10.1037/a0017829

Boscarino, J. A. (2006). Posttraumatic stress disorder and mortality among U.S. army veterans 30 years after military service. Annals of Epidemiology, 16, 248-256. doi:10.1016/j.annepidem.2005.03.009

Brady, K. T., Back, S. E., \& Coffey, S. F. (2004). Substance abuse and posttraumatic stress 
disorder. Current Directions in Psychological Science, 13, 206-209. doi:10.1111/j.09637214.2004.00309.x

Brady, K. T., \& Sinha, R. (2005). Co-occurring mental and substance use disorders: The neurobiological effects of chronic stress. American Journal of Psychiatry, 162, 14831493. doi:10.1176/appi.ajp.162.8.1483

Brewin, C. R., Andrews, B., \& Valentine, J. D. (2000). Meta-analysis of risk factors for posttraumatic stress disorder in trauma-exposed adults. Journal of Consulting and Clinical Psychology, 68, 748-766. doi:10.1037/0022-006X.68.5.748

Browne, M. W., \& Cudeck, R. (1993). Alternative ways of assessing model fit. In K. A. Bollen \& J. S. Long (Eds.), Testing structural equation models (pp. 136-162). Thousand Oaks, CA: Sage.

Bremner, J. D., Southwick, S. M., Darnell, A., \& Charney, D. S. (1996). Chronic PTSD in Vietnam combat veterans: Course of illness and substance abuse. American Journal of Psychiatry, 153, 369-375.

Byrne, B. M. (2010). Structural equation modeling with AMOS: Basic concepts, applications, and programming (2nd ed.). New York, NY: Routledge.

Cahalan, D., Cisin, I. H., \& Crossley, H. M. (1969). American drinking practices: A national study of drinking behavior and attitudes. New Brunswick, NJ: Rutgers Center of Alcohol Studies.

Cerdá, M., Tracy, M., \& Galea, S. (2011). Prospective population based study of changes in alcohol use and binge drinking after a mass traumatic event. Drug and Alcohol Dependence, 115, 1-8. doi:10.1016/j.drugalcdep.2010.09.011

Chilcoat, H. D., \& Breslau, N. (1998). Investigations of causal pathways between PTSD and 
drug use disorders. Addictive Behaviors, 23, 827-840. doi:10.1016/S03064603(98)00069-0

Chilcoat, H., \& Menard, C. (2003). Epidemiological investigations: Co-morbidity of posttraumatic stress disorder and substance use disorder. In P. Ouimette \& P. Brown (Eds.), Trauma and substance abuse (pp. 9-28). Washington, DC: American Psychological Association.

Coffey, S. F., Schumacher, J. A., Stasiewicz, P. R., Henslee, A. M., Baillie, L. E., \& Landy, N. (2010). Craving and physiological reactivity to trauma and alcohol cues in posttraumatic stress disorder and alcohol dependence. Experimental and Clinical Psychopharmacology, 18, 340-349. doi:10.1037/a0019790

Coffey, S. F., Schumacher, J. A., Brady, K. T., \& Cotton, B. D. (2007). Changes in PTSD symptomatology during acute and protracted alcohol and cocaine abstinence. Drug and Alcohol Dependence, 87, 241-248. doi:10.1016/j.drugalcdep.2006.08.025

Coffey, S. F., Stasiewicz, P. R., Hughes, P. M., \& Brimo, M. L. (2006). Trauma-focused imaginal exposure for individuals with comorbid posttraumatic stress disorder and alcohol dependence: Revealing mechanisms of alcohol craving in a cue reactivity paradigm. Psychology of Addictive Behaviors, 20, 425-435. doi:10.1037/0893164X.20.4.425

Cohen, B. E., Gima, K., Bertenthal, D., Kim, S., Marmar, C. R., \& Seal, K. H. (2010). Mental health diagnoses and utilization of VA non-mental health medical services among returning Iraq and Afghanistan veterans. Journal of General Internal Medicine, 25, 1824. doi:10.1007/s11606-009-1117-3

Cottler, L. B., Compton, W. M., Mager, D., Spitznagel, E. L., \& Janca, A. (1992). Posttraumatic 
stress disorder among substance users from the general population. American Journal of Psychiatry, 149, 664-670.

Del Boca, F. K., Darkes, J., Greenbaum, P. E., \& Goldman, M. S. (2004). Up close and personal: Temporal variability in the drinking of individual college students during their first year. Journal of Consulting and Clinical Psychology, 72, 155-164. doi:10.1037/0022006X.72.2.155

Dewart, T., Blanche, F., \& Schmeidler, J. (2006). The impact of 9/11 on patients in New York City's substance abuse treatment programs. American Journal of Drug and Alcohol Abuse, 32, 665-672. doi:10.1080/00952990600919435

DiMaggio, C., Galea, S., \& Guohua, Li. (2009). Substance use and misuse in the aftermath of terrorism. A Baysian meta-analysis. Addiction, 104, 894-904. doi:10.1111/j.13600443.2009.02526.x

Drapkin, M. L., Yusko, D., Yasinski, C., Oslin, D., Hembree, E. A., \& Foa, E. B. (2011). Baseline functioning among individuals with posttraumatic stress disorder and alcohol dependence. Journal of Substance Abuse Treatment, 41, 186-192. doi:10.1016/j.jsat.2011.02.012

Driessen, M., Schulte, S., Leudecke, C., Schaefer, I., Sutmann, F., Ohlmeier, M.,...TRAUMASStudy Group. (2008). Trauma and PTSD in patients with alcohol, drug, or dual dependence: A multi-center study. Alcoholism: Clinical and Experimental Research, 32, 481-488. doi:10.1111/j.1530-0277.2007.00591.x

Factor, S. H., Wu, Y., Monserrate, J., Edwards, V., Cuevas, Y., Del Vecchio, S., \& Vlahov, D. (2002). Drug use frequency among street-recruited heroin and cocaine users in Harlem and the Bronx before and after September 11, 2001. Journal of Urban Health, 79, 404- 
448.

Ferrer, E., \& McArdle, J. J. (2003). Alternative structural models for multivariate longitudinal data analysis. Structural Equation Modeling, 10, 493-524. doi:10.1207/S15328007SEM1004_1

First, M. B., Spitzer, R. L., Gibbon, M., Williams, J. B. W. (2002). Structured Clinical Interview for DSM-IV-TR Axis I Disorders, Research Version, Non-Patient Edition (SCID-I/NP). New York: Biometrics Research, New York State Psychiatric Institute.

Foa, E. C., Zinbarg, R., \& Rothbaum, B. O. (1992). Uncontrollability and unpredictability in post-traumatic stress disorder: An animal model. Psychological Bulletin, 112, 218-238. doi:10.1037//0033-2909.112.2.218

Foa, E. B., Riggs, D. S., \& Gershuny, B. S. (1995). Arousal, numbing, and intrusion: symptom structure of PTSD following assault. American Journal of Psychiatry, 152, 116-120.

Foa, E. B., \& Rothbaum, B. O. (1998). Treating the trauma of rape: Cognitive-behavioral therapy for PTSD. New York, NY: Guilford Press.

Foa, E. B., \& Tolin, D. F. (2000). Comparison of the PTSD Symptom Scale-Interview Version and the Clinician-Administered PTSD Scale. Journal of Traumatic Stress, 13, 181-191. doi:10.1023/A:1007781909213

Foa, E. B., \& Williams, M. T. (2010). Methodology of a randomized double-blind clinical trial for comorbid posttraumatic stress disorder and alcohol dependence. Mental Health and Substance Use, 3, 131-147.

Foran, H. M., \& O’Leary, K. D. (2008). Alcohol and intimate partner violence: A meta-analytic review. Clinical Psychology Review, 28, 1222-1234. doi:10.1016/j.cpr.2008.05.001

Garson, G. D. (2013) Hierarchical linear modeling: Guide and applications. Thousand Oaks, 


\section{CA: Sage.}

Gaher, R. M., Simons, J. S., Buchkoski, J., Hahn, A. M., Hofman, N. L., \& Hansen, J. (2014). An experience sampling study of PTSD and alcohol-related problems. Psychology of Addictive Behaviors, 28, 1013-1025. doi:10.1037/a0037257

Gentes, E. L., Dennis, P. A., Kimbrel, N. A., Rissling, M. B., Beckham, J. C., VA Mid-Atlantic MIRECC Workgroup, \& Calhoun, P. S. (2014). DSM-5 posttraumatic stress disorder: Factor structure and rates of diagnosis. Journal of Psychiatric Research, 59, 60-67. doi:10.1016/j.jpsychires.2014.08.014

Gentes, E. L., Dennis, P. A., Kimbrel, N. A., Kirby, A. C., Hair, L. P., Beckham, J. C., \& Calhoun, P. S. (2015). Latent factor structure of DSM-5 posttraumatic stress disorder. Psychopathology Review, 2, 17-29. doi:10.5127/pr.035914

Gould, K. R., Ponsford, J. L., Johnston, L., \& Schönberger, M. (2011). The nature, frequency and course of psychiatric disorders in the first year after traumatic brain injury: A prospective study. Psychological Medicine, 41, 2099-2109. doi:10.1017/S003329171100033X

Grieger, T. A., Fullerton, C. S., \& Ursano, R. J. (2003). Posttraumatic stress disorder, alcohol use, and perceived safety after the terrorist attack on the pentagon. Psychiatric Services, $54,1380-1382$.

Hassin, D. S., Keyes, K. M., Hatzenbuehler, M. L., Aharonovich, E. A., \& Alderson, D. (2007). Alcohol consumption and posttraumatic stress after exposure to terrorism: Effects of proximity, loss, and psychiatric history. American Journal of Public Health, 97, 22682275.

Hien, D. A., Jiang, H., Campbell, A. N. C., Hu, M-C., Miele, G. M., Cohen, L. R.,...Nunes, E. 
V. (2010). Do treatment improvements in PTSD severity affect substance use outcomes? A secondary analysis from a randomized clinical trial in NIDS's Clinical Trials Network. American Journal of Psychiatry, 167, 95-101. doi:10.1176/appi.ajp.2009.09091261

Hoge, C. W., Terhakopian, A., Castro, C. A., Messer, S., \& Engel, C. C. (2007). Association of posttraumatic stress disorder with somatic symptoms, health care visits, and absenteeism among Iraq war veterans. American Journal of Psychiatry, 164, 150-153. doi:10.1176/appi.ajp.164.1.150

Horowitz, M. J., Wilner, N., \& Alvarez, W. (1979). Impact of events scale: A measure of subjective stress. Psychosomatic Medicine, 41, 209-218.

Hox, J. J., \& Bechger, T. M. (1998). An introduction to structural equation modeling. Family Science Review, 11, 354-373.

Hu, L.-T., \& Bentler, P. M. (1999). Cutoff criteria for fit indexes in covariance structure analysis: Conventional criteria versus new alternatives. Structural Equation Modeling, 6, 1-55. doi:10.1080/10705519909540118

Jacobsen, L. K., Southwick, S. M., \& Kosten, T. R. (2001). Substance use disorders in patients with posttraumatic stress disorder: A review of the literature. American Journal of Psychiatry, 158, 1184-1190. doi:10.1176/appi.ajp.158.8.1184

Jacobson, I. G., Ryan, M. A. K., Hooper, T. I., Smith, T. C., Amoroso, P. J., Boyko, E. J.,...Bell, N. S. (2008). Alcohol use and alcohol-related problems before and after military deployment. Journal of the American Medical Association, 13, 663-675. doi:10.1001/jama.300.6.663

Jernigan, T. L., \& Ostergaard, A. L. (1995). When alcoholism affects memory functions: MRI of the brain. Alcohol Health and Research World, 19, 104-107. 
Jones, J. E., Hermann, B. P., Barry, J. J., Gilliam, F., Kanner, A. M., \& Meador, K. J. (2005). Clinical assessment of Axis I psychiatric morbidity in chronic epilepsy: A multicenter investigation. Journal of Neuropsychiatry and Clinical Neuroscience, 17, 172-179. doi:10.1176/appi.neuropsych.17.2.172

Jöreskog, K. G., \& Sörbom, D. (1996). LISREL 8: User's reference guide. Chicago, IL: Scientific Software International.

Kaniasty, K., \& Norris, F. H. (2008). Longitudinal linkages between perceived social support and posttraumatic stress symptoms: Sequential roles of social causation and social selection. Journal of Traumatic Stress, 21, 274-281. doi:10.1002/jts.20334

Kaysen, D., Simpson, T., Dillworth, T., Larimer, M. E., Gutner, C., \& Resick, P. A. (2006). Alcohol problems and posttraumatic stress disorder in female crime victims. Journal of Traumatic Stress, 19, 399-403. doi:10.1002/jts.20122

Kaysen, D., Atkins, D. C., Moore, S. A., Lindgren, K. P., Dillworth, T., \& Simpson, T. (2011). Alcohol use, problems, and the course of posttraumatic stress disorder: A prospective study of female crime victims. Journal of Dual Diagnosis, 7, 262-279. doi:10.1080/15504263.2011.620449

Kaysen, D., Atkins, D. C., Simpson, T. L., Stappenbeck, C. A., Blayney, J. A., Lee, C. M., \& Larimer, M. E. (2014). Proximal relationships between PTSD symptoms and drinking among female college students: Results from a daily monitoring study. Psychology of Addictive Behaviors, 28, 62-73. doi:10.1037/a0033588

Keller, M. B., Lavori, P. W., Friedman, B., Nielsen, E., Endicott, J., McDonald-Scott, P., \& Andreasen, N. C. (1987). The Longitudinal Interval Follow-up Evaluation: A comprehensive method for assessing outcome in prospective longitudinal studies. 
Archives of General Psychiatry, 44, 540-548.

doi:10.1001/archpsyc.1987.01800180050009

Kessler, R. C. (2000). Posttraumatic stress disorder: The burden to the individual and to society. Journal of Clinical Psychiatry, 61, 4-41.

Kessler, R. C., Sonnega, A., Bromet, E., Hughes, M., \& Nelson, C. B. (1995). Posttraumatic stress disorder in the national comorbidity survey. Archives of General Psychiatry, 52, 1048-1060. doi:10.1001/archpsyc.1995.03950240066012

Khantzian, E. J. (1985). The self-medication hypothesis of addictive disorders: Focus on heroin and cocaine dependence. American Journal of Psychiatry, 142, 1259-1264.

Kim, H-Y. (2012). Statistical notes for clinical researchers: Assessing normal distribution (2) using skewness and kurtosis. Restorative Dentistry \& Endodontics, 38, 52-54. doi:10.5395/rde.2013.38.1.52

King, D. W., Leskin, G. A., King, L. A., \& Weathers, F. W. (1998). Confirmatory factor analysis of the clinician-administered PTSD scale: Evidence for the dimensionality of posttraumatic stress disorder. Psychological Assessment, 10, 90-96.

King, D. W., King, L. A., McArdle, J. J., Grimm, K., Jones, R. T., \& Ollendick, T. H. (2006a). Characterizing time in longitudinal trauma research. Journal of Traumatic Stress, 19, 205-215. doi:10.1002/jts.20112

King, L. A., King, D. W., McArdle, J. J., Saxe, G. N., Doron-LaMarca, S., \& Orazem, R. J. (2006b). Latent difference score approach to longitudinal trauma research. Journal of Traumatic Stress, 19, 771-785. doi:10.1002/jts.20188

King, D. W., King, L. A., McArdle, J. J., Shalev, A. Y., \& Doron-LaMarca, S. (2009). Sequential temporal dependencies in associations between symptoms of depression and 
posttraumatic stress disorder: An application of bivariate latent difference score structural equation modeling. Multivariate Behavioral Research, 44, 437-464. doi:10.1080/00273170903103308

Kline, R. B. (2011). Principles and practice of structural equation modeling (3rd ed.). New York, NY: Guilford Press.

Kraemer, H. C., Stice, E., Kazdin, A., Offord, D., \& Kupfer, D. (2001). How do risk factors work together? Mediators, moderators, and independent, over-lapping and proxy risk factors. American Journal of Psychiatry, 158, 848-856. doi:10.1176/appi.ajp.158.6.848

Langdon, K. J., Fox, A. B., King, L. A., King, D. W., Eisen, S., \& Vogt, D. (2016). Examination of the dynamic interplay between posttraumatic stress symptoms and alcohol misuse among combat-exposed Operation Enduring Freedom (OEF)/Operation Iraqi Freedom (OIF) veterans. Journal of Affective Disorders, 196, 234-242.

doi:10.1016/j.jad.2016.02.048

Lecrubier, Y., Sheehan, D. V., Weiller, E., Amorim, P., Sheehan, K. H., Janavs, J., \& Dunbar, G. C. (1997). The Mini International Neuropsychiatric Interview (MINI). A short diagnostic structured interview: Reliability and validity according to the CIDI. European Psychiatry, 12, 224-231. doi:10.1016/S0924-9338(97)83296-8

Leeies, M., Pagura, J., Sareen, J., \& Bolton, J. M. (2010). The use of alcohol and drugs to selfmedicate symptoms of posttraumatic stress disorder. Depression and Anxiety, 27, 731736.

Little, T. D. (2013). Longitudinal structural equation modeling. New York, NY: Guilford Press. Maes, M., Delmeire, L., Mylle, J., \& Altamura, C. (2001). Risk and preventive factors of posttraumatic stress disorder (PTSD): Alcohol consumption and intoxication prior to a 
traumatic event diminishes the relative risk to develop PTSD in response to that trauma. Journal of Affective Disorders, 63, 113-121.

Magruder, K. M., Frueh, B. C., Knapp, R. G., Johnson, M. R., Vaughan III, J. A., Carson, T. C.,...Hebert, R. (2004). PTSD symptoms, demographic characteristics, and functional status among veterans treated in VA primary care clinics. Journal of Traumatic Stress, 17, 293-301. doi:10.1023/B:JOTS.0000038477.47249.c8

Mann, R. E., Smart, R. G., \& Govoni, R. (2003). The epidemiology of alcoholic liver disease. Alcohol Research and Health, 27, 209-219.

Matar, M. A., Zohar, J., Kaplan, Z., \& Cohen, H. (2009). Alprazolam treatment immediately after stress exposure interferes with the normal HPA-stress response and increases vulnerability to subsequent stress in an animal model of PTSD. European Neuropsychopharmacology, 19, 283-295. doi:10.1016/j.euroneuro.2008.12.004

McArdle, J. J. (2001). A latent difference score approach to longitudinal dynamic structural analyses. In R. Cudeck, S. DuToit, \& D. Sorbom (Eds.), Structural equation modeling: Present and future (pp. 342-380). Lincolnwood, IL: Scientific Software International.

McArdle, J. J. (2007). Five steps in the structural factor analysis of longitudinal data. In R. Cudek \& R. MacCallum (Eds.), Factor analysis at 100 years (pp. 99-130). Mahwah, NJ: Lawrence Erlbaum Associates.

McArdle, J. J., \& Hamagami, F. (2001). Latent difference score structural models for linear dynamic analyses with incomplete longitudinal data. In L. Collins (Ed.), New methods for the analysis of change: Decade of behavior (pp. 139-175). Washington, DC: American Psychological Association.

McCauley, J. L., Amstadter, A. B., Danielson, C. K., Ruggiero, K. J., Kilpatrick, D. G., \& 
Resnick, H. S. (2009). Mental health and rape history in relation to non-medical use of prescription drugs in a national sample of women. Addictive Behaviors, 34, 641-648. doi:10.1016/j.addbeh.2009.03.026

McFarlane, A. C. (1998). Epidemiological evidence about the relationship between PTSD and alcohol abuse: The nature of the association. Addictive Behaviors, 23, 813-825. doi:10.1016/S0306-4603(98)00098-7

McFarlane, A. C., Browne, D., Bryant, R. A., O’Donnell, M., Silove, D., Creamer, M., \& Horsley, K. (2009). A longitudinal analysis of alcohol consumption and the risk of posttraumatic symptoms. Journal of Affective Disorders, 118, 166-172. doi:10.1016/j.jad.2009.01.017

Merrill, J. E., \& Read, J. P. (2010). Motivational pathways to unique types of alcohol consequences. Psychology of Addictive Behaviors, 24, 705-711. doi:10.1037/a0020135

Mills, K. L., Teesson, M., Back, S. E., Brady, K. T., Baker, A. L., Hopwood, S.,...Ewer, P. L. (2012). Integrated exposure-based therapy for co-occurring posttraumatic stress disorder and substance dependence: A randomized controlled trial. Journal of the American Medical Association, 308, 690-699.

Monson, C. M., \& Fredman, S. J. (2012). Cognitive-behavioral conjoint therapy for PTSD: Harnessing the healing power of relationships. New York, NY: Guilford Press.

Monson, C. M., Schnurr, P. P., Resick, P. A., Friedman, M. J., Young-Xu, Y., \& Stevens, S. P. (2006). Cognitive processing therapy for veterans with military-related posttraumatic stress disorder. Journal of Consulting and Clinical Psychology, 74, 898-907. doi:10.1037/0022-006X.74.5.898 
Muthén, L. K., \& Muthén, B. O. (1998-2013). Mplus user's guide (7th ed.). Los Angeles, CA: Author.

Najavits, L. M., Harned, M. S., Gallop, R. J., Butler, S. F., Barber, J. P., Thase, M. E., CritsChristoph, P. (2007). Six-month treatment outcomes of cocaine-dependent patients with and without PTSD in a multisite national trial. Journal of Studies on Alcohol and Drugs, $68,353-361$

Nickerson, A., Barnes, J. B., Creamer, M., Forbes, D., McFarlane, A. C., O’Donnell, M.,...Bryant, R. A. (2014). The temporal relationship between posttraumatic stress disorder and problem alcohol use following traumatic injury. Journal of Abnormal Psychology, 123, 821-834. doi:10.1037/a0037920

Nordløkken, A., Pape, H., Wentzel-Larsen, T., \& Heir, T. (2013). Changes in alcohol consumption after a natural disaster: A study of Norwegian survivors after the 2004 Southeast Asia tsunami. BMC Public Health, 13:58. doi.101186/1471-2458-13-58

Norman, S. B., Tate, S. R., Anderson, K. G., \& Brown, S. A. (2007). Do trauma history and PTSD symptoms influence addiction relapse context? Drug and Alcohol Dependence, 90, 89-96. doi:10.1016/j.drugalcdep.2007.03.002

North, C. S., Tivis, L., McMillen, J. C., Pfefferbaum, B., Spitznagel, E. L., Cox, J.,...Smith, E. M. (2002). Psychiatric disorders in rescue workers after the Oklahoma City bombing. American Journal of Psychiatry, 159, 857-859.

North, C. S., Kawasaki, A., Spitznagel, E. L., \& Hong, B. A. (2004). The course of PTSD, major depression, substance abuse, and somatization after a natural disaster. Journal of Nervous and Mental Disease, 192, 823-829. doi:10.1097/01.nmd.0000146911.52616.22 
Nosen, E., Nillni, Y. I., Berenz, E. C., Schumacher, J. A., Stasiewicz, P. R., \& Coffey, S. F. (2012). Cue-elicited affect and craving: Advancement of the conceptualization of craving in co-occurring posttraumatic stress disorder and alcohol dependence. Behavior Modification, 36, 808-833. doi:10.1177/0145445512446741

Orth, U., Berking, M., Walker, N., Meier, L. L., \& Znoj, H. (2008). Forgiveness and psychological adjustment following interpersonal transgressions: A longitudinal analysis. Journal of Research in Personality, 42, 365-385. doi:10.1016/j.jrp.2007.07.003

Ouimette, P. C., Brown, P. J., \& Najavits, L. M. (1998). Course and treatment of patients with both substance use and posttraumatic stress disorders. Addictive Behaviors, 23, 785-795. doi:10.1016/S0306-4603(98)00064-1

Ouimette, P., Goodwin, E., \& Brown, P. J. (2006). Health and well being of substance use disorder patients with and without posttraumatic stress disorder. Addictive Behaviors, 31, 14115-1423. doi:10.1016/j.addbeh.2005.11.010

Ouimette, P., Read, J., \& Brown, P. J. (2005). Consistency of retrospective reports of DSM-IV criterion A traumatic stressors among substance use disorder patients. Journal of Traumatic Stress, 18, 43-51. doi:10.1002/jts.20009

Ouimette, P., Read, J. P., Wade, M., \& Tirone, V. (2010). Modeling associations between posttraumatic stress symptoms and substance use. Addictive Behaviors, 35, 64-67. doi:10.1016/j.addbeh.2009.08.009

Parsons, O. A. (1998). Neurocognitive deficits in alcoholics and social drinkers: A continuum? Alcohol: Clinical and Experimental Research, 22, 954-961. doi:10.1111/j.15300277.1998.tb03895.x

Piselli, M., Elisei, S., Murgia, N., Quartesan, R., \& Abram, K. M. (2009). Co-occurring 
psychiatric and substance use disorders among male detainees in Italy. International Journal of Law and Psychiatry, 32, 101-107. doi:10.1016/j.ijlp.2009.01.006

Pfefferbaum, B., \& Doughty, D. E. (2001). Increased alcohol use in a treatment sample of Oklahoma City bombing victims. Psychiatry, 64, 296-303.

Powers, M. B., Halpern, J. M., Ferenschak, M. P., Gillihan, S. J., \& Foa, E. B. (2010). A metaanalytic review of prolonged exposure for posttraumatic stress disorder. Clinical Psychology Review, 30, 635-641. doi:10.1016/j.cpr.2010.04.007

Qualtrics. (2014). Qualtrics. Provo, UT.

Raudenbush, S. W., \& Bryk, A. S. (2002). Hierarchical linear models: Application and data analysis methods. Thousand Oaks, CA: Sage.

Raudenbush, S. W., Bryk, A. S, \& Congdon, R. (2011). HLM 7 for Windows [Computer software]. Skokie, IL: Scientific Software International, Inc.

Read, J. P., Brown, P. J., \& Kahler, C. W. (2004). Substance use and posttraumatic stress disorders: Symptom interplay and effects on outcome. Addictive Behaviors, 29, 16651672. doi:10.1016/j.addbeh.2004.02.061

Read, J. P., Wardell, J. D., \& Colder, C. R. (2013). Reciprocal associations between PTSD symptoms and alcohol involvement in college: A three-year trait-state-error analysis. Journal of Abnormal Psychology, 122, 984-997. doi:10.1037/a0034918

Rehm, J., Baliunas, D., Brochu, S., Fischer, B., Patra, J., Popova, S.,...Taylor, B. (2006). The cost of substance abuse in Canada 2002. Ottawa, ON: Canadian Centre on Substance Abuse.

Rehm, J., Gmel, G., Sempos, C. T., \& Trevisan, M. (2003). Alcohol-related morbidity and mortality. Alcohol Research and Health, 27, 39-51. 
Resnick, S. G., \& Rosenheck, R. A. (2008). Posttraumatic stress disorder and employment in veterans participating in veterans health administration compensated work therapy. Journal of Rehabilitation Research and Development, 45, 427-436. doi:10.1682/JRRD.2007.06.0093

Richman, J. A., Shannon, C. A., Rospenda, K. M., Flaherty, J. A., \& Fendrich, M. (2009). The relationship between terrorism and distress and drinking: Two years after September 11, 2001. Substance Use \& Misuse, 44, 1665-1680. doi:10.3109/10826080902961989

Riggs, D. S., Rothbaum, B. O., \& Foa, E. B. (1995). A prospective examination of symptoms of posttraumatic stress disorder in victims of nonsexual assault. Journal of Interpersonal Violence, 10, 201-214. doi:10.1177/0886260595010002005

Riggs, D. S., Rukstalis, M., Volpicelli, J. R., Kalmanson, D., \& Foa, E. B. (2003). Demographic and social adjustment characteristics of patients with comorbid posttraumatic stress disorder and alcohol dependence: Potential pitfalls to PTSD treatment. Addictive Behaviors, 28, 1717-1730. doi:10.1016/j.addbeh.2003.08.044

Rodés, J., Salaspuro, M., \& Sorensen, T. J. A. (1999). Alcohol and liver disease. In I. MacDonald (Ed.), Health issues related to alcohol consumption, 2nd ed. (pp.395-450). Oxford, UK: Blackwell Science.

Rothbaum, B. O., Foa, E. B., Riggs, D. S., Murdock, T., \& Walsh, W. (1992). A prospective examination of post-traumatic stress disorder in rape victims. Journal of Traumatic Stress, 5, 455-475. doi:10.1007/BF00977239

Rothbaum, B. O., Cahill, S. P., Foa, E. B., Davidson, J. R., Compton, J., Connor, K. M.,...Hahn, C. G. (2006). Augmentation of sertraline with prolonged exposure in the treatment of posttraumatic stress disorder. Journal of Traumatic Stress, 19, 625-638. 
Saladin, M. E., Brady, K. T., Dansky, B. S., \& Kilpatrick, D. G. (1995). Understanding comorbidity between PTSD and substance use disorders: Two preliminary investigations. Addictive Behaviors, 20, 643-655. doi:10.1016/0306-4603(95)00024-7

Sbordone, R. J., Liter, J. C., \& Pettler-Jennings, P. (1995). Recovery of function following severe traumatic brain injury: A retrospective 10-year follow-up. Brain Injury, 9, 285299.

Schnurr, P. P., \& Green, B. L. (2004). Understanding relationships among trauma, posttraumatic stress disorder, and health outcomes. In P. P. Schnurr \& B. L. Green (Eds.), Trauma and health: Physical health consequences of exposure to extreme stress (pp.247-275). Washington, DC: American Psychological Association.

Schnurr, P. P., Friedman, M. J., Engel, C. C., Foa, E. B., Shea, M. T., Chow, B. K.,... Bernardy, N. (2007). Cognitive behavioral therapy for posttraumatic stress disorder in women: A randomized controlled trial. Journal of the American Medical Association, 297, 820-230. doi:10.1001/jama.297.8.820

Schumacker, R. E., \& Lomax, R. G. (2004). A beginner's guide to structural equation modeling (2nd ed.). New York, NY: Taylor and Francis.

Schumm, J. A., \& Gore, W. L. (2016). Simultaneous treatment of co-occurring posttraumatic stress disorder and substance use disorder. Current Treatment Options in Psychiatry, 3, 28-36. doi:10.1007/s40501-016-0071-z

Shalev, A. Y., Peri, T., Canetti, L., \& Schreiber, S. (1996). Predictors of PTSD in injured trauma survivors: A prospective study. American Journal of Psychiatry, 153, 219-224.

Simons, J. S., Gaher, R. M., Jacobs, G. A., Meyer, D., \& Johnson-Jimenez, E. (2005). Associations between alcohol use and PTSD symptoms among American Red Cross 
disaster relief workers responding to the 9/11/2001 attacks. American Journal of Drug and Alcohol Abuse, 31, 285-304. doi:10.1081/ADA-200047937

Simpson, T. L., Stappenbeck, C. A., Luterek, J. A., Lehavot, K., \& Kaysen, L. D. (2014). Drinking motives moderate daily relationships between PTSD symptoms and alcohol use. Journal of Abnormal Psychology, 123, 237-247. doi:10.1037/a0035193

Sobell, L. C., Maisto, S. A., Sobell, L. C., Cooper, A. M., Cooper, T. C., \& Sanders, B. (1980). Developing a prototype for evaluating alcohol treatment effectiveness. In L. C. Sobel, M. B. Sobell, \& E. Ward (Eds.), Evaluating alcohol and drug treatment effectiveness. New York, NY: Pergamon Press.

Spitzer, R. L., Williams, J. B. W., Gibbon, M., \& First, M. B. (1989). Structured Clinical Interview for DSM-III-R—Non-Patient Version (SCID-MDD). New York, NY: New York State Psychiatric Institute, Biometrics Research.

Stein, B. D., Elliott, M. N., Jaycox, L. H., Collins, R. L., Berry, S. H., Klein, D. J., \& Schuster, M. A. (2004). A national longitudinal study of the psychological consequences of the September 11, 2001 terrorist attacks: Reactions, impairment, and help seeking. Psychiatry, 67, 105-117.

Stewart, S. H. (1996). Alcohol abuse in individuals exposed to trauma: A critical review. Psychological Bulletin, 120, 83-112. doi:10.1037/0033-2909.120.1.83

Stewart, S. H., \& Conrod, P. J. (2003) Psychosocial models of functional associations between posttraumatic stress disorder and substance use disorders. In P. Ouimette, P. J. Brown (Eds.), Trauma and substance abuse (pp. 29-55). Washington DC: American Psychological Association.

Stith, S. M., Smith, D. B., Penn, C. E., Ward, D. B., \& Tritt, D. (2004). Intimate partner physical 
abuse perpetration and victimization risk factors: A meta-analytic review. Aggression and Violent Behavior, 10, 65-98. doi:10.1016/j.avb.2003.09.001

Tabachnick B. C., \& Fidell, L. S. (2007). Using multivariate statistics (5 $5^{\text {th }}$ ed.). Boston, MA: Allyn \& Bacon.

Tate, S. R., Norman, S. B., McQuaid, J. R., \& Brown, S. A. (2007). Health problems of substance-dependent veterans with and those without trauma history. Journal of Substance Abuse Treatment, 33, 25-32. doi:10.1016/j.jsat.2006.11.006

Tull, M. T., Gratz, K. L., Aklin, W. M., \& Lejuez, C. W. (2010). A preliminary examination of the relationships between posttraumatic stress symptoms and crack/cocaine, heroin, and alcohol dependence. Journal of Anxiety Disorders, 24, 55-62. doi:10.1016/j.janxdis.2009.08.006

Ullman, S. E. (2007). Relationship to perpetrator, disclosure, social reactions, and PTSD symptoms in child sexual abuse survivors. Journal of Child Sexual Abuse, 16, 19-36. doi:10.1300/J070v16n01_02

Ullman, S. E., Filipas, H. H., Townsend, S. M., \& Starzynski, L. L. (2006). Correlates of comorbid PTSD and drinking problems among sexual assault survivors. Addictive Behaviors, 31, 128-132. doi:10.1016/j.addbeh.2005.04.002

Van Ameringen, M., Mancini, C., Patterson, B., \& Boyle, M. H. (2008). Post-traumatic stress disorder in Canada. CNS Neuroscience \& Therapeutics, 14, 171-181. doi:10.1111/j.17555949.2008.00049.x

Vasterling, J. J., Schumm, J., Proctor, S. P., Gentry, E., King, D. W., \& King, L. A. (2008). Posttraumatic stress disorder and health functioning in a non-treatment seeking sample of Iraq war veterans: A prospective analysis. Journal of Rehabilitation Research and 
Development, 45, 347-358. doi:10.1682/JRRD.2007.05.0077

Vetter, S., Rossegger, A., Rossler, W., Bisson, J. I., \& Endrass, J. (2008). Exposure to the tsunami disaster, PTSD symptoms and increased substance use - an internet based survey of male and female residents of Switzerland. BMC Public Health, 8, 92. doi:10.1186/1471-2458/8/92

Villagonzalo, K-A, Dodd, S., Ng, F., Mihaly, S., Langbein, A., \& Berk, M. (2011). The relationship between substance use and posttraumatic stress disorder in a methadone maintenance treatment program. Comprehensive Psychiatry, 52, 562-566. doi:10.1016/j.comppsych.2010.10.001

Vlahov, D., Galea, S., Resnick, H., Ahern, J., Boscarino, J. A., Bucuvalas, M.,...Kilpatrick, D. (2002). Increased use of cigarettes, alcohol, and marijuana among Manhattan, New York, residents after the September $11^{\text {th }}$ terrorist attacks. American Journal of Epidemiology, $155,988-996$.

Walker, E. A., Katon, W., Russo, J., Ciechanowski, P., Newman, E., \& Wagner, A. W. (2003). Health care costs associated with posttraumatic stress disorder symptoms in women. Archives of General Psychiatry, 60, 369-374. doi:10.1001/archpsyc.60.4.369

Wanklyn, S. G., Pukay-Martin, N. D., Belus, J. M., St. Cyr, K., Girard, T. A., \& Monson, C. M. (in press). Trauma types as differential predictors of PTSD, MDD and their comorbidity. Canadian Journal of Behavioural Science.

Weathers, F. W., Keane, T. M., \& Davidson, J. R. (2001). Clinician-Administered PTSD Scale: A review of the first ten years of research. Depression and Anxiety, 13, 132-156. doi:10.1002/da.1029 
Weathers, F. W., Ruscio, A. M., \& Keane, T. M. (1999). Psychometric properties of nine scoring rules for the Clinician-Administered Posttraumatic Stress Disorder Scale. Psychological Assessment, 11, 124-133. doi:10.1037/1040- 3590.11.2.124

Whisman, M. A., \& Uebelacker, L. A. (2006). Impairment and distress associated with relationship discord in a national sample of married or cohabiting adults. Journal of Family Psychology, 20, 369-377. doi:10.1037/0893-3200.20.3.369

Willet, J. B., \& Sayer, A. G. (1994). Using covariance structure modeling to detect correlates and predictors of individual change over time. Psychological Bulletin, 116, 363-381. doi:10.1037/0033-2909.116.2.363

Williams, R. H., \& Zimmerman, D. W. (1996). Are simple gain scores obsolete? Applied Psychological Measurement, 20, 59-69. doi:10.1177/014662169602000106

Wolitzky-Taylor, K., Bobova, L., Zinbarg, R. E., Mineka, S., \& Craske, M. G. (2012). Longitudinal investigation of the impact of anxiety and mood disorders in adolescence on subsequent substance use disorder onset and vice versa. Addictive Behaviors, 37, 982985. doi:10.1016/j.addbeh.2012.03.026

Zatzick, D. F., Marmar, C. R., Weiss, D. S., Browner, W. S., Metzler, T., Golding, J. M.,...Wells, K. B. (1997). Posttraumatic stress disorder and functioning and quality of life outcomes in a nationally representative sample of male veterans. American Journal of Psychiatry, 154, 1690-1695.

Zatzick, D. F., Kang, S-M., Muller, H-G., Russo, J. E., Rivara, F. P., Katon, W.,...Roy-Byrne, P. (2002). Predicting posttraumatic distress in hospitalized trauma survivors with acute injuries. American Journal of Psychiatry, 159, 941-946. doi:10.1176/appi.ajp.159.6.941 